\title{
RAMAN SPECTROSCOPY OF MONOSODIUM URATE CRYSTALS: A NOVEL TOOL FOR NON-INVASIVE GOUT DIAGNOSIS
}

\author{
by \\ Faizan Bilwani \\ Bachelors of Mechanical Engineering, Ryerson University, 2014 \\ A thesis \\ presented to Ryerson University \\ in partial fulfillment of the \\ requirements for the degree of \\ Masters of Applied Science \\ in the Program of \\ Mechanical and Industrial Engineering
}

Toronto, Ontario, Canada, 2017

CFaizan Bilwani 2017 


\section{Author's declaration}

\section{AUTHOR'S DECLARATION FOR ELECTRONIC SUBMISSION OF A THESIS}

I hereby declare that I am the sole author of this thesis. This is a true copy of the thesis, including any required final revisions, as accepted by my examiners.

I authorize Ryerson University to lend this thesis to other institutions or individuals for the purpose of scholarly research

I further authorize Ryerson University to reproduce this thesis by photocopying or by other means, in total or in part, at the request of other institutions or individuals for the purpose of scholarly research.

I understand that my thesis may be made electronically available to the public. 


\title{
Abstract
}

\author{
Raman spectroscopy of Monosodium Urate crystals: A novel tool for non-invasive gout diagnosis \\ Master of Applied Science, 2017 \\ Faizan Bilwani \\ Department of Mechanical \& Industrial Engineering \\ Ryerson University
}

Gout is the most common form of inflammatory arthritis. It results in the deposition of monosodium urate crystals (MSU) at the surface of a joint or the articular cartilage. The gold standard for gout diagnosis is synovial fluid (SF) analysis which requires aspiration of the fluid and subsequent analysis by polarized light microscopy (PLM). This has poor reproducibility, is invasive and requires trained personal to perform the analysis. Raman spectroscopy (RS) has the potential to be a non-invasive diagnostic tool that can detect the presence of MSU crystals. The purpose of the research was to determine whether Raman spectroscopy applied onto the surface of a joint could detect MSU crystals through the skin. Two clinical studies were conducted, entitled the Pilot study (Pilot) and the sons of gout study (SOG). Pilot $(n=20)$ considered ten clinically diagnosed gout sufferers and ten clinically diagnosed Osteoarthritis (OA) patients that acted as control. SOG $(\mathrm{n}=25)$ considered nine asymptomatic patients where gout was confirmed by clinicians at time of experiment by ultrasound, and 16 Non-Gouty patients, which did not show any signs of gout. An algorithm was implemented in Matlab ${ }^{\circledR} 2016$ that removed background florescence, performed denoising and identified the presence or absence of MSU peaks. The comparisons were made against Raman peaks that are known to relate to MSU according to the literature. Three peak combinations, entitled C1, C2 and $\mathrm{C} 3$ were evaluated based on their resulting sensitivities and specificities for both studies. $\mathrm{C} 1$ was chosen as it provided the highest sensitivity for both studies. Pilot was found to have a sensitivity and specificity of 0.8 and 0.7 , respectively. SOG had a sensitivity and specificity of 1 and 0.5 , respectively. The results indicated that RS diagnosis is able to achieve good to high sensitivity comparable to other gout detection techniques but a moderate to good specificity. The results also show that RS is fully capable of detecting MSU crystals in-vivo, but results in a high number of false positives, 2 for Pilot and 7 for SOG. However, the false positives in Pilot may be attributed to the control subjects suffering from osteoarthritis, which can be a precursor of gout, and the false positives in the SOG study may be a result of ultrasound (US) being used as the confirmatory diagnostic technique that RS is being compared to. US is known to have sensitivity as low as 0.22 and is operator-dependent. Larger population studies are needed to confirm the ability of RS as a diagnostic tool for detecting gout. 


\section{Acknowledgements}

I would like to thank my supervisor Dr. Mark Towler for his continued support and patience, as well as his invaluable advice, help and expertise in completing this thesis. I would like to thank the personnel involved at the Academic Rheumatology, University of Nottingham, Nottingham, UK for recruiting subjects and acquiring data for this thesis. I also wish to thank Dr.Abhishek Abhishek for taking on these studies with us.

I would also like to thank Dr. Declan Curran for advice, support and assistance in interpreting the data in the thesis. I also wish to thank my colleagues and friends of the CMD group for their support, quite relaxing coffee breaks and extremely one-sided yet cathartic Squash sessions. Last but certainly not least; I would like to thank my family and wife Maryam for their continued moral support, love and encouragement throughout my life and especially in the past two years. 


\section{Table of contents}

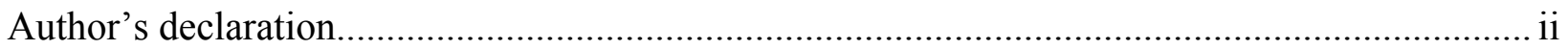

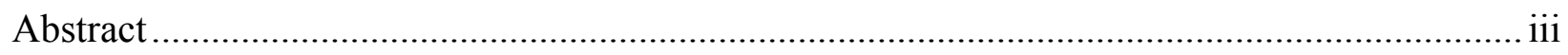

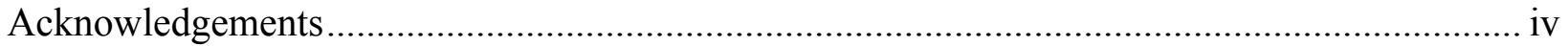

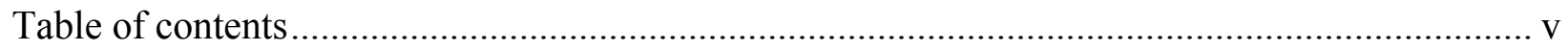

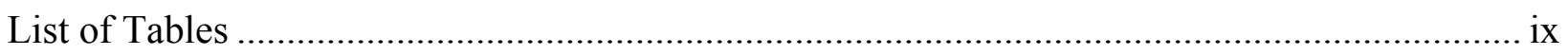

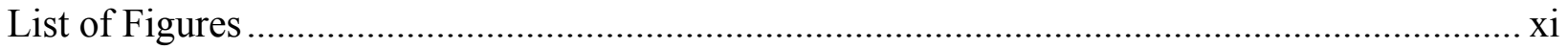

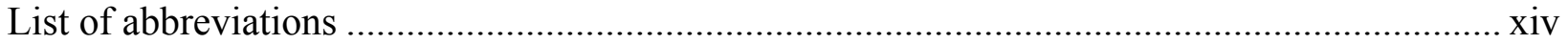

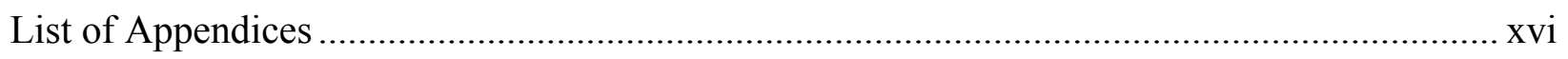

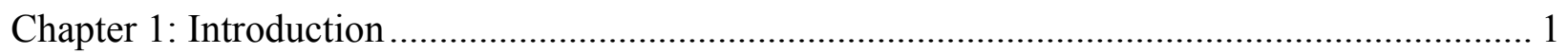

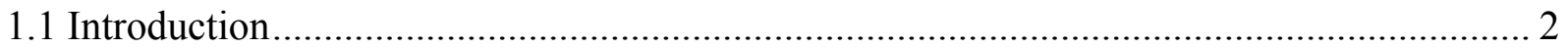

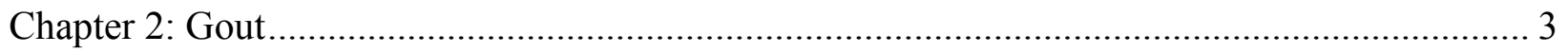

2.1 Definition of the disease ........................................................................................ 4

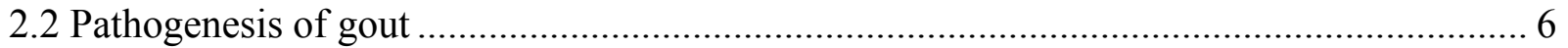

2.2.1 Uric acid in Humans.........................................................................................................

2.2.2 Serum Urate levels and crystal formation .......................................................................

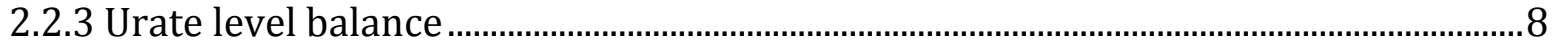

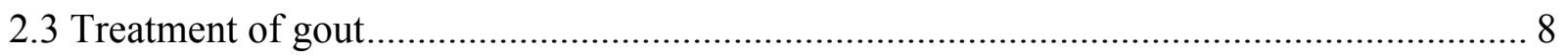

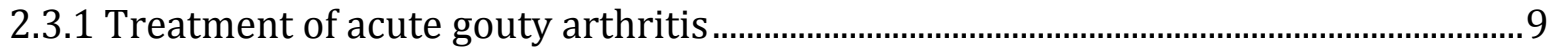

2.3.2 Treatment of Chronic gouty arthritis and Hyperuricaemia ........................................ 9

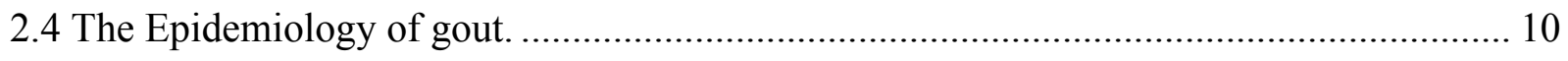

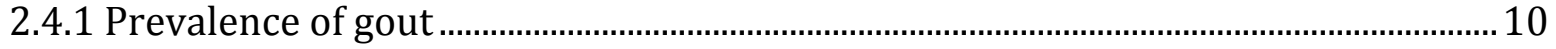

2.4.2 Risk factors and comorbidities of gout............................................................................ 11

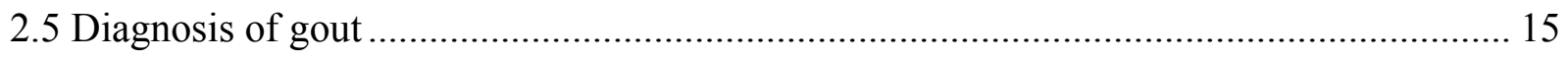

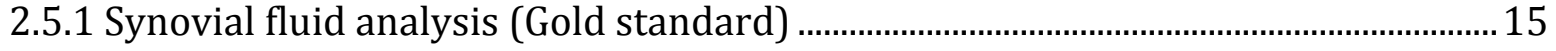

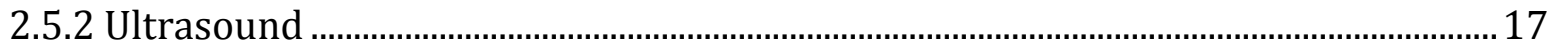

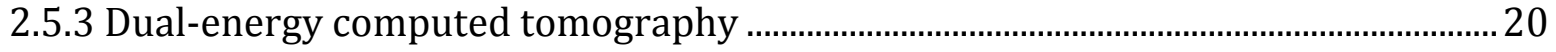

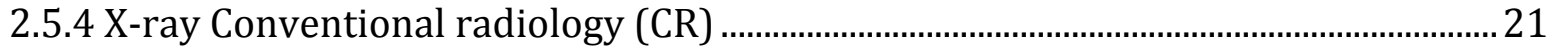

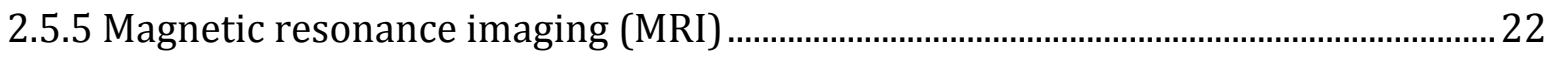


2.6 The need for a non-invasive diagnostic tool for gout detection................................... 23

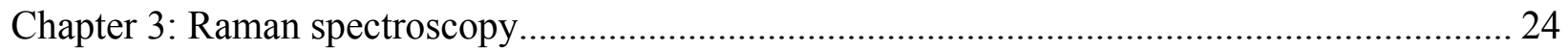

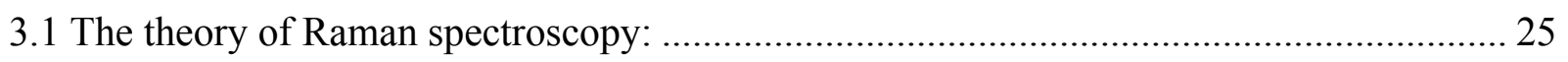

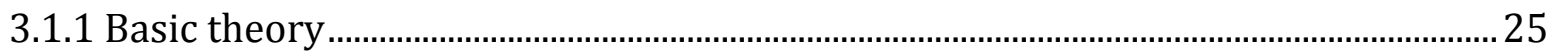

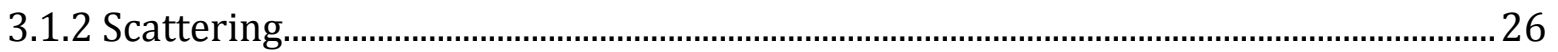

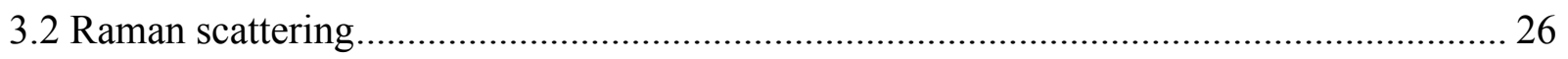

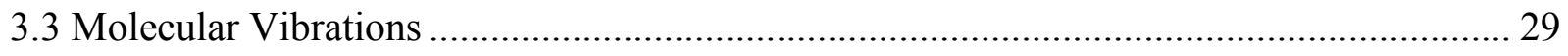

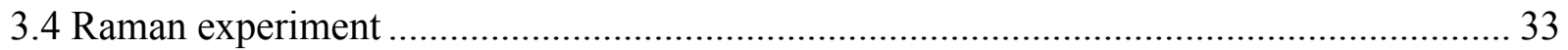

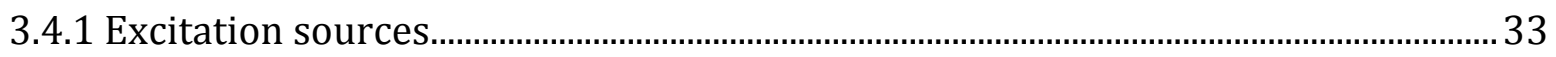

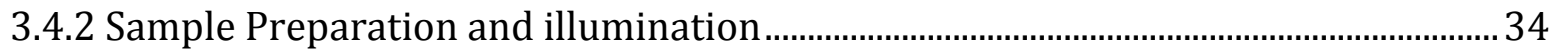

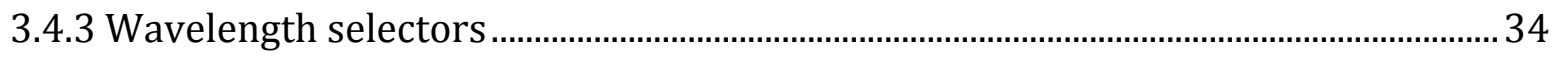

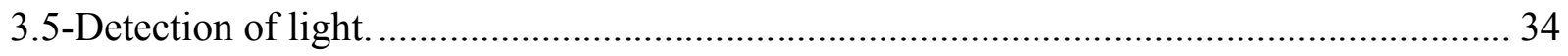

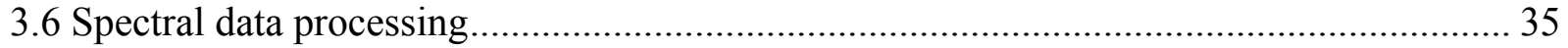

3.7 Spatially-offset Raman spectroscopy (SORS) …..................................................... 35

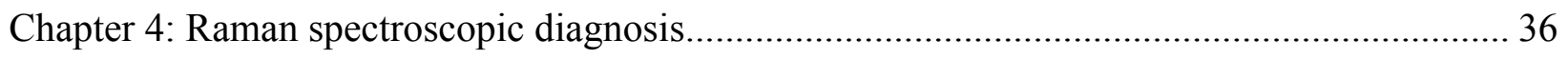

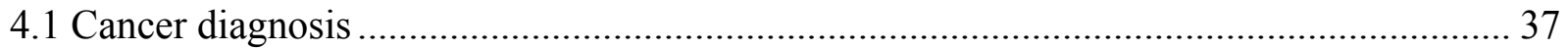

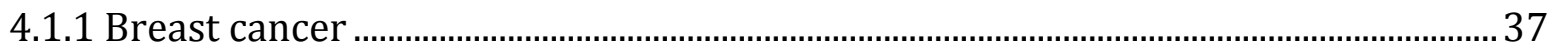

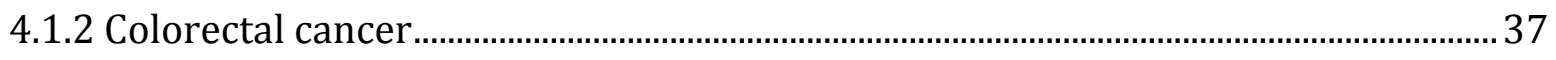

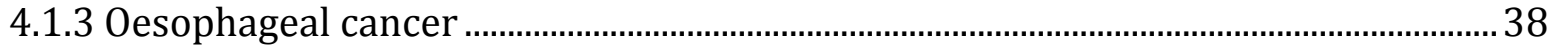

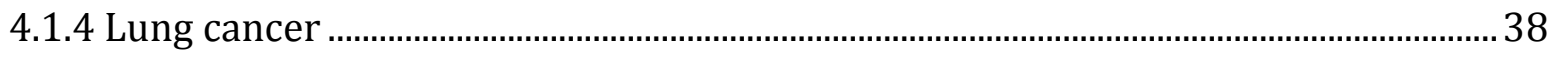

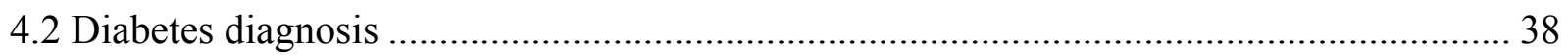

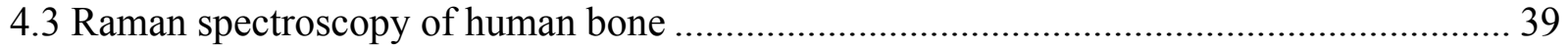

4.4 Raman spectroscopy for diagnosing Osteoporosis and screening for fracture risk ........... 40

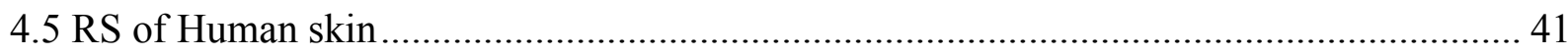

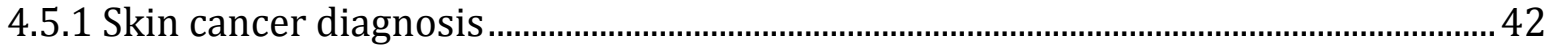

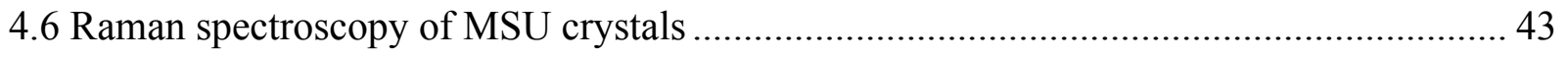

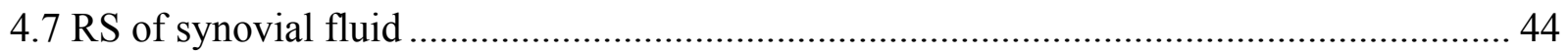

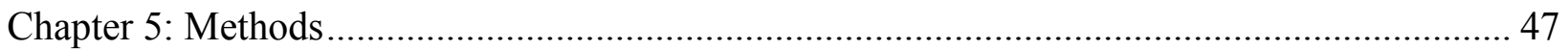

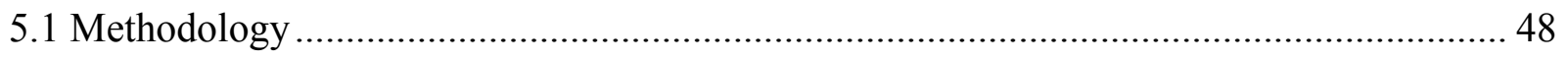

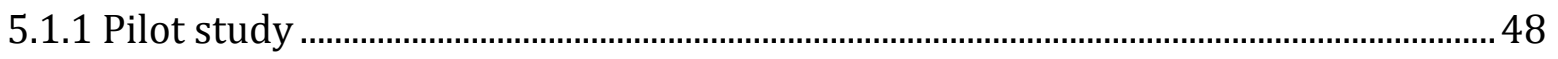

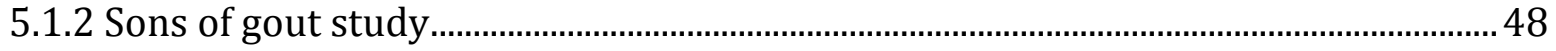




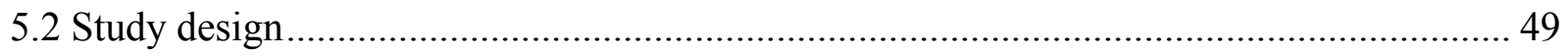

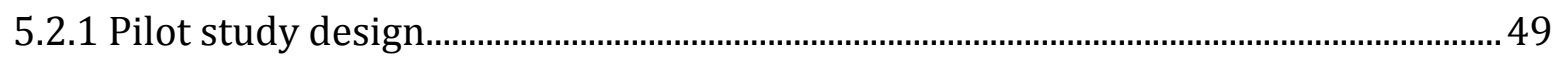

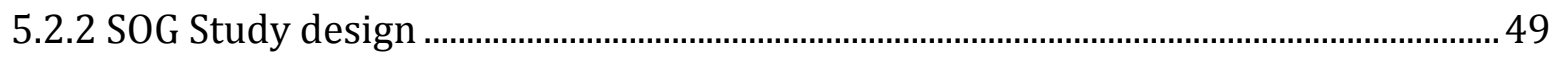

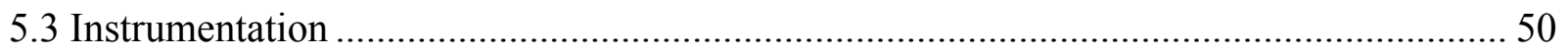

5.3.1 Snowy range Sierra model for pilot study ………………………………………........ 50

5.3.2 Snowy Range Fibre probe model for the SOG study ................................................... 51

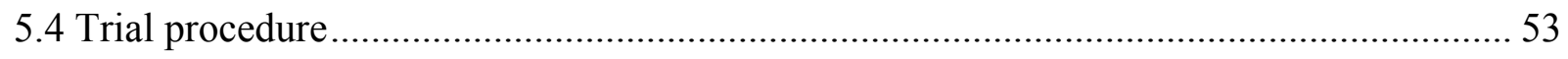

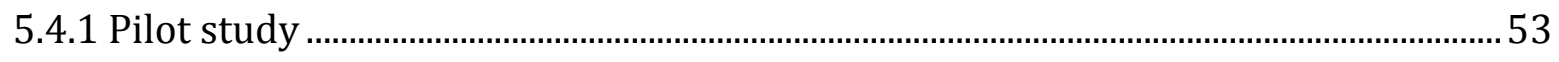

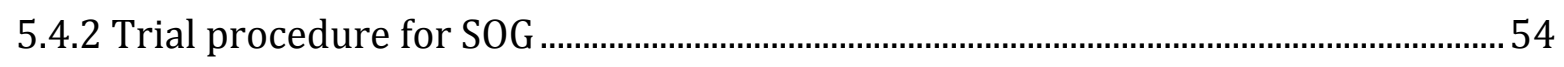

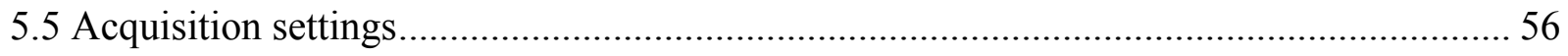

5.5.1 Acquisition settings for Pilot................................................................................

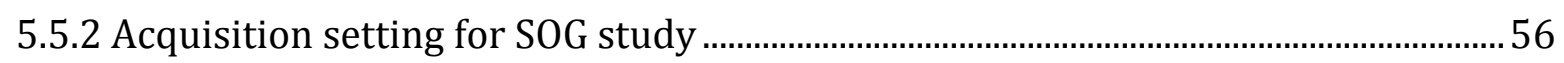

5.7 Matlab routine for Determining MSU presence Automatically (both studies).................. 57

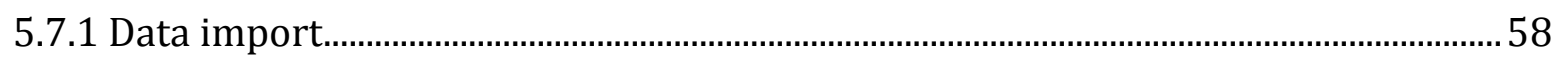

5.7.2 Background florescence removal .............................................................................. 59

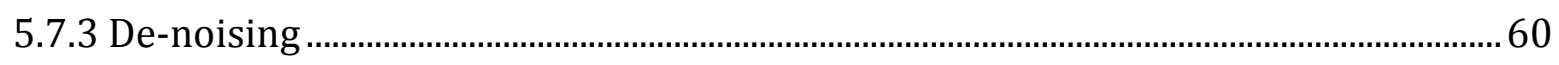

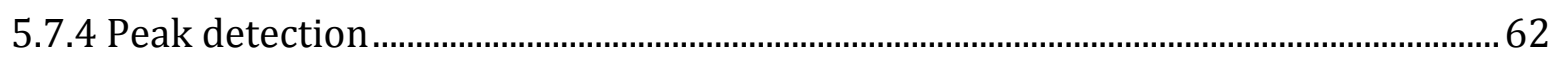

5.7.5 Peak comparison...................................................................................................... 62

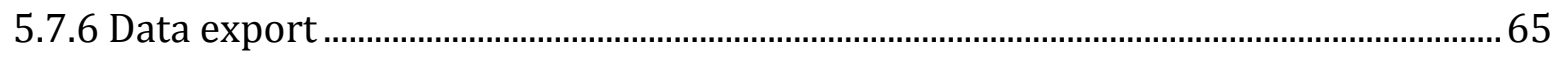

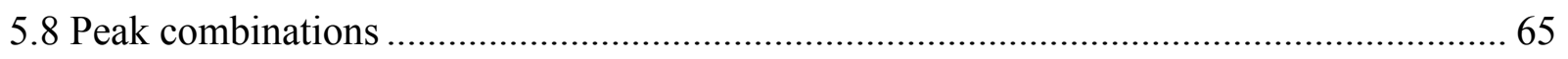

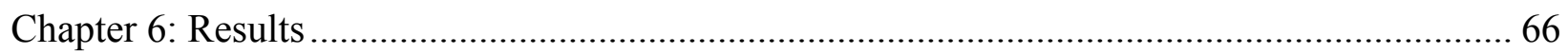

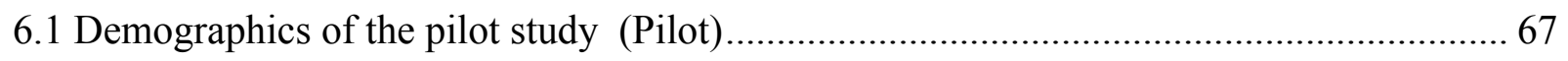

6.1.1 Gout Subjects SUA levels for pilot study …………………………………………........ 68

6.1.2 OA subjects SUA levels for pilot study ……………........................................................ 68

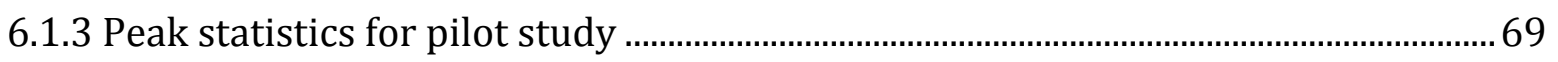

6.1.4 Raman scan examples for Pilot...................................................................................... 71

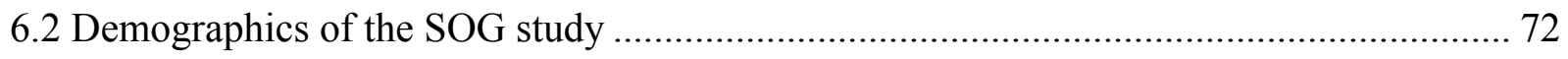

6.2.2 Ultrasound features ....................................................................................................

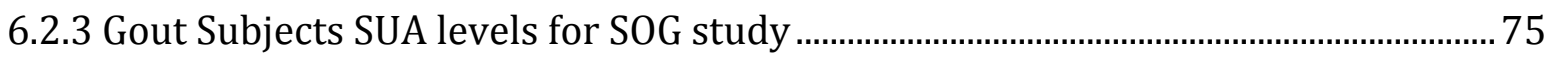

6.2.4 Non-gouty Subjects SUA levels for SOG study ……………………………………..... 75

6.2.5 Peak statistics for SOG study.................................................................................... 
6.2.6 Raman scan examples for SOG study ………………………………………………... 78

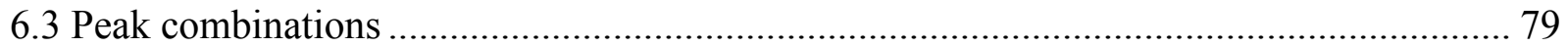

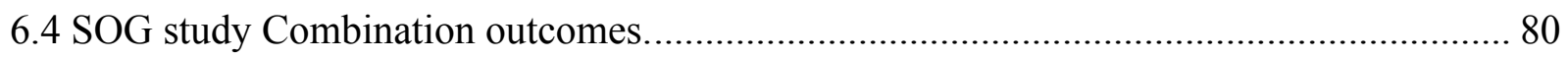

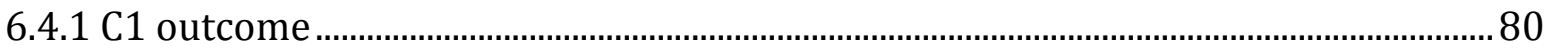

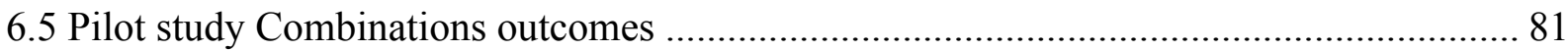

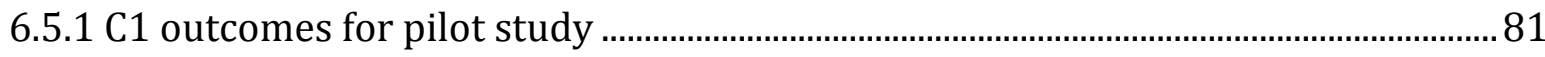

6.5.2 C2 outcomes for pilot study …………………………………………………………... 82

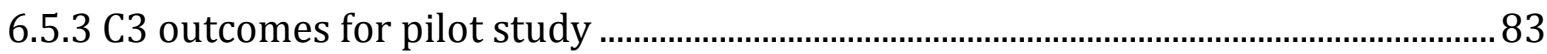

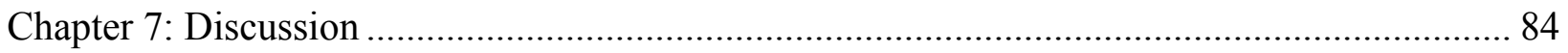

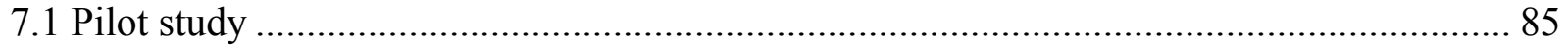

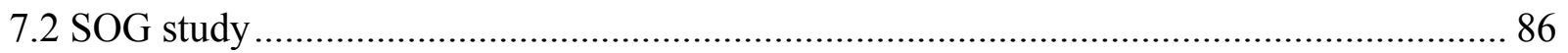

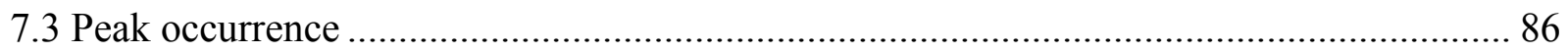

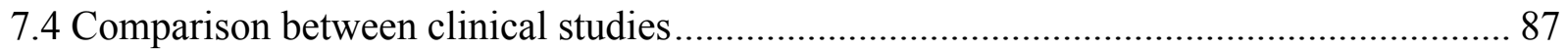

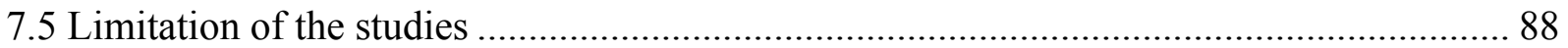

7.6 Comparison of Diagnostic results against existing techniques.......................................... 90

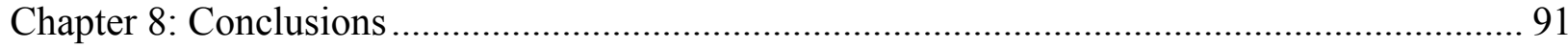

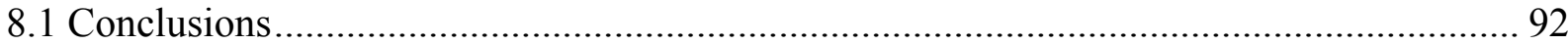

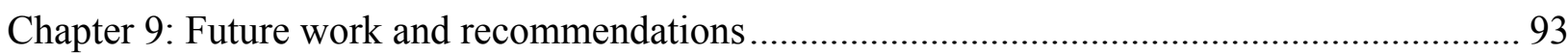

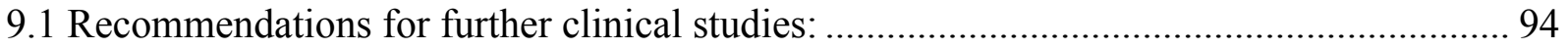

9.2 Recommendations for Instrumentation and post processing: ............................................. 94

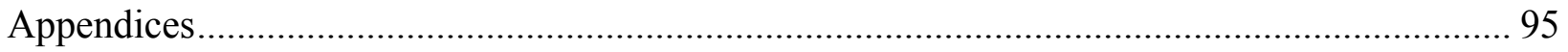

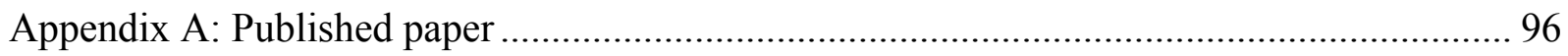

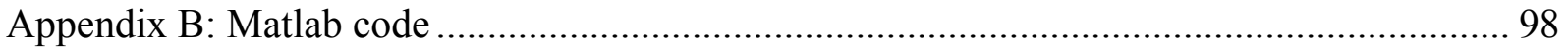

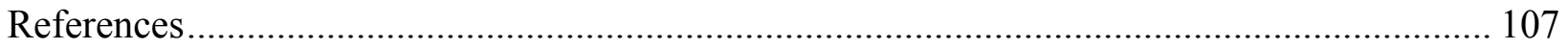




\section{List of Tables}

Table 1. Outline of classic stages of gout with brief stage descriptions................................. 4

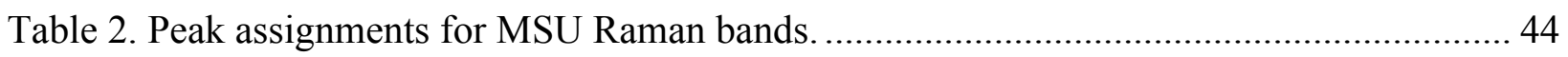

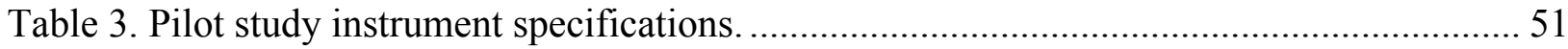

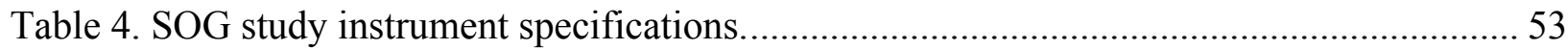

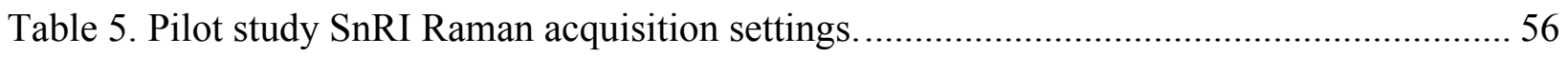

Table 6. SnRI Fibre probe Raman acquisition settings for SOG study. ................................. 56

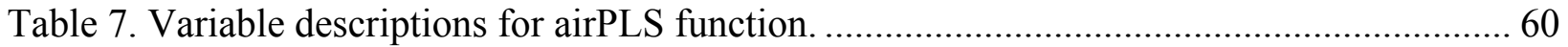

Table 8. Variable descriptions for wavelet_denosing function. ............................................ 61

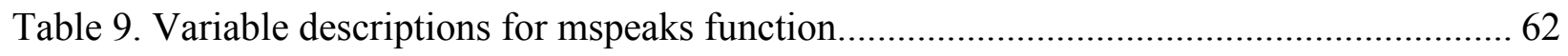

Table 10. Variable descriptions for peak comparison function. ............................................ 63

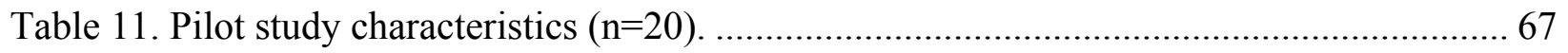

Table 12. SUA levels and Average Raman peaks detected per scan for gout subjects. .............. 68

Table 13. SUA levels and Average Raman peaks detected per scan for OA subjects. ................ 69

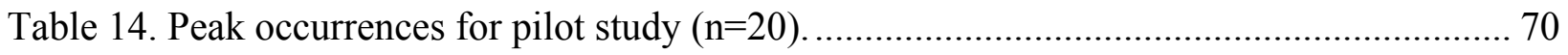

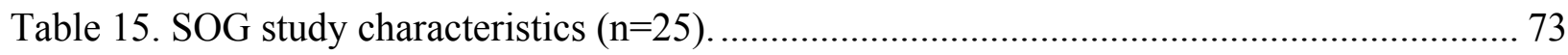

Table 16. Ultrasound Features observed for SOG study $(n=25)$ for both left $(L)$ and right $(\mathrm{R})$

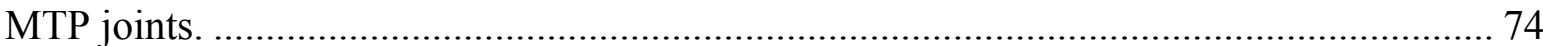

Table 17. SUA levels and Average Raman peaks detected per scan for gout subjects from the

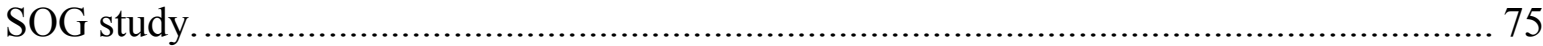

Table 18. SUA levels and Average Raman peaks detected per scan for Non-gouty subjects from

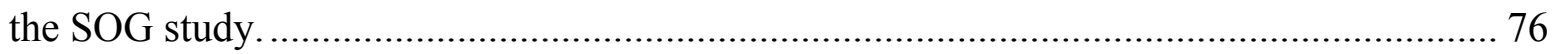

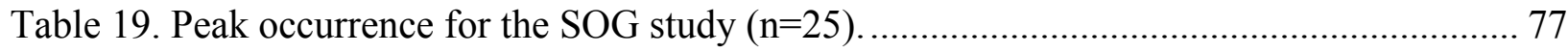

Table 20. Peak combinations for Raman positive diagnosis. ................................................... 80

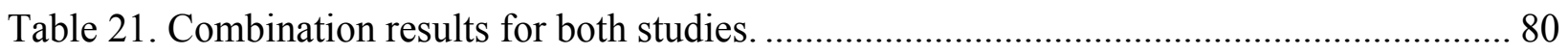

Table 22. Diagnostic results for SOG study combinations.................................................... 81

Table 23. diagnostic statistics for SOG study combinations. .............................................. 81

Table 24. Diagnostic results for pilot study for combination $\mathrm{C} 1$.......................................... 82

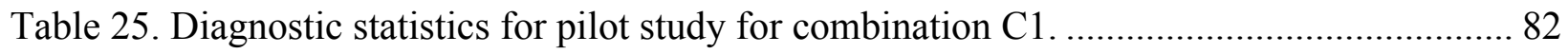


Table 26. Diagnostic results for pilot study for combination C2 …................................... 82

Table 27. Diagnostic statistics for pilot study for combination C2 .................................... 83

Table 28. Diagnostic results for pilot study for combination C3 ....................................... 83

Table 29. Diagnostic statistics for pilot study for combination $\mathrm{C} 3$. ........................................ 83

Table 30. Comparison of RS with existing Diagnostic techniques for gout............................. 90 


\section{List of Figures}

Figure 1. Image of a patients' hand with Tophaceous gout [7] ................................................. 5

Figure 2. The three stages of gout disease and increase in intensity of pain [7] .......................... 5

Figure 3. Relationship between Serum Urate level and incidence of gout ..................................... 6

Figure 4. Crystal formation and crystal-induced inflammation process........................................ 7

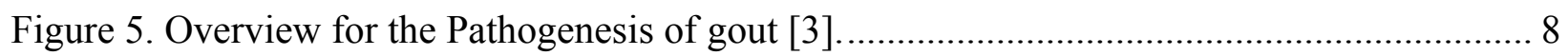

Figure 6. Relation between SUA levels and prevalence of gout in 4257 subjects in France.

Produced from data given in [23] .............................................................................. 11

Figure 7. Age and gender specific gout incidence trend of UK in 2012 [20]............................ 12

Figure 8. Age specific prevalence of gout in the UK for Men and Women [18] ....................... 13

Figure 9. Harvard healthy eating pyramid for dietary influences on gout................................... 14

Figure 10. Needle asipiration procedure for the first MTP joint [26] ........................................ 15

Figure 11. PLM image of MSU crystals, which are intracellular and needle-shaped objects coloured blue (arrow) and yellow (triangle) [26] ............................................................. 17

Figure 12. Ultrasound image of DCS feature labelled as metatarsal head [33]........................... 18

Figure 13. Ultrasound image of HED feature [35] ............................................................... 19

Figure 14. Ultrasound image of effusion feature (thin arrows) [35] ........................................... 19

Figure 15. DECT images of MSU crystal deposits, which are coloured red. A knee and D foot

[39]

Figure 16. X-Ray image of gouty fingers with tophi on the index finger [34] ............................ 21

Figure 17. T2 weighted MRI knee scan with a tophi feature (wide arrow) [41] ......................... 22

Figure 18. Electromagnetic spectrum and their associated parameters [46] ............................... 26

Figure 19. Illustration of the three different types of scattering [45].......................................... 27

Figure 20. Energy levels for the three different types of scattering [50].................................... 28

Figure 21. Raman spectrum of CCL4 with both Stokes and anti-Stokes scattering [46] ............. 29

Figure 22. Visual quantification of polarizability of a $\mathrm{CO} 2$ molecule [45]................................. 30

Figure 23. CH3 methyl group stretching vibrations [45]........................................................... 31

Figure 24. CH3 methyl group bending vibrations [45] ........................................................ 32

Figure 25. In plane and out plane bending vibrations of $\mathrm{CH} 2$ of the methylene group [45] ........ 32 
Figure 26. RS scan for whole human bone, obtained using a Fourier-transform Raman

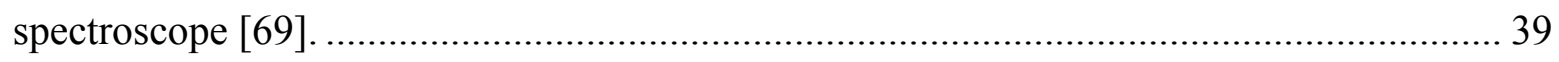

Figure 27. RS scan of a human nail for a spectral range of $300 \mathrm{~cm}-1$ to $1800 \mathrm{~cm}-1$ [74]........... 41

Figure 28. Custom -built inverted Raman microspectrometer housing for in-vivo Raman

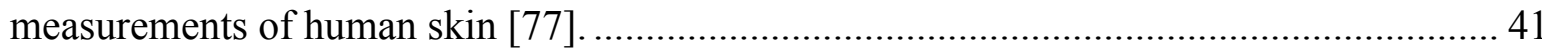

Figure 29. in-vitro Raman spectroscopy results for different layers of human skin, A is stratum corneum, B is epidermis, $\mathrm{C}$ is dermis and $\mathrm{D}$ is human collagen I [77] ............................ 42

Figure 30. Raman spectrum of synthetic monosodium urate [43] ...................................... 43

Figure 31. Chemical structure of monosodium urate (C5H3N4O3.Na) [43] .......................... 43

Figure 32. RS scan of synovial fluid after digestion and centrifugation for normal, gout and

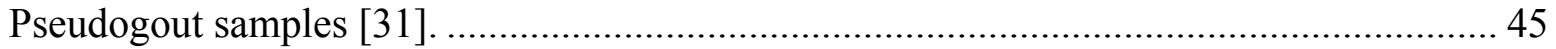

Figure 33. Top-view of SnRI-Sierra models Raman spectroscope used for the pilot study data

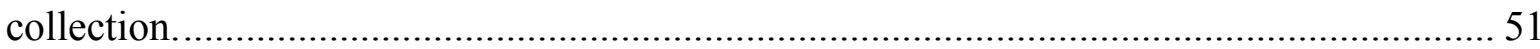

Figure 34. SnRI-Sierra Fiber probe model Raman spectroscope for SOG study data collection. 52 Figure 35. SOG-probe focal length adjusted to standoff position one to acquire RS scans for SOG study. 52

Figure 36. Trial setup for the pilot study, showing the Raman spectroscope positioned at the

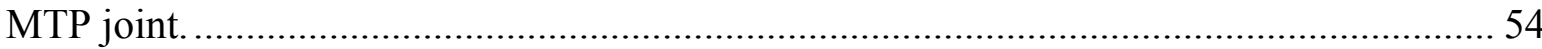

Figure 37. SOG study scanning positions: (a) Position 1, (b) Position 2 and (c) Position 3....... 55

Figure 38 Raman processing algorithm for spectral processing for both studies ..................... 57

Figure 39. Simulated MSU with labelled Raman peak wavenumbers, recreated from [43]....... 58

Figure 40. Background removal demo, containing the simulated MSU, corrected MSU and baseline fit plots. 59

Figure 41. De-noising demo, containing the simulated signal with noise, without noise and the de-noised signal.

Figure 42. Simulated MSU with labelled Raman peak wavenumbers, recreated from [43] ....... 70

Figure 43. gouty subject example of de-noised Raman scan with MSU trace overlaid.............. 71 Figure 44. Non-gouty (OA) subject example of de-noised Raman scan with MSU trace overlaid.

Figure 45. gouty vs Non-gouty (OA) subject example with their respective de-noised Raman scans. 
Figure 46. SUA level distribution for SOG study, gouty and Non-gouty populations................ 73

Figure 47. Gouty subject example of a denoised Raman scan with MSU trace overlaid............ 78

Figure 48. Non-gouty subject example of denoised Raman scan with MSU trace overlaid. ....... 78

Figure 49. Non-gouty vs gouty example subject with their respective denoised Raman scans. .. 79 


\section{List of abbreviations}

\begin{tabular}{|c|c|}
\hline ACR & American college of rheumatology \\
\hline BQT & bone quality test \\
\hline CPPD & Calcium pyrophosphate dihydrate crystal \\
\hline $\mathrm{CW}$ & Continuous wave \\
\hline $\mathrm{CR}$ & X-ray Conventional radiology \\
\hline DCS & Double contour signal \\
\hline DECT & Dual-energy computed tomography \\
\hline DOF & degrees of freedom \\
\hline DXA & Dual-energy X-ray absorptiometry \\
\hline EW & electromagnetic wave \\
\hline FTIR & Fourier-transform Raman spectroscopy \\
\hline FWHH & Full-width at half height \\
\hline FDR & False discovery rate \\
\hline FOR & False Omission rate \\
\hline HEDs & Hyperechoic depositions \\
\hline LDA & linear discriminant analysis \\
\hline MSU & monosodium urate crystals \\
\hline MTP & first metatarsophalangeal \\
\hline MRI & Magnetic resonance imaging \\
\hline NPV & Negative predictive value \\
\hline NSAIDs & Non-steroidal Anti-inflammatory drugs \\
\hline OA & Osteoarthritis \\
\hline PPV & Positive predictive value \\
\hline Pilot & Pilot study \\
\hline PCA & principal component analysis \\
\hline PLM & Polarized light microscopy \\
\hline RS & Raman spectroscopy \\
\hline SF & synovial fluid \\
\hline SUA & Serum Uric Acid \\
\hline SORS & Spatially-offset Raman spectroscopy \\
\hline SOG & sons of gout study \\
\hline SnRI & Snowy Range Instruments \\
\hline SLN & sentinel lymph node \\
\hline US & Ultrasound \\
\hline
\end{tabular}


UV

ULT ultraviolet

Urate-lowering therapy 


\section{List of Appendices}

Appendix A: Published paper.................................................. 96

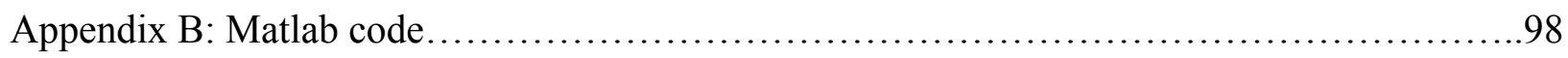




\section{Chapter 1: Introduction}




\subsection{Introduction}

Gout is the most common form of inflammatory arthritis in men aged over 40y and has prevalence of over 3\% in Europe and North America [1]. The risk of developing gout increases with age for both men and women but is more common in males. Gout occurs due to the deposition of Monosodium Urate crystals (MSU) in joint fluid or on the surface of articular cartilage. This deposition tends to occur predominantly on the first metatarsophalangeal (MTP) joint [2]. Deposition of such crystals is promoted due to a biological abnormality known as hyperuricemia, a state that occurs when there is an elevated concentration of urate (the ionized form of uric acid) in the blood and when Serum Uric Acid (SUA) surpasses the solubility threshold of $6.8 \mathrm{mg} / \mathrm{dL}$ in vitro [2]. The clinical significance of gout and hyperuricemia lies in its associations with diseases such as nephropathy, insulin resistance syndrome, and hypertension $[3]$.

The gold standard for diagnosing gout is analysis of aspirated synovial fluid (SF) using polarized light microscopy [4]. However, SF analysis has poor reproducibility, is operator dependent, invasive, dependent on the crystal concentration and cannot currently be automated $[5]$.

The objective of this work was to evaluate the ability of Raman spectroscopy (RS) to detect MSU crystals without invasion to skin. This objective was tested using two separate clinical studies and the results analyzed using Matlab® to detect known MSU peaks, determine a criterion and evaluate its performance against an accepted reference standard for MSU. 
Chapter 2: Gout 


\subsection{Definition of the disease}

Gout is a disorder in which the deposition of Monosodium Urate Crystals (MSU) occurs in joint fluid or on the surface of articular cartilage [2]. Deposition of such crystals occurs due to a biological abnormality known as hyperuricemia. Urate is an end-product of purine metabolism [6]. Food sources of purine include red meat, beer, seafood and certain vegetables [6]. The concentration of uric acid in the blood stream is determined using a Serum Uric Acid (SUA) blood test. An indicator for gout is when SUA surpasses the solubility threshold of $6.8 \mathrm{mg} / \mathrm{dL}$ in vitro [2]. This is the most important risk factor for the development of gout [5]. These inflammatory reactions tend to occur due to soft tissue depositions of MSU crystals [2]. There are three stages of classic gout and these are reported in Table 1.

Table 1. Outline of classic stages of gout with brief stage descriptions.

Stage 1: Asymptomatic Hyperuricemia

- Solubility threshold of $6.8 \mathrm{mg} / \mathrm{dL}$ has been crossed

- $\quad$ No symptoms associated with Hyperuricemia occur, such as Gouty arthritis or Tophi [2], [7]

Stage 2: Acute intermittent gout

- Occurs after decades of Asymtomatic Hyperuricemia

- Recurrent attacks of articular and periarticular inflammation at one or more joints

- Initially, attacks are not frequent with intervals that can span over years

- The duration of attacks ranges from few hours to 2 weeks.

- As the stage progresses, attacks become more frequent, they last longer and affect more joints.

Stage 3: Advanced gout (also referred to as Chronic Tophaceous gout)

- Usually known to develop 10 or more years after entering stage 2

- For certain patients it tends to be the first clinical manifestation of gout.

- Appear as firm swellings [5]

- Can ulcerate as a white chalky material

- A larger structure formed from accumulated MSU crystals is shown in Figure 1 


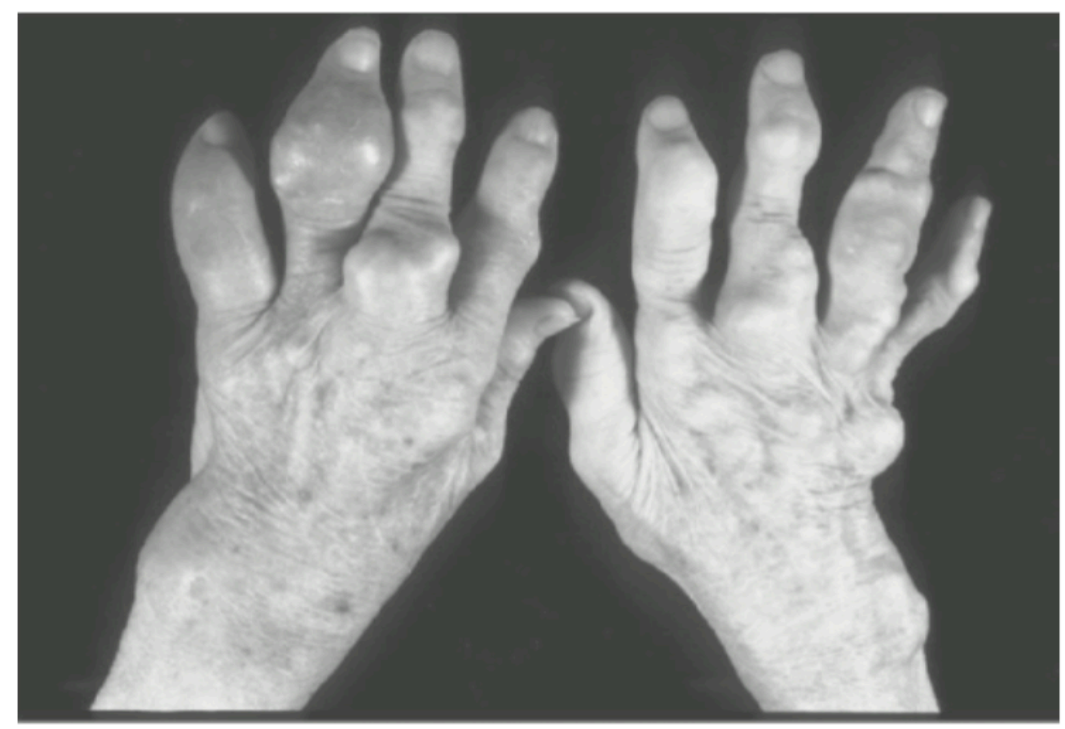

Figure 1. Image of a patients' hand with Tophaceous gout [7].

The clinical significance of both gout and hyperuricemia lie in their associations with diseases such as nephropathy, obesity, and hypertension [3]. Hypertension is a cardiovascular disease that results in high-blood pressure [8] and nephropathy is form of renal disease, occurring due to uric acid urolithiasas [2]. As shown in Figure 2, the intensity of pain increases with each stage of gout; the final stage being the most intense.

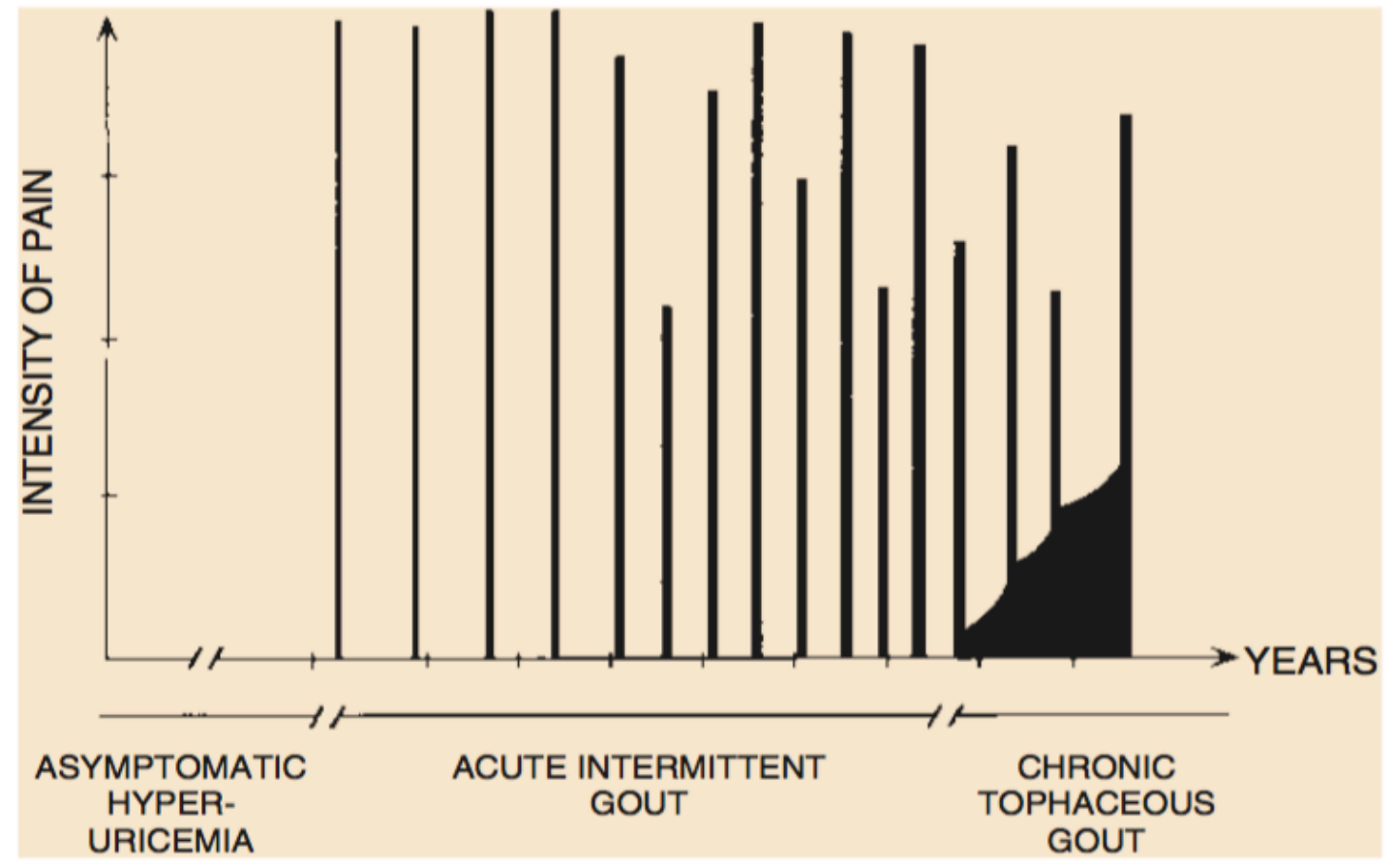

Figure 2. The three stages of gout disease and increase in intensity of pain [7]. 


\subsection{Pathogenesis of gout}

The onset of gout is well understood [2], [3], [9]. This chapter will focus on key stages and factors in the development of gout.

\subsubsection{Uric acid in Humans}

Humans and great apes are the only mammals that develop gout [3]. In most animals (amphibians, fish and non-primate mammals), uric acid produced from purine metabolism tends to go through oxidative degradation by the uricase enzyme that essentially breaks down uric acid and produces the more soluble compound allantoin [2], [3]. This enzyme is missing in humans and great apes, who lost the ability to process uric acid over 5 million years ago, due to mutations that rendered the uricase gene non-functional [10]. Its absence results in human plasma urate levels that are approximately 10 times higher than in most other mammals.

\subsubsection{Serum Urate levels and crystal formation}

Urate is an ionized form of Uric acid, which is a weak acid with $\mathrm{pKa}$ of 5.8 [3]. The concentration of urate in physiological fluids is known to have a positive relationship with the risk of super saturation and crystal formation [3]. Increased urate levels correlate positively with the risk of developing gout [11], [12] as shown in Figure 3.

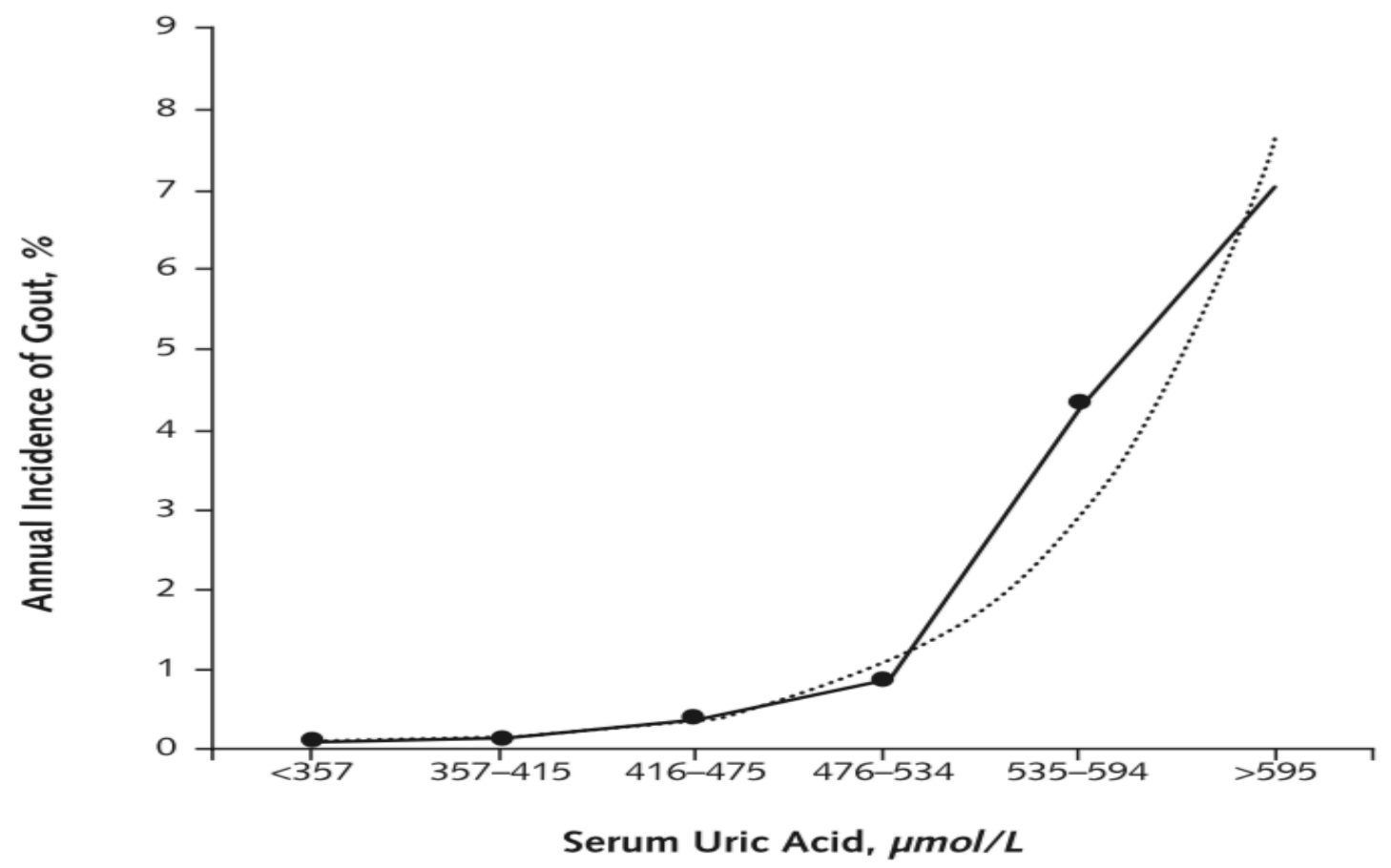

Figure 3. Relationship between Serum Urate level and incidence of gout. 
There are several other factors that influence urate solubility in joints, such as temperature, $\mathrm{pH}$, concentration of cations, articular dehydration levels and nucleating agents [3]. Nucleating agents factor in after MSU crystallizes and cause growth of crystals, whereas the remaining factors affect the process before crystal formation, as seen in Figure 4. The variation in these factors can influence the risk of developing gout. It has been reported [13] that temperature variation has a significant effect on the solubility threshold of the urate ion, where the threshold reduces as the temperature decreases. Therefore, extracellular fluids in joints can have a lower permissible concentration of urate ion if the temperature reduces, essentially increasing the risk of developing gout. This is accepted as the reason why joints such as the MTP are most likely to have MSU crystal deposition, since the MTP joint has a temperature lower than average body temperature [2], [3], [14].

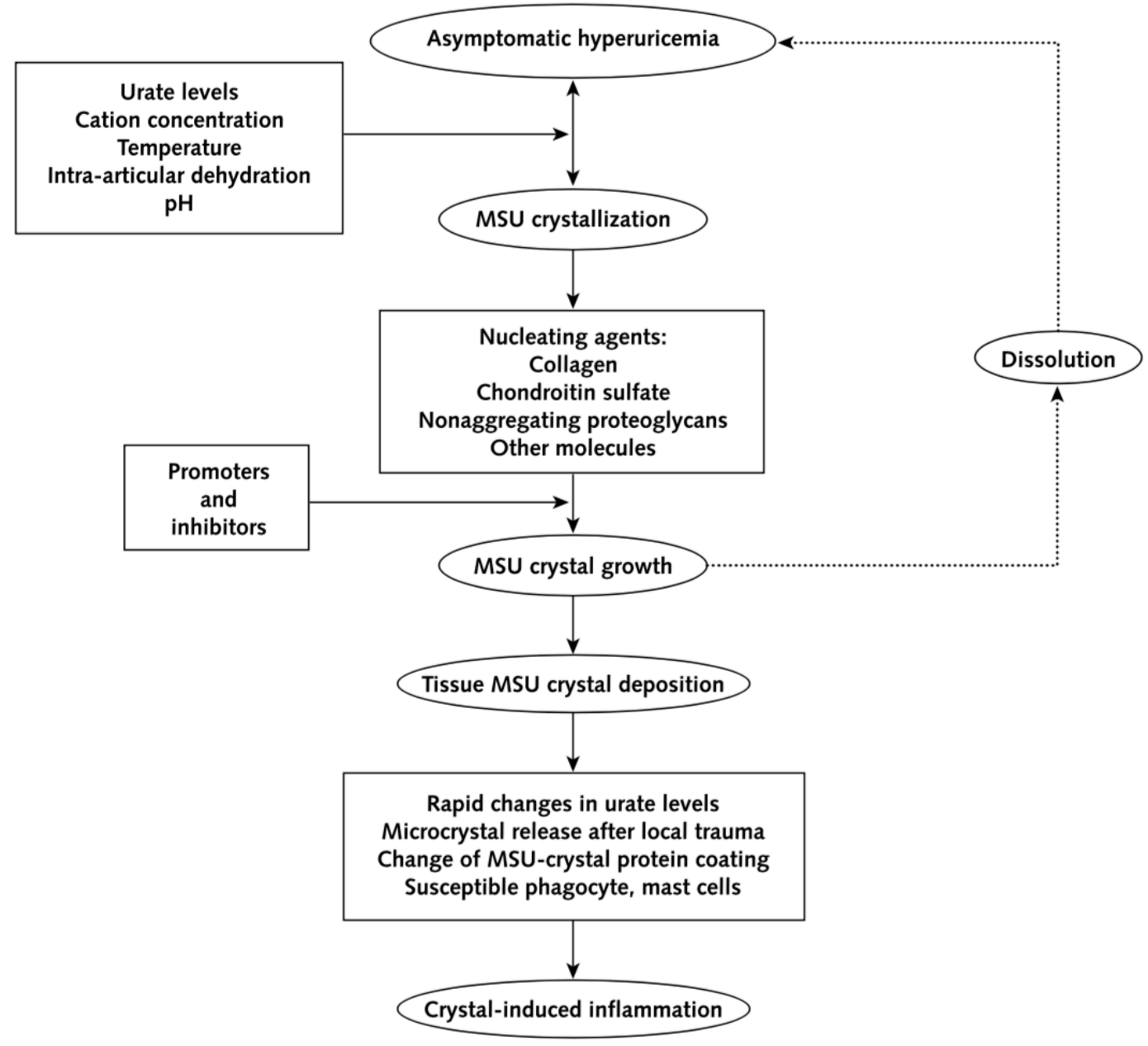

Figure 4. Crystal formation and crystal-induced inflammation process. 


\subsubsection{Urate level balance}

The level of urate is dependent upon diet, endogenous synthesis of purine and rate of excretion [3]. As shown in Figure 5, the amount of purine that enters through diet and the internal synthesis that occurs from within the body, are two precursors to urate production. Hyperuricemia results either by Urate overproduction, under-excretion, or a combination of both.

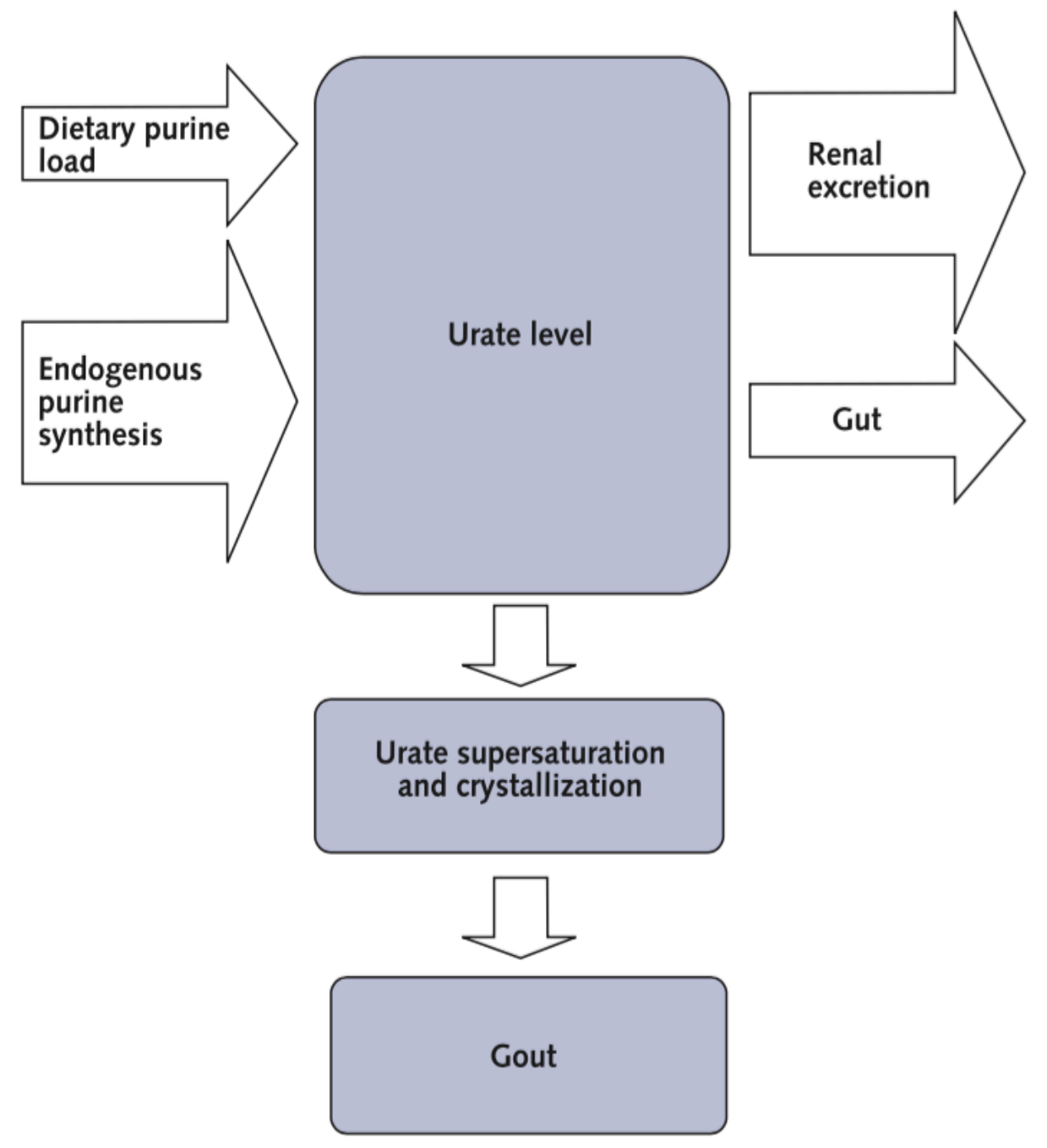

Figure 5. Overview for the Pathogenesis of gout [3].

\subsection{Treatment of gout}

The avoidance of factors that are associated with the development of gout is main approach for its treatment. Generally, there are two points of focus in the treatment of gout, one considers therapies that help reduce inflammation of acute gout, while the other manages hyperuricemia. Overall, there are three stages to managing gout [15]: 
1. Acute attack treatment

a. Patients must not be treated for hyperuricaemia during an acute attack.

2. Lowering excess uric acid

3. Providing prophylaxis to avoid flares

\subsubsection{Treatment of acute gouty arthritis}

Gout diagnosis is confirmed by joint aspiration and synovial fluid analysis to determine whether MSU crystals are present and intracellular, after which a treatment will be prescribed. Non-steroidal Anti-inflammatory drugs (NSAIDs) are the first choice pharmaceutical intervention to treat acute gouty arthritis in the United States [16]. The initial treatment will be conducted with NSAIDs unless the patients exhibits risk factors to contraindicate their use [15]. Age over 65 years and congestive heart failure are among risk factors that prevent the use of NSAIDs. Other options for treatment are [15]:

1) Colchicine

An alkaloid derived from a plant, used to relieve pain in gout sufferers.

2) Corticosteroids,

A steroid hormone used to reduce inflammation administered orally

3) Corticotropin

Promotes adrenal release of corticosteroids for pain relief

4) Intra-articular corticosteroids

Corticosteroids administered through a joint injection

Usually, with the treatment at this stage, success is dependant upon how soon NSAIDs are utilized rather than which specific NSAID is utilized. NSAIDs tend to provide complete treatment of acute gout within 5-8 days of treatment, for $90 \%$ of the subjects [15].

\subsubsection{Treatment of Chronic gouty arthritis and Hyperuricaemia}

Long-standing hyperuricaemia is the reason for uric-acid type kidney stones and chronic tophaceous gout, which is a larger structure formed from MSU crystals, therefore, long-term control of hyperuricaemia is imperative in preventing gouty attacks [15]. Urate-lowering therapy (ULT) is employed to control hyperuricaemia by maintaining the serum urate level (SUA) at 
$<6.0 \mathrm{mg} / \mathrm{dl}$, which is known to resolve tophi. There is not enough evidence to provide a guideline on when to start urate-lowering therapy, but generally ULT is prescribed for patients that have 2 or more attacks a year, although, ULT should not be started during acute attacks [15]. Allopurinol (Dsm Pharmaceuticals Inc, Greenville, United States) is the most commonly prescribed ULT drug; a uricostatic drug, which inhibits xanthine oxidase by interfering with the conversion of hypoxanthine to xanthine that are uric acid precursors. When this drug interferes with this conversion, hypoxanthine does not transform to xanthine, which in turn does not transform to uric acid. Therefore, inhibiting the production of uric acid. The maximum effect of SUA lowering is noticeable within 14 days. Most patients are maintained on a fixed dosage of 100 to $300 \mathrm{mg}$ a day, while patients with renal failure are initially prescribed 50-100 mg a day [15]. Febuxastat (Mylan pharmacueticals Inc, West Virginia, USA) is another ULT drug still under development and not currently favoured over Allopurinol. Probenecid (Mylan pharmacueticals Inc, West Virginia, USA) is a uricosuric drug that is prescribed for patients that have an allopurinol allergy. Recently, Lesinurad (AstraZeneca plc, Cambridge, England) has been used in randomized clinical trials, where it is combined with Xandithin oxidaze inhibitators, such as Allopurinol, to help achieve the target urate level with better efficiency [17].

\subsection{The Epidemiology of gout.}

\subsubsection{Prevalence of gout}

Studies have shown an increase in the prevalence of gout in the past two decades [18]. In 1990 to 1999 , gout prevalence increased from 2.9/1000 people to 5.2/1000 people in the United States. In 2007-2008, gout was reported to affect 3.9\% (8.3 million) of US adults, of which men had a prevalence of 5.9\% (6.1 million) and women 2.0\% (2.2million) [19]. Other studies have found an increase in both instances and severity of gout with more individuals developing tophi [20]. In the UK, gout reportedly increased during the 1970s and 1980s [20], but stabilized between 1990 and 1999 [21]. One study conducted in the UK and Germany between 2000 and 2005 reported a prevalence of $1.3 \%$ for both countries [22]. Most studies tend to show that gout predominantly affects older men, where the overall male to female ratio is approximately 3-4:1 [18]. It is the most common inflammatory arthritis in men aged $>40$ [16]. 


\subsubsection{Risk factors and comorbidities of gout}

\subsubsection{Hyperuricemia}

Chronic hyperuricemia is understood to be a prerequisite to the formation of MSU crystals. The SUA level of the subject is utilized to diagnose and monitor the condition. This level varies between genders, ethnicities and countries [20]. Hyperuricemia is one of the main risk factors in developing gout [18], [20]. Figure 6 shows the prevalence associated with SUA levels determined from a study conducted in France. Although, studies have also suggested that only a minority of subjects with elevated SUA ever tend to develop gout [18], other risk factors such as age, gender and diet are important.

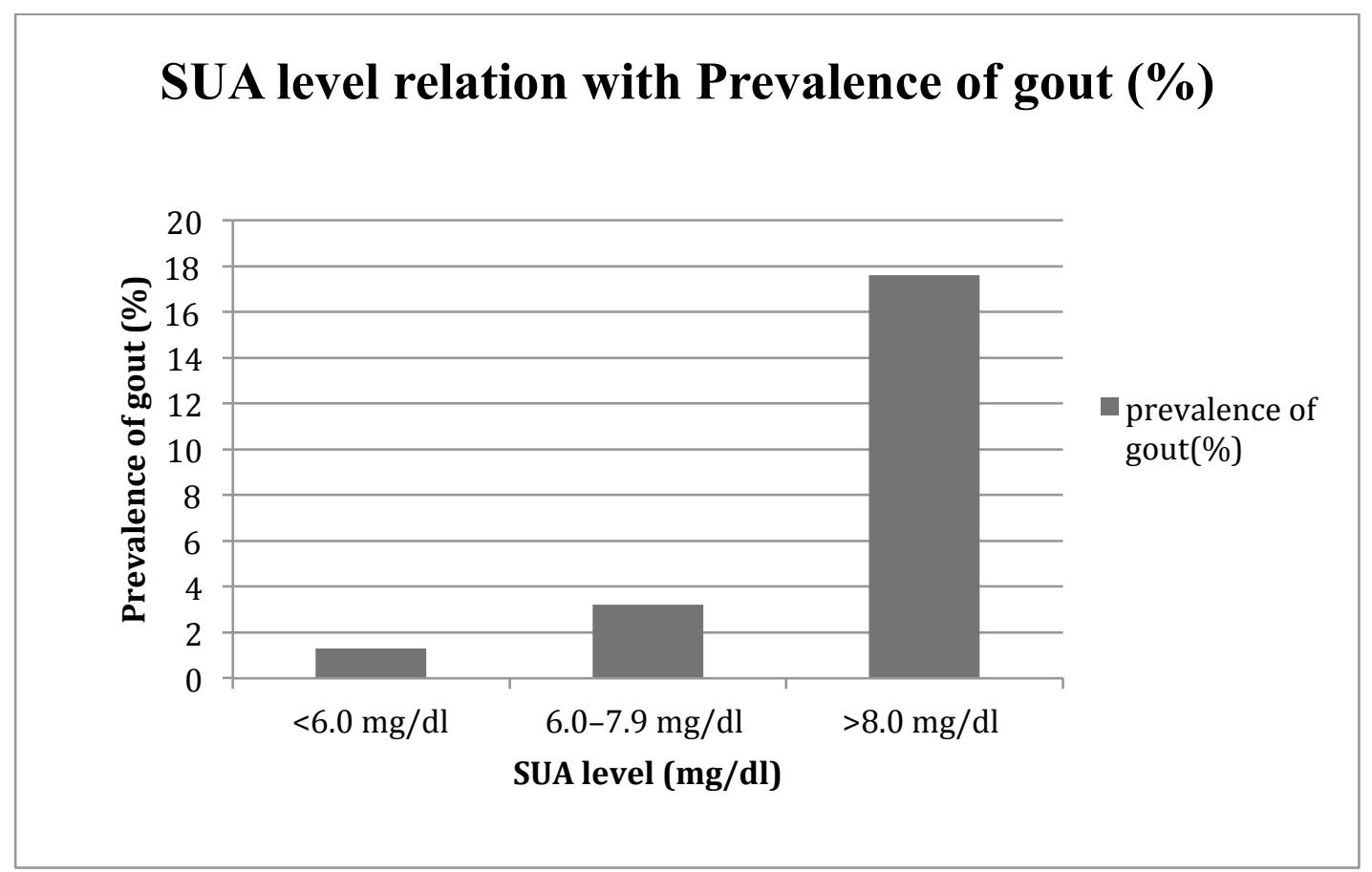

Figure 6. Relation between SUA levels and prevalence of gout in 4257 subjects in France. Produced from data given in [23].

\subsubsection{Genetics}

The contribution of genetics as a risk factor for gout is supported by epidemiological and clinical evidence [20]. There are differences in prevalence by ethnicity and genetics [19], [20] . Evidence is present for familial connections of gout; the disease can be passed down to future generations [24]. In a population study in Taiwan, a $35.1 \%$ heritability in men and $17.0 \%$ in women was reported, which shows the significance of genetics towards the incidence of gout. 
Environmental affects can also alter genetics as a study in New Zealand reported a $900 \%$ increase in risk of gout in Tokelauan migrants against non-migrants [20]. Furthermore, there are certain disorders that lead to a over production of uric acid, from enzyme defects that affect purine metabolism [18].

\subsubsection{Age and Gender}

In men, the risk of gout increases steadily with age, while in women the risk sees a sharp increase after menopause [20] (Figure 7).

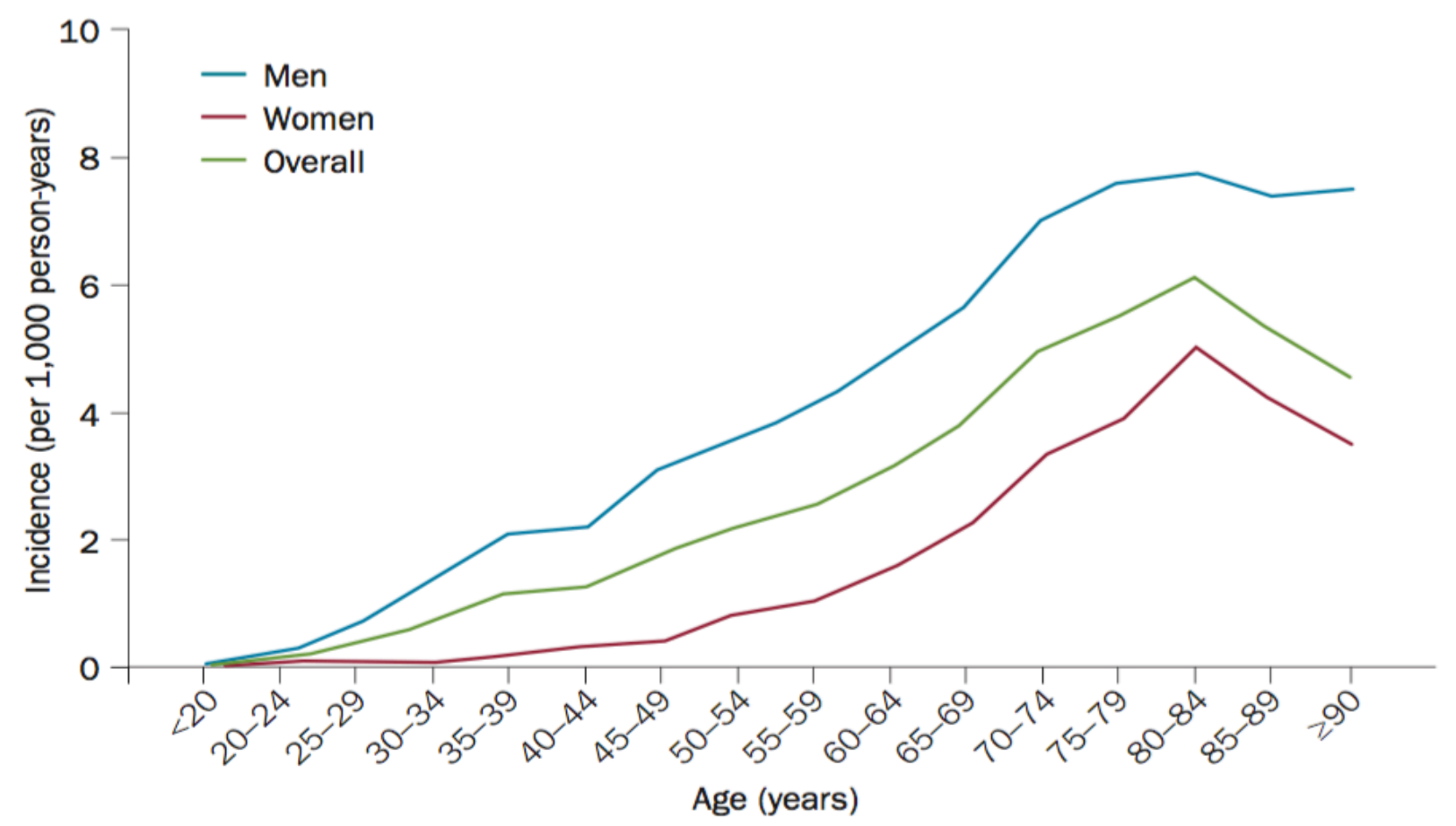

Figure 7. Age and gender specific gout incidence trend of UK in 2012 [20].

The risk of gout generally increases with age, as shown in Figure 8. This occurs because with age, renal function tends to reduce, resulting in under excretion of Urate, thereby increasing SUA levels as age progresses [18]. Additionally, changes occur in connective tissue with age, possibly making them more susceptible to crystal formation. 


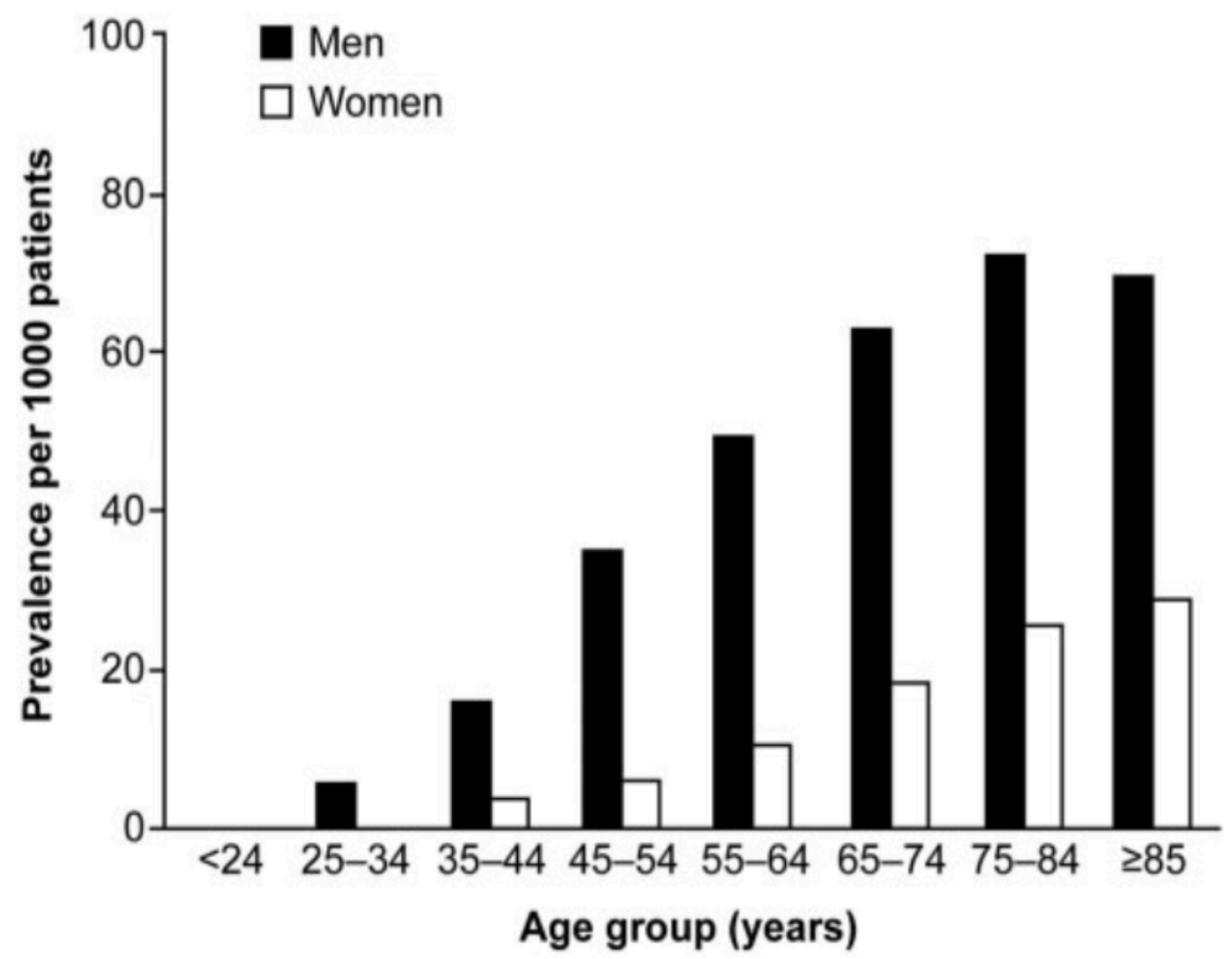

Figure 8. Age specific prevalence of gout in the UK for Men and Women [18].

\subsubsection{Diet}

Diet is a factor in the development of gout; numerous studies have indicated a relationship between purine-rich food and drink and gout [20]. Alcohol, high fructose drinks and red meat contribute to developing gout. In one study a relative risk of gout for sugar-sweetened cola was reported as 1.5 for men. A follow-up study reports a higher risk of developing gout for individuals with a high red meat diet [18]. Some alcoholic drinks, such as beer, are rich in purine, but less so than red meat. There are certain dietary factors that are negatively associated with the risk of gout, such as coffee, diary products, and dietary fibre. Vegetables, fruit and plant oils are also not high contributors to gout risk, as shown in Figure 9. 


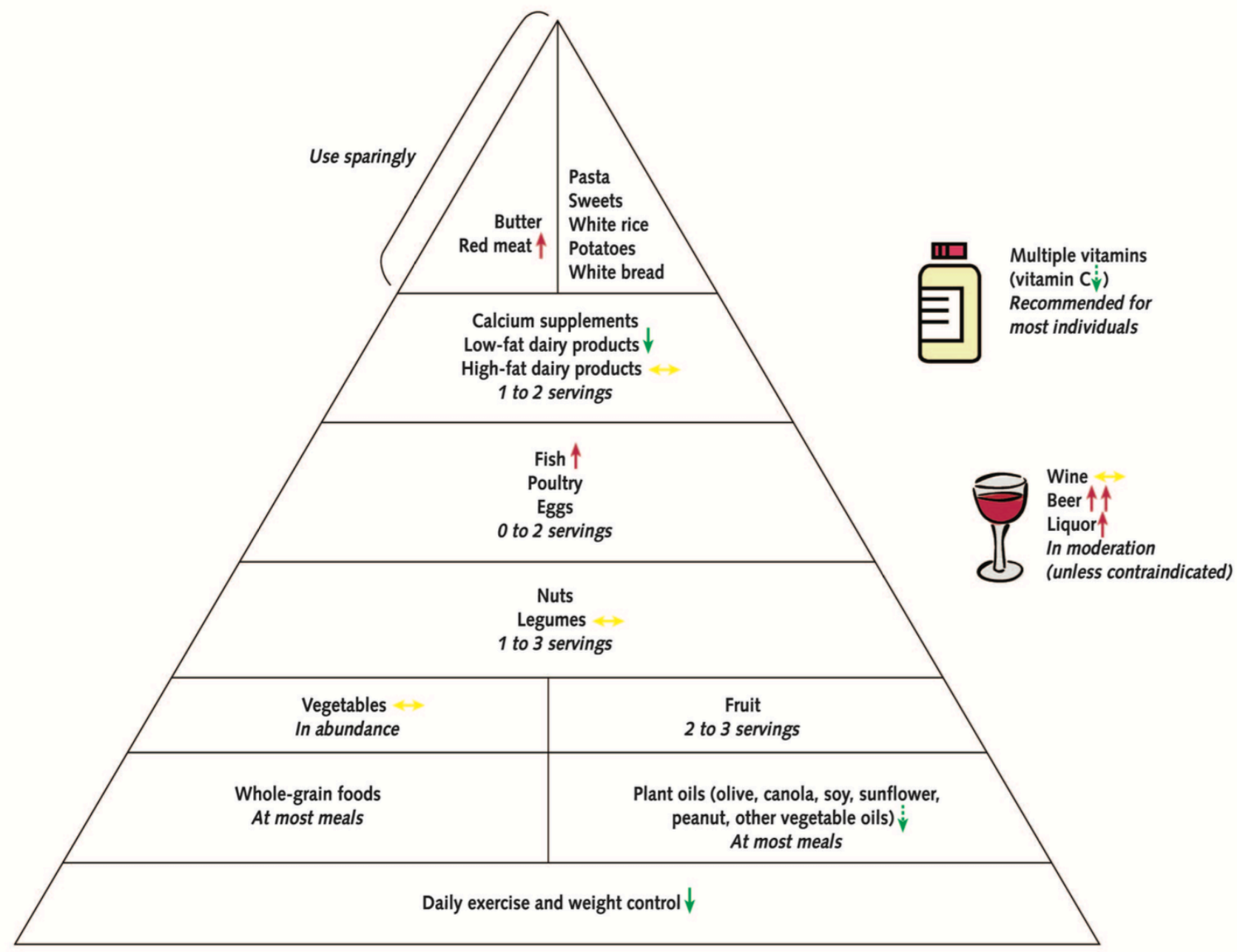

Figure 9. Harvard healthy eating pyramid for dietary influences on gout.

\subsubsection{Comorbodites of gout}

Conditions such as Hyperuricaemia, hypertension, obesity and insulin resistance are associated with gout, which are part of the metabolic syndrome [18], [20]. Obesity is associated with risk of gout, where cumulative risk of gout for women aged 70y was determined to be 11.8 $\%$ compared to $1.8 \%$, with $\mathrm{BMI} \geq 35 \mathrm{~kg} / \mathrm{m}^{2}$ and $\mathrm{BMI} \leq 25 \mathrm{~kg} / \mathrm{m}^{2}$, respectively. Risk of gout, for subjects with hypertension is two folds, while for subjects with chronic kidney disease have a 60 $\%$ increase in Risk of gout. Other comorbidities associated with gout are:

1. Renal disease [2]

2. Congestive heart failure

3. Anaemia

4. Psoriasis 


\subsection{Diagnosis of gout}

\subsubsection{Synovial fluid analysis (Gold standard)}

Synovial fluid (SF) analysis is the gold standard for detecting gout. It is used to confirm the diagnosis made from the clinical appearance of the affected joint. In SF analysis, needle aspiration is utilized to extract the synovial fluid from the affected joint, as shown in Figure 10. The extracted fluid is then analyzed with Polarized light microscopy (PLM), within six hours of extraction, otherwise the samples can be refrigerated [5], although refrigerating the samples can cause a reduction in the cell count, size and birefringence. Crystal concentration at room temperature decreases with time and when the fluid is refrigerated at $4^{\circ} \mathrm{C}$, but is more stable when refrigerated, which shows dissolution of crystals over time [25]. In a study analysing fluid samples from six gout patients, spontaneous crystallization occurred over time, resulting in the development of new crystals in unsealed slides [25]. Having a sealed tube or sealed slide prolonged the time it took for new crystal formation, but they formed nonetheless. This shows that if samples are first analyzed after substantial time from extraction, there is a higher likelihood of the test confirming gout.

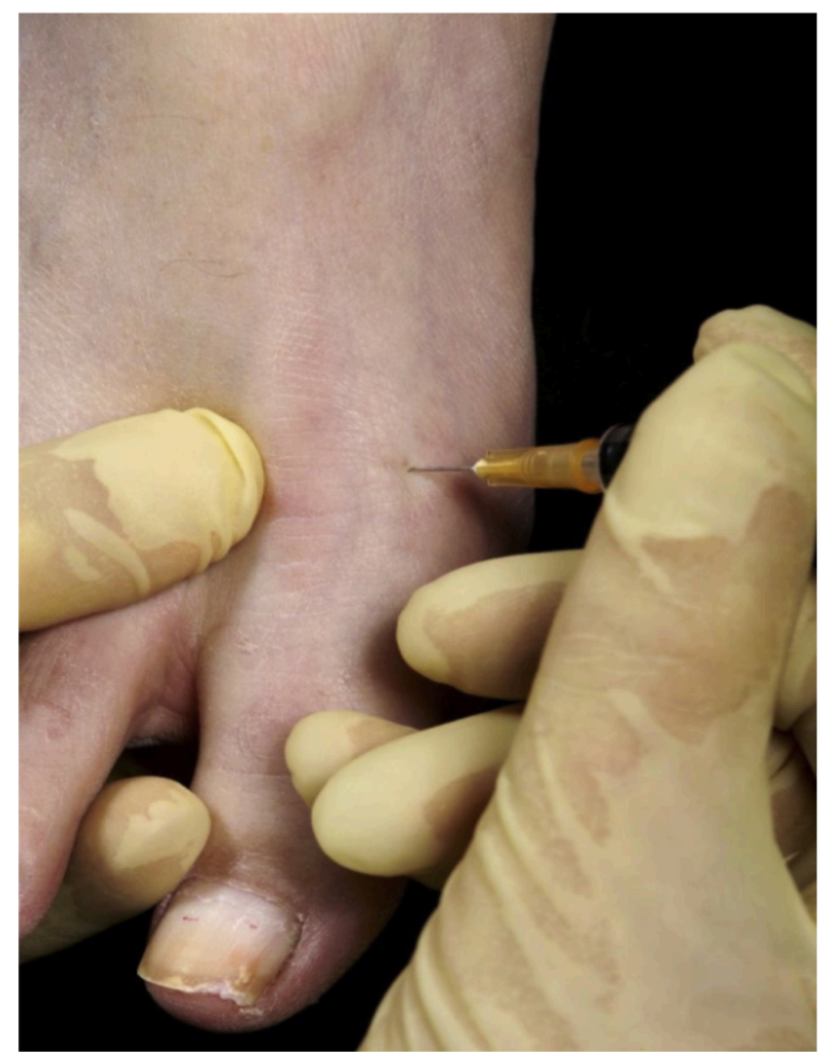

Figure 10. Needle asipiration procedure for the first MTP joint [26]. 
MSU crystals have a distinct needle-shaped structure, the length of which range from 2 to $20 \mathrm{~mm}$ [5]. A compensator is an optical device used to produce interference colors, these colors help identify crystals that are blue when perpendicular to the axis and yellow when parallel, as shown in Figure 11 [5]. In Figure 11, the crystals are intercellular; implying that the fluid belongs to a patient with acute gout, whereas, for a subject with chronic gout, the crystals would be extracellular.

Even though SF analysis is the gold standard, it has many drawbacks, such as the variability between examiners. The reliability of SF analysis has been evaluated in many studies [27]-[29]. In one study across 25 laboratories, 19 identified the crystals accurately [27]. Reproducibility has been recorded as poor, because of the importance of crystal concentration (weight of crystals per $\mathrm{ml}$ ) of synovial fluid in making a correct diagnosis [5]. It is difficult to identify crystals when their sizes are ultramicroscopic [30] or if the concentration is low, as they cannot be observed by microscopy, [31]. Therefore, lower crystal concentration is more likely to result in inaccurate results. Furthermore, compensated PLM can have average false-negative rates of approximately $30 \%$, which can be higher depending on how well trained the operator conducting the procedure is [4]. MSU crystals in SF can be missed in $25 \%$ of patients that have acute gouty arthritis [30].

Synovial fluid contains organic debris, which can also exhibit birefringence and block the view of MSU and Calcium pyrophosphate dihydrate (CPPD) crystals [31]. Furthermore, the aspiration makes SF analysis an invasive diagnosis technique, which causes pain and discomfort to the subjects. Aspiration requires many technical considerations before administration and it is difficult to find the correct position for administering the needle for aspiration [26]. Furthermore, there are some joints, such as the hip joint, which require imaging assistance to administer the needle. Generally, primary care providers do not attempt detecting crystals using this technique due to its high dependency on the observer, the need for trained personal and equipment that has lead to inconsistencies resulting in under or over diagnosis [4], [32]. Whereas, secondary care providers usually will as they have trained operators for crystal identification. Many clinicians use symptoms to make a diagnosis, but this results in a $30 \%$ false negative rate [4]. There is no simple automated method available that can determine the presence of the crystal. 


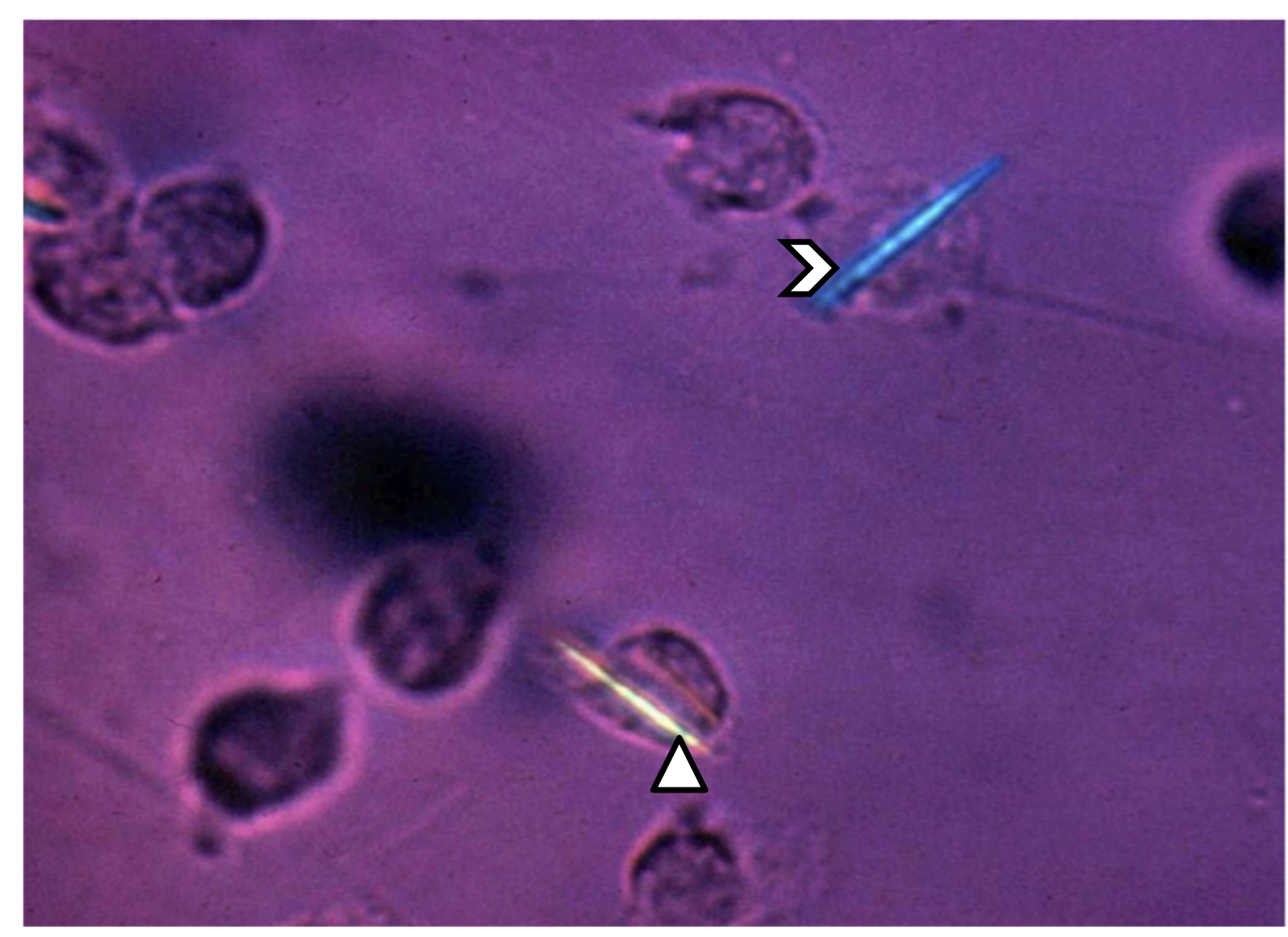

Figure 11. PLM image of MSU crystals, which are intracellular and needle-shaped objects coloured blue (arrow) and yellow (triangle) [26].

\subsubsection{Ultrasound}

Ultrasound (US) is a technique that utilizes sound waves and their subsequent reflections to visualize tissues [33]. This makes it equipped to detect crystalline materials within soft tissues and it has been utilized to detect uric acid renal stones and calcified gallstones [33]. Ultrasound is used to detect MSU crystals, as they reflect ultrasonic waves more strongly than surrounding tissue [33]. The features that help distinguish these crystals are the following in decreasing order of specificity towards gout:

1. Double contour signal (DCS), shown in Figure 12 [33], [34].

2. Tophus [34]

3. Hyperechoic depositions (HEDs), shown in Figure 13

4. Effusion, as shown in Figure 14

DCS is the most specific feature for gout, meaning it is only observed for subjects with gout. This represents MSU crystal deposition on the articular cartilage. Advantages of 
Ultrasound include repeatability, lack of radiation, low cost, high resolution and patient friendliness, due to being non-invasive. Furthermore, it is found to be more sensitive than conventional radiology for cartilage calcifications [33]. However, DCS has high specificity $(>=0.95$ ) but low sensitivity; ranging from $0.22-0.92$ over various studies [34]. The technique is operator dependent and requires a certified musculoskeletal sonographer [33]. Most studies conducted using this technique have been performed by experts in musculoskeletal US; it still remains to be seen whether the technique will produce the same level of accuracy when being assessed in real time without such expertise present. Another concern stems from evidence that US appearances of MSU crystals might appear in subjects with hyperuricaemia, without any other clinical manifestations of gout [34]. Suggesting that some US features might be overly sensitive to clinical disease, which might result in false diagnoses. Another issue is the time commitment when more than one area needs assessment [34].

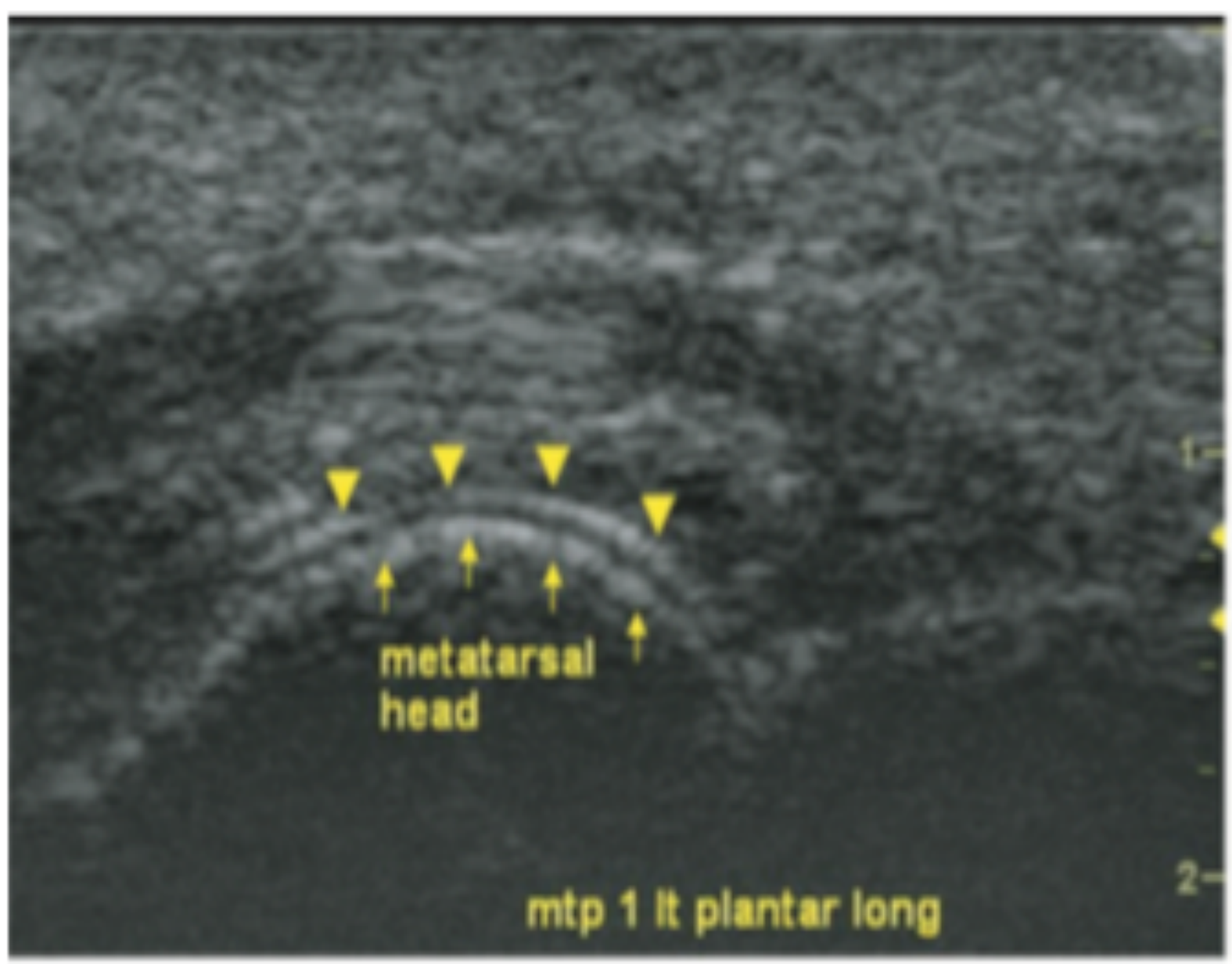

Figure 12. Ultrasound image of DCS feature labelled as metatarsal head [33]. 


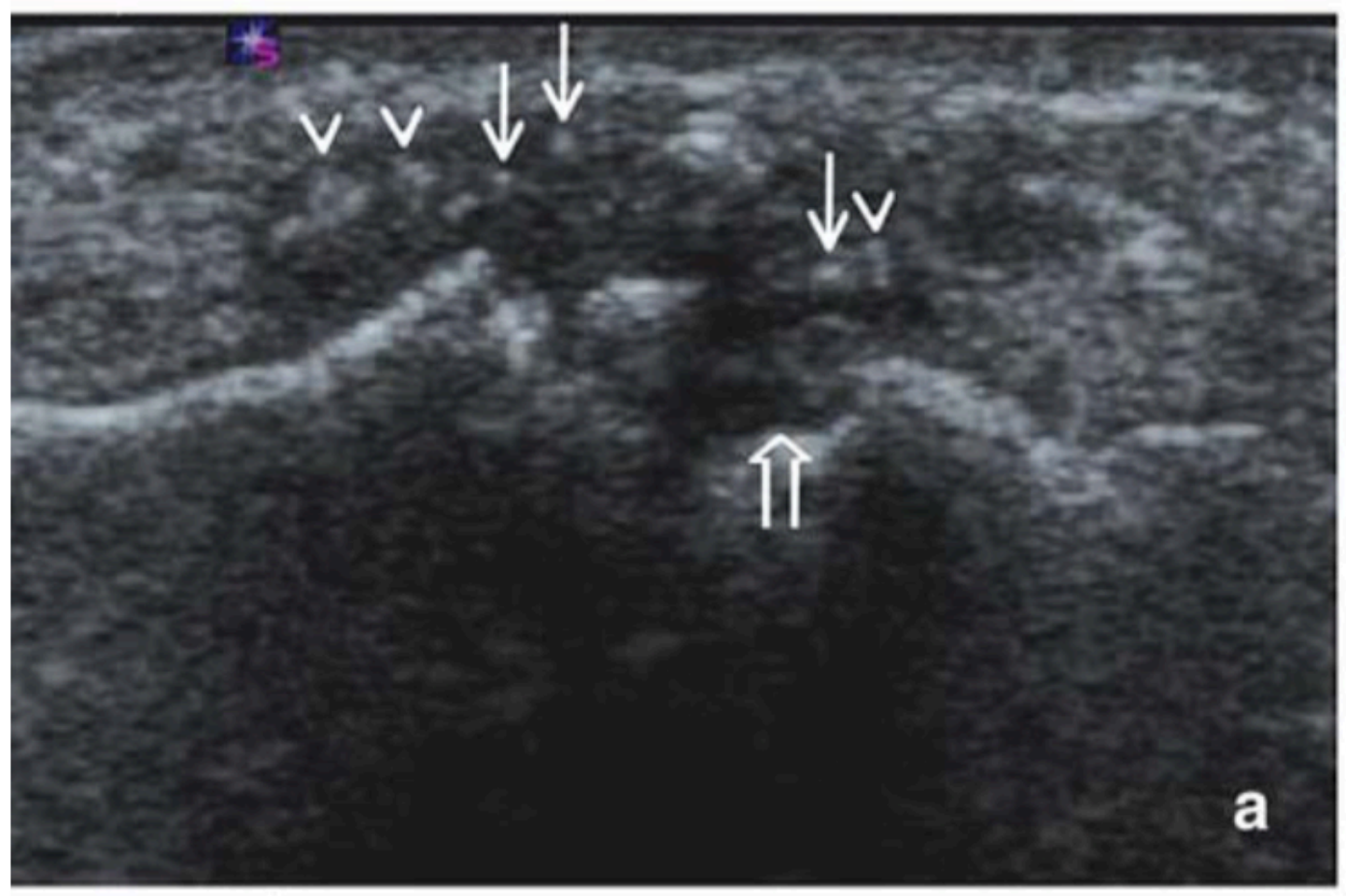

Figure 13. Ultrasound image of HED feature [35].

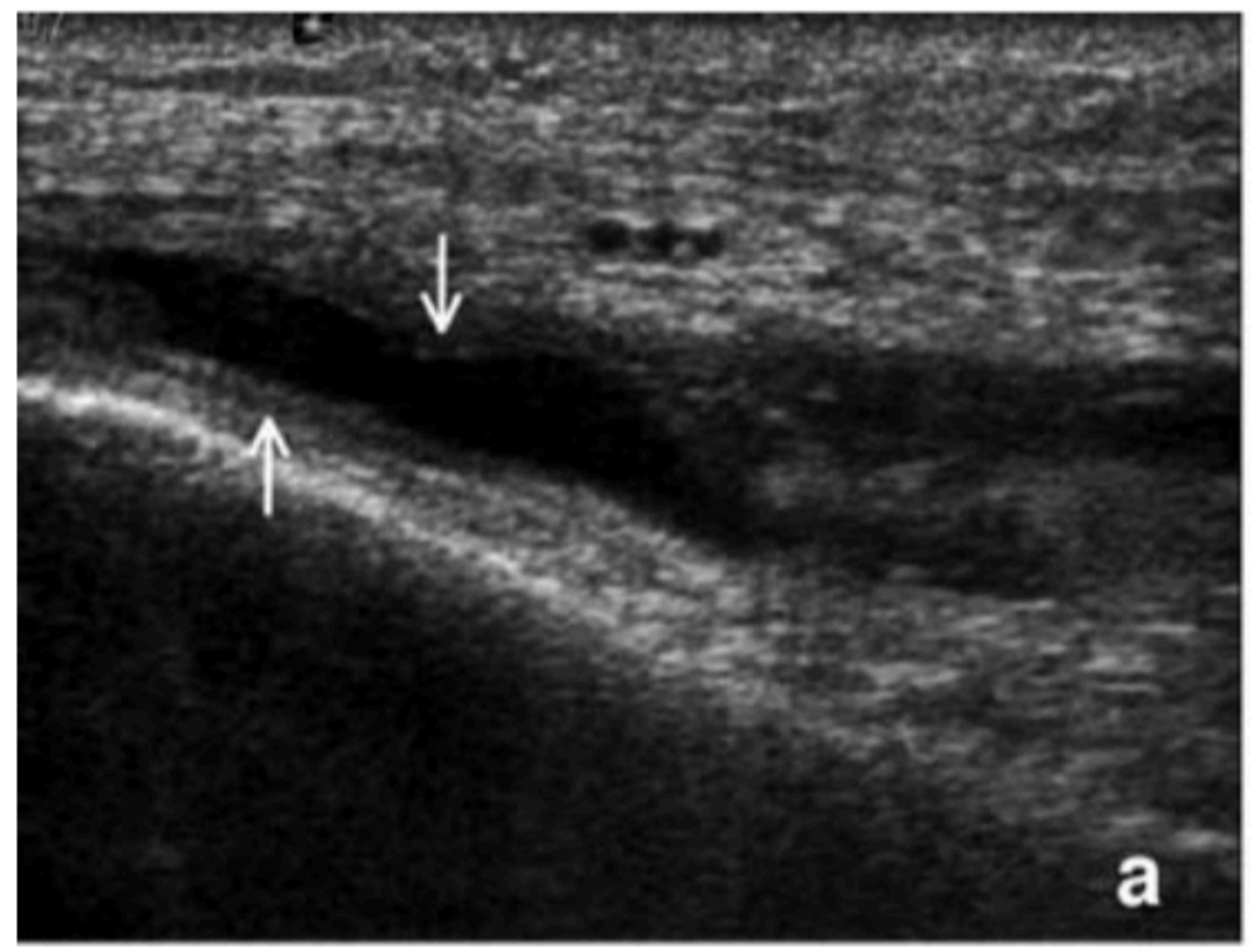

Figure 14. Ultrasound image of effusion feature (thin arrows) [35]. 


\subsubsection{Dual-energy computed tomography}

Dual-energy computed tomography (DECT) is a more advanced version of computed tomography (CT), in which two X-ray tubes are positioned 90 degrees to each other and their corresponding detectors, resulting in two datasets being collected [36]. In DECT, tissue is exposed to two different $\mathrm{x}$-ray spectra that exploit the photon-energy dependent attenuation of materials [37]. Thereby, DECT can differentiate between materials and provide volume measurements of those materials. These volume measurements can be utilized to monitor the amount of MSU crystals deposited at the affected site, and help clinicians identify the effect of urate-lowering drugs on patients, with respect to time. The appearance of MSU crystal deposits on DECT scans are shown in Figure 15. The sensitivity for DECT reported in some studies varied from 0.78 to 1 , while specificity varied from 0.79 to 0.95 . The false negatives from DECT were explained to be due to DECTs inability to register smaller MSU deposits, probably due to detection parameters of the DECT scanner. One study showed that DECT has moderate sensitivity for non-tophaceous gout and high sensitivity for tophaceous gout. Another study showed false negatives, which were attributed to subjects that had a disease duration of less than six weeks, meaning DECT has lower sensitivity for gout in its early stages [37]. Although, the sensitivity was lower for individual joints, where gout was proven with joint aspiration [38]. This means that joint aspiration analysis is able to detect gout at more joints than DECT. The major drawback of DECT is its high-cost, scarce availability and low sensitivity in the early stages of gout.
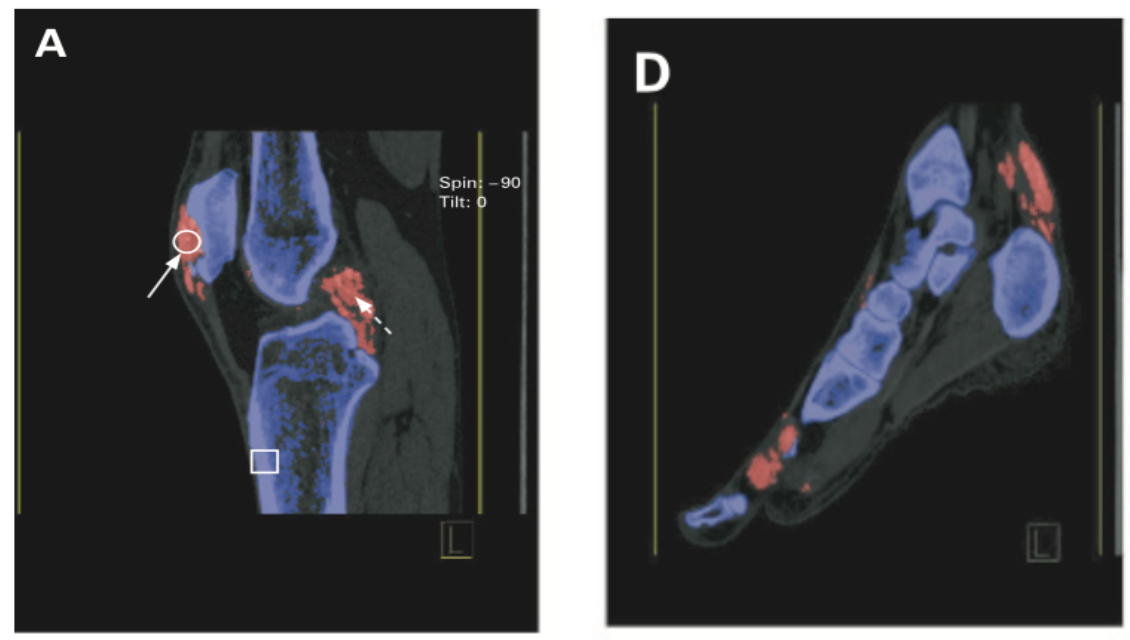

Figure 15. DECT images of MSU crystal deposits, which are coloured red. A knee and D foot [39]. 


\subsubsection{X-ray Conventional radiology (CR)}

$\mathrm{X}$-rays have been used to document a radiological manifestation of gout. The radiological manifestations that indicate gout occur only in later stages of the disease, such as chronic gout that are often known to be non-specific [40]. Only 1/3 of the cases provide sufficient information to the radiologist to make a diagnosis. Usually these manifestations are round or oval (commonly referred to as 'punched out') that indicates erosions around the target joint. These tend to be $1 \mathrm{~mm}$ to $30 \mathrm{~mm}$ in size with areas that are larger than $5 \mathrm{~mm}$ being indicative, but not wholly confirmatory, of gout [40].These radiological manifestations tend to occur 6-12 years after the initial acute attack [33]. This was the dominant method for gout diagnosis for almost a century [34]. The recent transition from traditional film radiography to digital detection systems has improved the dynamic range of the radiographs, which increases its contrast for soft tissues [34]. Inflammatory changes in synovium and bone are not well observed, along with MSU crystals [34]. The radiographs tends to be observed as normal for patients in the early stages of the disease [34]. X-rays don't allow the direct observation of MSU crystals, but instead show manifestations such as erosions around the joint. The technique has low sensitivity (31\%) and high specificity (93\%) when observing the following X-ray features: "defined as soft-tissue opacifications with densities between soft-tissue and bone, articular and periarticular bone erosions and osteophytes at the margins of opacifications or erosions" [34], [35].

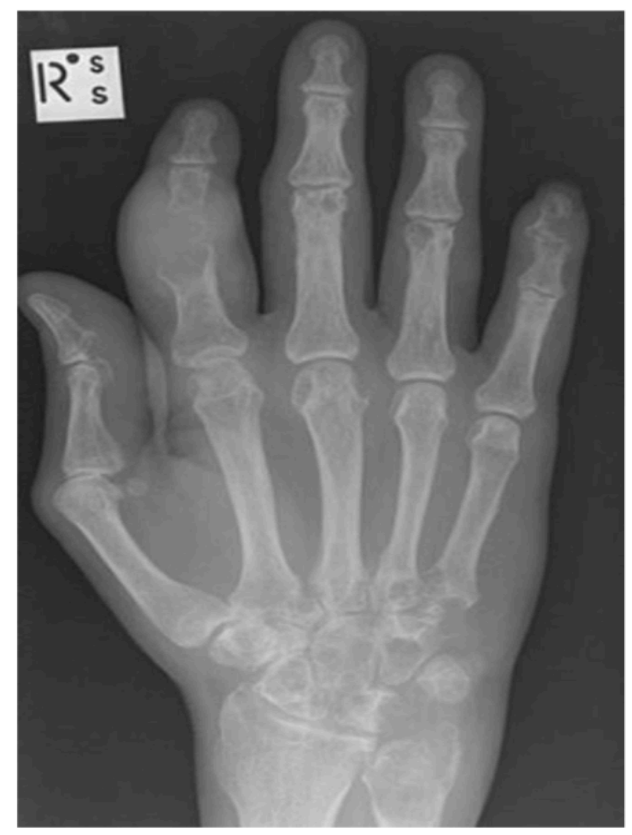

Figure 16. X-Ray image of gouty fingers with tophi on the index finger [34]. 


\subsubsection{Magnetic resonance imaging (MRI)}

MRI has been used to understand the formation of MSU crystals and progression of the disease, providing diagnostic clues [33]. MRI has facilitated early detection of tophi and erosions [41], where tophi tend to have low signal intensity $\mathrm{T} 1$ and $\mathrm{T} 2$ weighted images, which are images with different contrast parameters and with variable enhancement pattern. However, MRI has low specificity for gout. Other issues with MRI are its high cost, explaining its underutilization in the clinical assessment of gout [33], [37], [41].. MRI cannot specifically identify MSU crystals but rather detects abnormalities in soft tissue and osseous structures [37]. Ultrasound and MRI offer the ability to detect tophi and large crystal deposits but do not achieve an acceptable level of sensitivity and specificity [32]. MRI is better than US at identifying deeper structures; an image of a MRI scan for a knee depicting a tophi feature is shown in Figure 17.

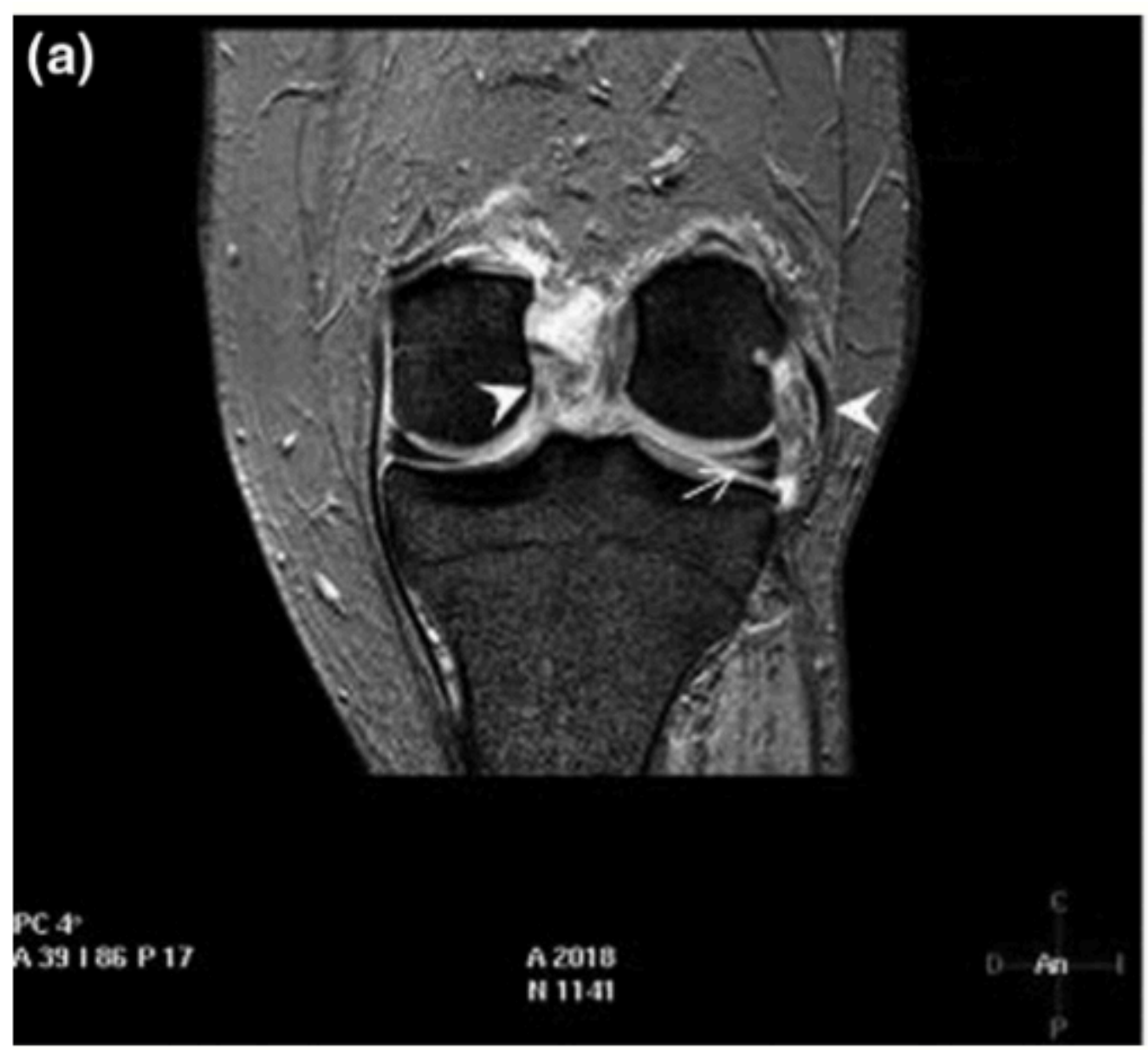

Figure 17. T2 weighted MRI knee scan with a tophi feature (wide arrow) [41]. 


\subsection{The need for a non-invasive diagnostic tool for gout detection}

The issues covered in this chapter show the need for a diagnostic tool, which can perform non-invasive measurements to determine the presence of MSU crystals automatically. Raman spectroscopy (RS) is a type of vibrational spectroscopy that is used for chemical analysis, which can give a biochemical fingerprint of a specimen [42]. RS has been used in vivo with fibre optic probes. It is also been used to identify synthetic MSU crystals [43] and MSU crystals in synovial fluid [4]. RS is a technique, which could facilitate diagnosis of gout without any invasion, it can also be automated and therefore would not be operator dependent. 
Chapter 3: Raman spectroscopy 


\subsection{The theory of Raman spectroscopy:}

\subsubsection{Basic theory}

Raman spectroscopy (RS) operates by the interaction of light with matter which results in absorption or scattering of photons [44]. Light is an electromagnetic wave (EW) consisting of two vectors: an electric vector and a magnetic vector [45]. These vectors are perpendicular to one another and to direction of propagation of the EW [45]. The electric field of the EW interacting with matter results in the scattering or absorption of light [45]. In addition, it is also possible that light might not interact with the target and pass through it [44].

There are several kinds of electromagnetic waves, with the major difference being due to the wavelength $(\lambda)$, the difference can also be attributed to the frequency $(v)$ [45], [46]. Wavelength, frequency and wavenumber $(\varpi)$ are interchangeable due to their proportional nature, as shown in (1) [44]-[46].

$$
\boldsymbol{v}=\frac{c}{\lambda} \quad \text { (1) }[44]-[46]
$$

Where $\mathrm{c}$ is the velocity of the light. In addition, the interaction between the molecule and electromagnetic field are discussed in terms of Energy. The relationship between the frequency and energy is as shown in (2).

$$
\boldsymbol{v}=\boldsymbol{\Delta} \boldsymbol{E} \boldsymbol{h} \quad \text { (2) [44]-[46] }
$$

Where $\Delta \mathrm{E}$ is the difference in energy between two quantized states and $h$ is the plank's constant. The inverse of Wavelength is Wavenumber $(\varpi)$, which is frequently used in spectroscopy that has a unit of $\mathrm{cm}^{-1}$, as shown in (3).

$$
\varpi=\frac{v}{c}=\frac{1}{\lambda} \quad \text { (3) [44]-[46] }
$$

The wavenumber and change in energy are directly proportional, as shown in (2) and (3). Furthermore, frequency is directly proportional to energy as shown in (2). These relations suggest that the highest energy level is associated with the highest frequency or shortest wavelength as shown in Figure 18 [44]-[46]. Further implications of these relations on the molecular vibrations and spectroscopic results will be covered in the following chapters. 


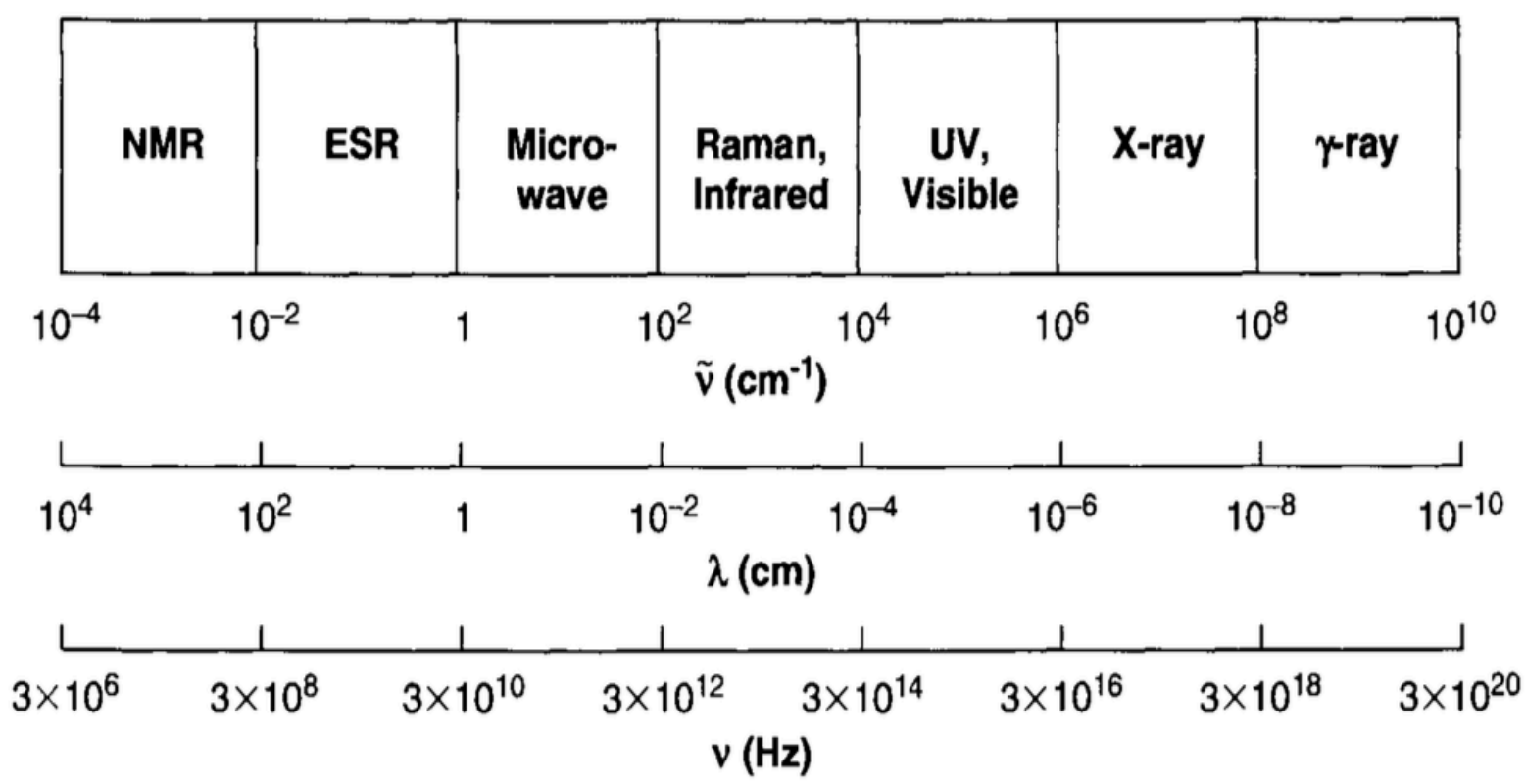

Figure 18. Electromagnetic spectrum and their associated parameters [46].

\subsubsection{Scattering}

When the electric field of the EW interacts with matter or compound, the electrons lead to the scattering of the incident light [45]. Scattered light originates from oscillating electrons, which are also a source for emitting radiation. These oscillating electrons are produced by the interaction between the electric field of the EW, which induces periodic vibrations in the electrons of the compound. The three basic types of scattering are: Rayleigh scattering, Stokes scattering and anti-stokes scattering. Rayleigh scattering is a form of elastic scattering [44]-[47]. Elastic scattering occurs when only electron cloud distortion is involved in scattering, in this instance the photons are scattered with very small changes in frequency. Rayleigh scattering is considered to be the dominant process in vibrational spectroscopy, where it has the same frequency or wavelength, as the incident light, therefore there is no change in frequency. Stokes and anti-stokes scattering are associated with the Raman effect and are explained in the following section.

\subsection{Raman scattering}

C.V.Raman and K.S. Krishnan first discovered light scattering a result of the Raman effect, in 1928, initially proposed as a "A new type of secondary radiation" [48]. In which, the authors proved the presence of modified scattering in addition to the unmodified scattering (X- 
ray scattering), the unmodified scattering being the result of the average state of atoms of molecules, while the modified scattering being the results of fluctuations from that average state. This modified scattering is what is referred to as Raman scatter or Raman effect that occurs in all directions [44]-[47]. For the Raman effect to be produced, the incident light needs to induce a change in dipole-moment or in molecular polarizability, that results in a change of frequency from that of the incident radiation [44]-[47], [49]. The resulting new frequencies are viewed as Raman lines or bands that collectively make up the Raman spectrum [47].

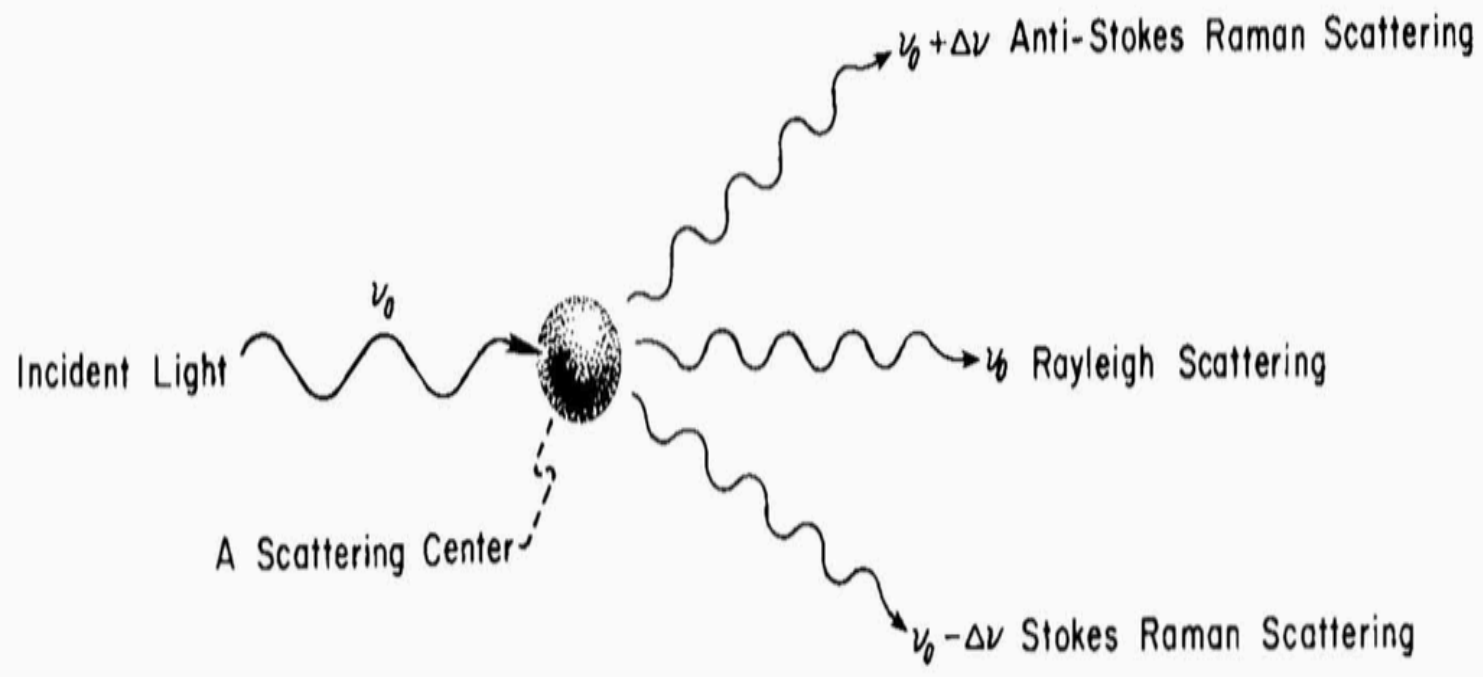

Figure 19. Illustration of the three different types of scattering [45].

Raman scattering is a result of the interaction between a molecules' electrons and incident light [44]-[47]. Where the transfer of energy occurs between the incident photon and scattered photon or vice versa, as a result the molecule's vibrational state changes, which also results in the scattered photon being different from the incident photon by one vibrational unit [44]. The process itself is very weak, as only a small amount of photons are Raman scattered, and therefore the exchange of energy has a much lower probability[44]-[47], [49]. As mentioned earlier, there are three basic types of scattering, an illustration of these types in shown in Figure 19. In Raman scattering the interaction between the light and molecule polarizes the electron cloud, inducing molecular vibration [44], [45]. This results in a temporary vibrational state called the virtual state as shown in Figure 20 [44]-[47], [50]. 


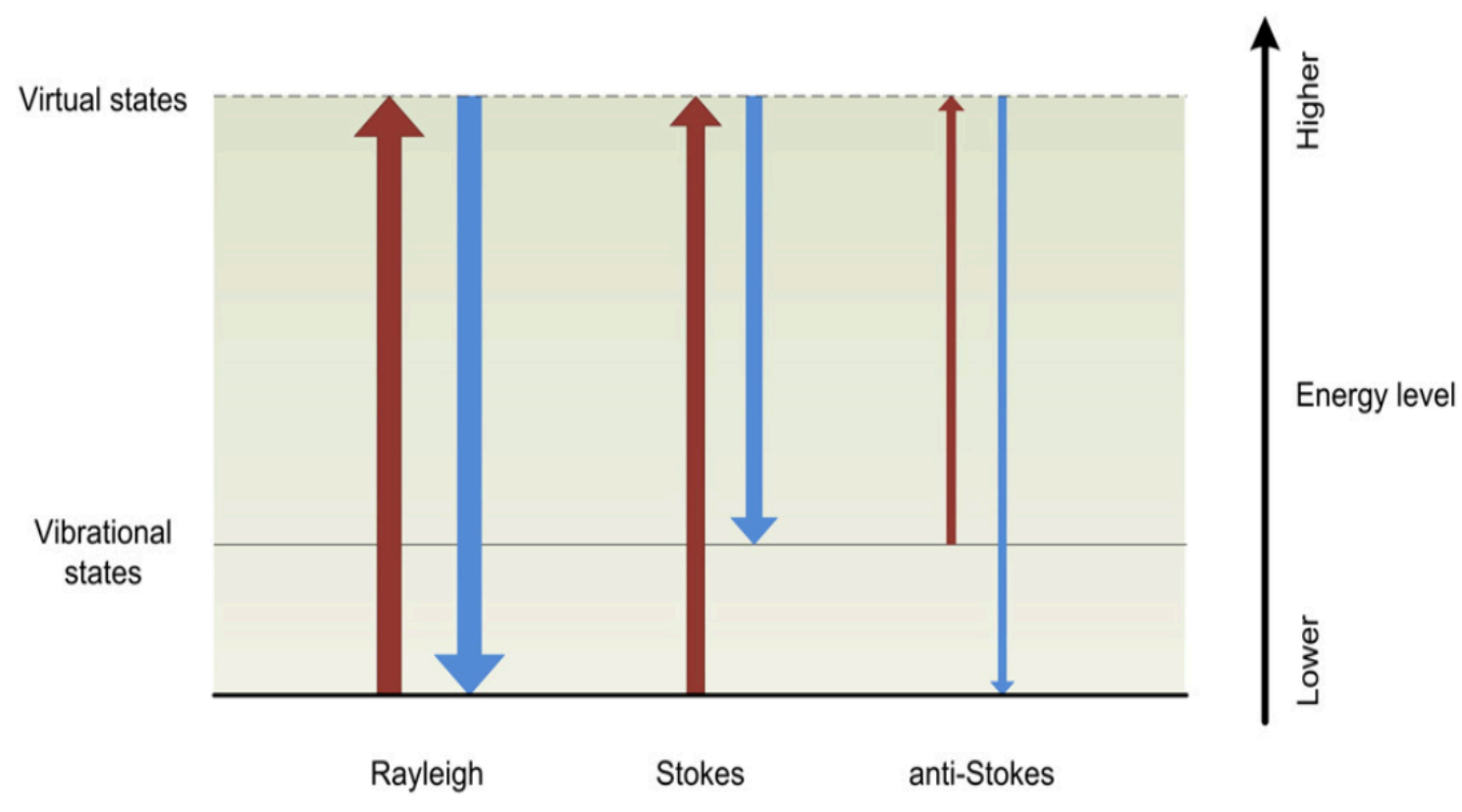

Figure 20. Energy levels for the three different types of scattering [50].

Raman scattering consists of both stokes and anti-stokes scattering that are forms of inelastic scattering [44]-[47]. If the change in frequency resulting from the molecular vibrations is $\Delta v$ and $v_{0}$ is the frequency of the incident light, the difference between them is Stokes scattering, whereas their sum is the anti-Stokes scattering. Stokes scattering is known to have lower frequency or energy than the incident light, where as Anti-Stokes scattering is known to have higher frequency or energy than the incident light [45].

In terms of molecular vibrations, the energy lost by the scattered light is equal to the energy given to induce the molecular vibrations, this is the stokes Raman effect [44], [45]. In Anti-Stokes, the energy is transferred to the scattered light from the molecule, this results in a gain in energy, and therefore the scattered light will have more energy than the incident light. In comparison, it is weaker then Stokes scattering and becomes weaker as the frequency of molecular vibration increases. The vibrational energy difference between the stokes and antistokes scattering are identical as the difference between $\Delta v$ and $v_{0}$ is identical as well [45]. In other words, they have symmetrical results, where the point of symmetry is the Rayleigh scattering, as shown in Figure 21 [45]. 
Raman scattering is expressed as a shift in energy or frequency from incident radiation to scattered radiation, which is usually referred to as $\Delta \mathrm{cm}$ or $\mathrm{cm}^{-1}$ [44]-[47]. Usually Raman scattering is observed on the Stokes scattered side, due to their higher intensity at low temperatures, but in certain instances anti-stokes is preferred [44]-[47], [49].

\section{Rayleigh}

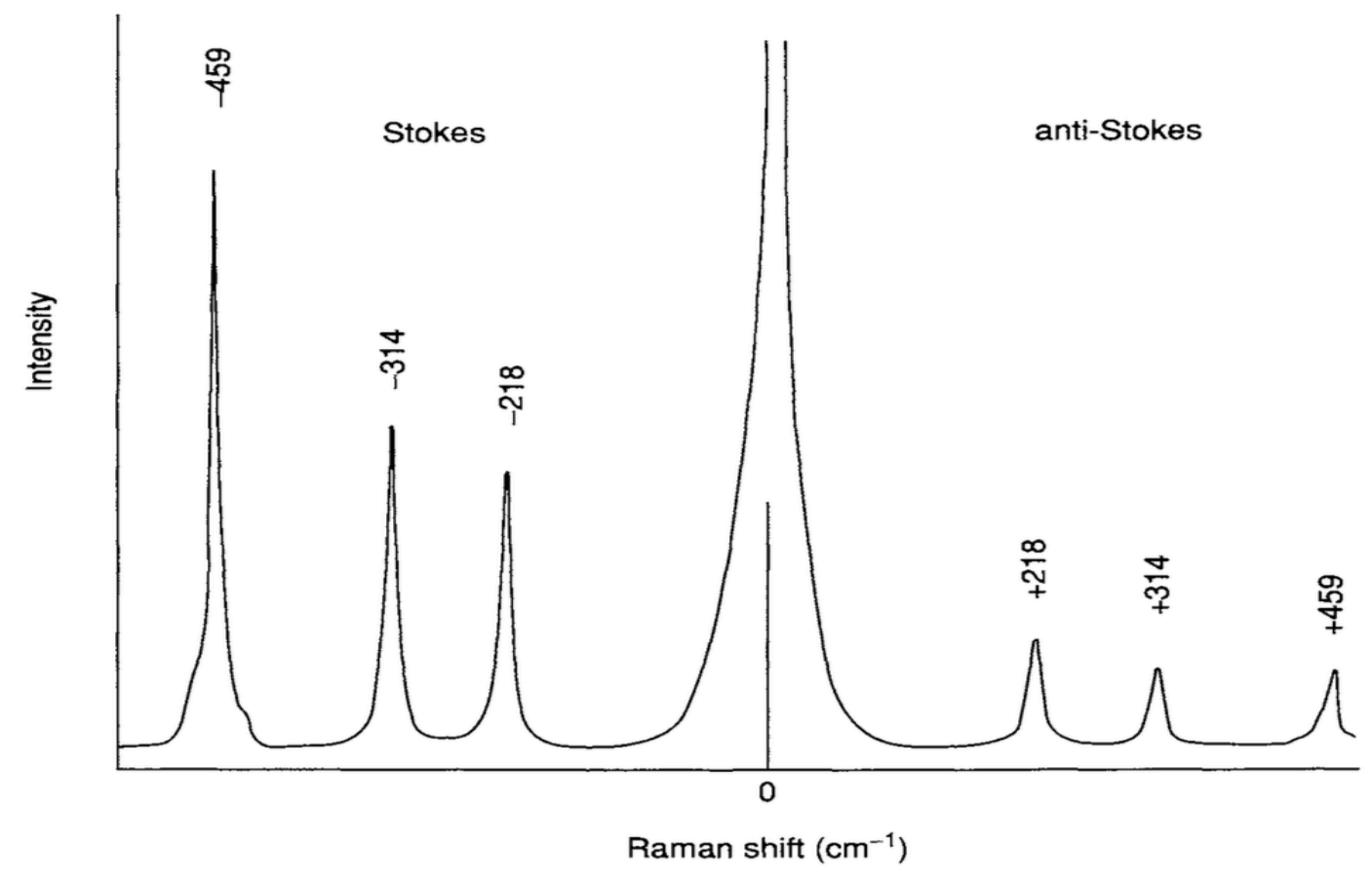

Figure 21. Raman spectrum of CCL4 with both Stokes and anti-Stokes scattering [46].

\subsection{Molecular Vibrations}

The Raman effect is produced when the incident light interacts with the molecule and induces a change in dipole-moment or in molecular polarizability, as it is vibrating, therefore a change needs to occur in the molecular vibrations of the molecule, as well as the shape of the electron cloud [44], [45], [51]. The distortion of the electron cloud, created by this interaction, is polarization [45]. A visual representation of polarization is shown in Figure 22. To properly interpret the Raman spectrum, one must understand the origin of the bands observed in the spectrum, therefore some understanding is required of the number of possible molecular vibrations. 


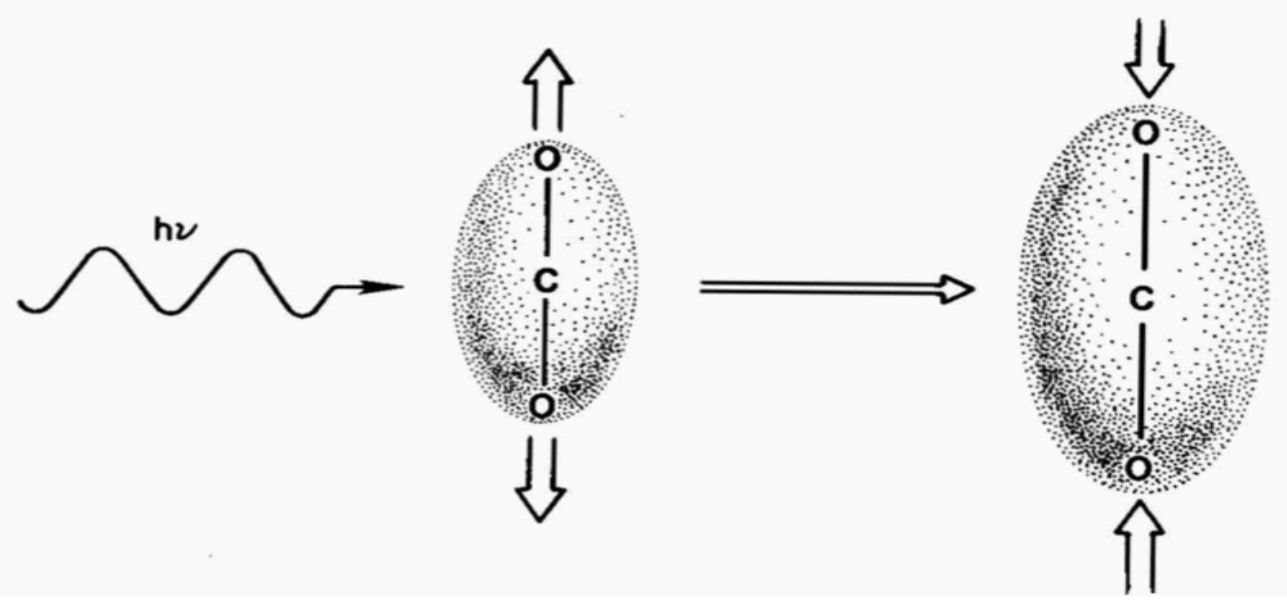

Figure 22. Visual quantification of polarizability of a $\mathrm{CO} 2$ molecule [45].

Vibration is an oscillatory motion, which is associated with a periodic change in position of atoms [45]. Vibration can be visualized by using a ball and spring drawing, where the ball represents the atomic nuclei and the spring between them represents their interatomic bond [44], [45]. The stretching of the spring and its release, results in a stretching vibration, which is the vibration occurring in Figure 22. Molecular vibrations are not limited to just bond stretching, but bending vibrations are also possible. The strength of the bond and frequency are related as such that higher the bond strength, higher the frequency. A lowering of frequency is related to the mass of the atoms, as heavier atoms will have lower frequency [44].

In assigning vibrations to peaks in a Raman spectrum, one must be aware of the interactions between bonds which are close together and of similar energies, as they can interact to create a vibration of a group of atoms [44], [45]. This vibration is what is observed in a Raman spectrum. It is not always the case that bonds of a molecule are closer together and have similar energies, as in some cases they can be far apart and have a large difference in vibrational energy [44], [45], [51]. In such a case, larger molecules can be simplified by separating them into groups and normal modes of vibrations [44]. Raman bands for specific bonds or groups are known as group frequencies [51].

The total degrees of freedom of a molecule with $\mathrm{N}$ number of atoms are $3 \mathrm{~N}$ degrees of freedom (DOF), where $3 \mathrm{DOF}$ are reserved to describe the translation of a molecule, and a 
further 3 reserved for the rotational movement of a molecule (or only 2 DOF for linear molecule) [44]-[46], [51].

Some of the possible vibrations are: Symmetric stretching, symmetric breathing, asymmetrical stretching, bending or deformation, twisting, rocking, and wagging [45], [51]. For bending vibrations, they can be in plane or out-of-plane [45]. As mentioned earlier, in the case for Raman scattering to be active, polarization of the molecule must occur, for which, symmetric vibrations need to occur, as asymmetric vibrations results in very weak or non-existent Raman scattering, but this is not always the case because of the mutual exclusion rule [44], [45], [51]. The rule states that if a molecule has a center of symmetry, then no vibration can be both Raman and infrared active, thereby, only one can be active at a time or both will be inactive. An example of stretching vibration and bending vibration of $\mathrm{CH}_{3}$ of the methylene group are given in Figure 23 and Figure 24, respectively.

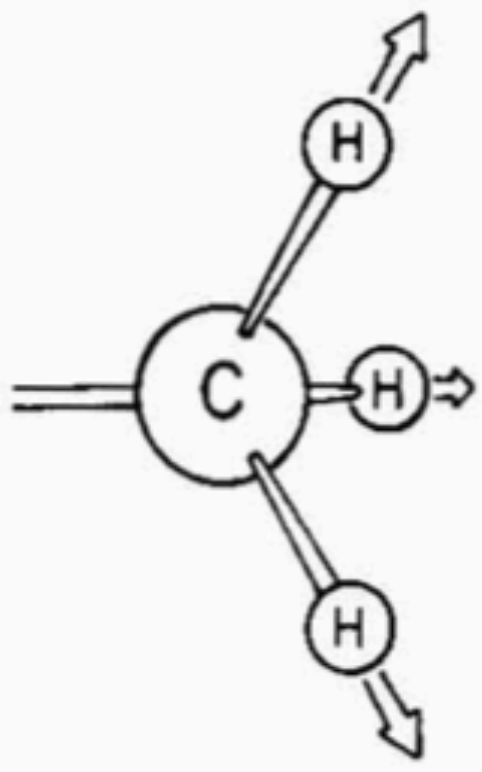

Symmetric

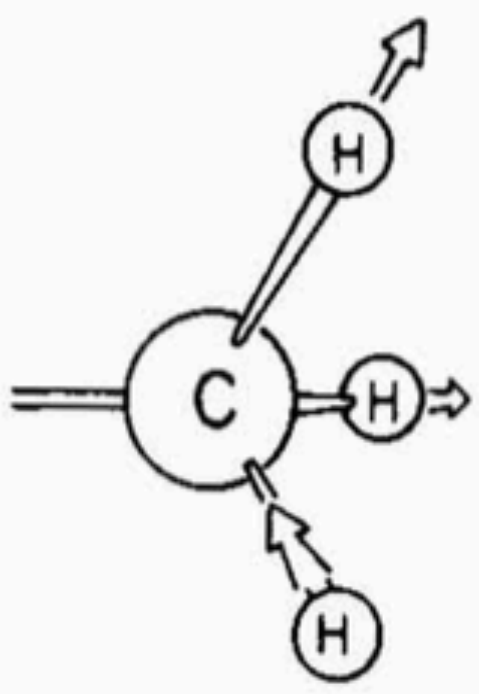

Asymmetric

Figure 23. CH3 methyl group stretching vibrations [45]. 


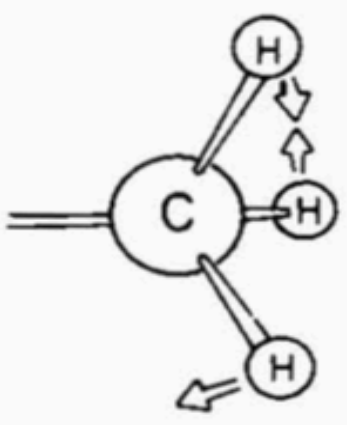

Asymmetric

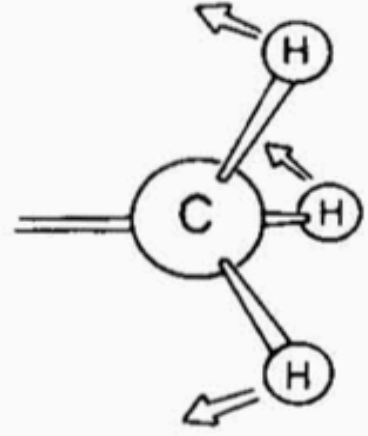

Symmetric (Umbrella)

Figure 24. CH3 methyl group bending vibrations [45].

The type of vibration is affected by the molecular structure, the strength of the bonds, the number of atoms, the atomic mass and whether the vibration occurs in plane or out of plane [44], [45]. All these factors affect the resulting vibration, which also affects the frequency. For example, as shown in Figure 25, for the same molecule, different frequencies can occur, as out of plane twisting occurs at a different band of frequency, as compared to symmetrical bending. Thereby, it is imperative to have a background into all factors that affect the occurrence of Raman scattering and its corresponding frequencies.

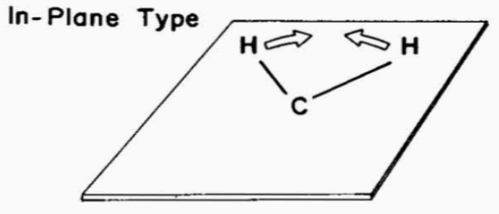

Symmetrical Bending

(scissors deformation)

$1463 \mathrm{~cm}^{-1}$

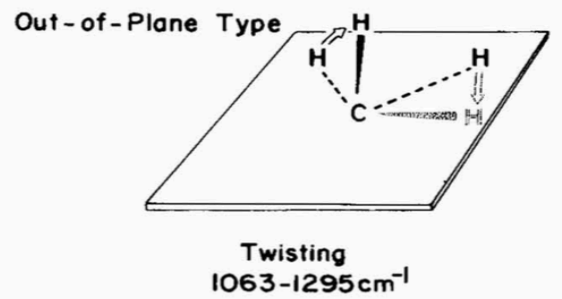

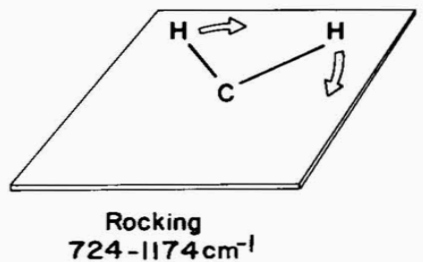

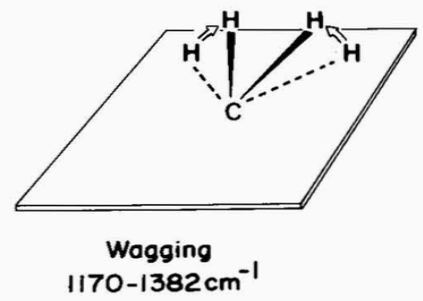

Figure 25. In plane and out plane bending vibrations of CH2 of the methylene group [45]. 


\subsection{Raman experiment}

A Raman spectrometer must consist of [44], [46], [51]:

1. An excitation source

2. Sample preparation and illumination

3. Wavelength selectors (wavelength sorting device)

4. Detection system

5. Spectral data processing

\subsubsection{Excitation sources}

The selection of the excitation source for a Raman spectrometer is very important, as Raman scattering is dependent on the fourth power of frequency, thereby using a higher frequency laser will increase the Raman sensitivity and improve the scatter [44]. Using the highest frequency would mean operating in the ultraviolet (UV) region. An advantage of this would be the reduction in fluorescence, a major issue in RS as it occurs at energies that are below the excitation energy, much stronger than Raman scatter, and is intense in the Stokes scattering region of the spectrum. This ends up making it difficult to read the spectrum and identify Raman lines. A disadvantage of using a UV excitation source is the high possibility of sample degradation from burning. For the reasons mentioned above it is important to understand the requirements of the application, to aid in choosing the right instrument.

Usually excitation sources used for RS are plane or linearly polarized, or at the least will be partially polarized, this is done by having a optical component called the polarizer [44]. There are many excitation sources available to choose from, the most common choice for Raman systems tends to be a laser in the visible region. The types of excitation sources available are the following [44]:

1) Visible excitation

i) Continuous wave $(\mathrm{CW})$ gas lasers

ii) Diode lasers

iii) Tuneable lasers

2) Near infrared region (NIR) excitation

i) Neodymium-YAG lasers (Nd:YAG) 


\subsubsection{Sample Preparation and illumination}

RS is known to require minimal sample preparation, but there are some samples that require some preparation, even though a large amount of homogenous materials can be examined with minimal preparation [44]. Many materials, organic and inorganic are known to be suitable for Raman spectroscopic analysis. The forms of materials can be solids, vapours, liquids or even powder form. Depending on the arrangement, the angle of the sample to the scattered beam can have orientation effects, such as for crystalline samples. Rotating the sample can average out these orientation effects.

The use of fibre optics for sampling is also employed in RS [44]. Fibre optics is a pipe used to transfer optical signals from and to a sample. For RS a fibre optical can have a single fibre to carry the excitation laser beam to the sample and have single or multi-fibre cable to carry the Raman scattered signal back to the spectrometer. Fibre optic Raman probes are equipped with a notch filter placed between the sample and the scattered single returning from the sample. This is done to reduce the dominant Rayleigh line, that is stronger $\left(\sim 10^{6}\right.$ stronger $)$ than the stokes region of the spectrum [46].

\subsubsection{Wavelength selectors}

RS requires the imaging of the focused laser beam onto the detector, for this reason a wavelength-sorting device is required, which essentially is used to select the wavelengths that are desired to reach the detector [51]. Two basic sorting devices that are available are: Monochromators in dispersive systems and interferometers, in both cases this is achieved by collecting the light and focusing onto the sorting device. RS requires a dispersive system that is capable of eliminating the strong Rayleigh line while analyzing the comparatively weak Raman scattered radiation [46], [51].

\section{5-Detection of light.}

Raman signals are inherently weak, thereby presenting significant problems with detection [46]. There are a number of techniques that are employed, Firstly, photon counting essentially converts photons into electrical signals, a drawback of the device being that its suitable for most Raman signals, except for the strongest ones. Secondly, photodiode array, a multichannel photon detector with an array of photosensitive devices that convert an image into a 
charge pattern, but these are not typically used owing to their lack of sensitivity to Raman signals. Lastly, the charged couple device (CCD), the most commonly used detection device for RS. A CCD consists of an array of photosensitive elements (pixels) on a semiconductor, where each pixel upon interaction with a photon generates photoelectrons to store as a small charge. The charge is a function of the number of photons striking a pixel. CCD is most commonly used due to its low readout noise and high sensitivity to a wide range of wavelengths.

\subsection{Spectral data processing}

The spectral data obtained in certain situations requires enhancements to improve the appearance of the spectrum, thereby to ease the process of interpretation, but great care needs to be taken while enhancing data, as it can lead to loss of data [44]. There are several data handling softwares available for manipulating spectrum that can aid spectroscopists in removing fluorescence. Some other tools that can be used are: baseline flattening, deconvolution, and smoothing. The smoothing function can be used for noise reduction if the data has a low signal to noise ratio, otherwise care needs to be taken as high order of smoothing can lead to complete removal of bands.

\subsection{Spatially-offset Raman spectroscopy (SORS)}

SORS is a technique, which can obtain spectra for sub-layers and sub-structures of specimens [52]. The laser illumination zone is spatially offset from the collection zone. This allows for the collection of spectral info from different depths. This technique is usually coupled with a fibre-optic probe for illumination and collection purposes. After collection, numerical processing is used to recover the spectral data at different depths within the tissue. The surface layer can be subtracted from the spectra by obtaining signal at two offsets, one a zero-offset and the other non-zero. Zero-offset is the spectral data for the surface layer. SORS is capable of obtaining data at depths greater than $500 \mu \mathrm{m}$, where as confocal Raman spectroscopy cannot [52]. This technique has been utilized for obtaining transcutaneous scans for mice bone with a custom built SORS device[53]. The technique has also been used for detecting breast cancer lesions noninvasively, determining cancer margins and glucose detection[52]. 


\section{Chapter 4: Raman spectroscopic diagnosis}




\subsection{Cancer diagnosis}

RS has been evaluated as a diagnostic tool for lung, skin, colon, cervical and breast cancer [54]-[62]

\subsubsection{Breast cancer}

RS has been used for sentinel lymph node (SLN) assessment, which plays a significant part in treatment protocols for breast cancer [42]. Traditionally, the axillary node samples obtained from this biopsy are sent to histopathology, which can take up to ten days for full analysis, and if the analysis reveals the SLN to be positive, then an axillary clearance surgery or radiotherapy needs to be performed. The waiting period for full analysis causes patient stress and the healthcare provider treatment delays and cost implications. However, RS provides an immediate analysis for intraoperative assessment of SLN in the operation theatre without the need to send the samples for histopathology. RS was assessed against histopathology; scans for positive and negative nodes were obtained with RS and spectral analysis was performed using principal component analysis (PCA). RS was able to differentiate between the two groups, with a specificity of $99 \%$ and a sensitivity of $97 \%$, which are comparable results to other intraoperative assessment techniques, such as endoscopy [42]. In addition, recent studies have shown RS can discriminate between normal, benign and malignant breast cancer tissue, due to their different chemical compositions [42], [61].

\subsubsection{Colorectal cancer}

A fibre optic probe RS has been utilized as a tool for diagnosing dysplastic lesions that have malignant potential. Currently, colonoscopy is used to identify such lesions, a technique which is not capable of viewing microscopic dysplasia, prompting the need for multiple biopsies. Furthermore, it is difficult to differentiate between malignant and benign lesions [42]. A novel design of a confocal Raman optic probe that fits within the endoscope channel has been developed [42], [63]. In-vivo and ex-vivo studies have been conducted; an in-vivo study was performed on 30 patients, measuring normal and dysplastic lesions and using a principal component fed linear discriminant analysis (LDA) that resulted in a sensitivity of $94 \%$ and specificity of $96 \%$ [42]. Ex-vivo studies have also showed a high ability to discriminate between different types of polyps. 


\subsubsection{Oesophageal cancer}

RS has been utilized as a fibre-optic diagnostic tool to help identify pre-cancerous dysplasia, which can develop in the oesophagus. The current technique of using white light endoscopy to identify such tissues is difficult for even the most experienced physicians, yet the need for early detection is crucial [42]. RS was utilized to detect this precancerous dysplasia invivo, where oesophageal cancer tissue samples were discriminated against normal tissue sample from the oesophagus with a sensitivity of $97 \%$ and specificity of $95 \%$ [42]. Ex-vivo studies were also conducted but more investigation is required to investigate the effect of acquisition time on the collection efficiency of RS scans.

\subsubsection{Lung cancer}

A study conducted by a group in Japan on fresh human lung tissue, evaluated the possibility of a NIR multichannel Raman spectroscope at $1064 \mathrm{~nm}$ of wavelength, discriminating between normal lung tissue and cancerous lung tissue [58]. The results showed an increase in the intensity for the $1659 \mathrm{~cm}^{-1}$ Raman peak for Amide I, which is sensitive to cancer development. Another study used RS coupled with multiple bronchoscopy techniques, to perform in vivo measurements, which reduced the number of false positives in conventional lung cancer detection techniques with a specificity of $91 \%$, where on average large clinical trials showed a specificity of $60 \%$ due to high number of false positives [64].

\subsection{Diabetes diagnosis}

Studies have been conducted to test the ability of using RS in monitoring glucose levels non-invasively in diabetic patients. A study conducted on animal models, with the goal to test the ability of RS as an in vivo quantification of blood glucose levels, by focusing a laser on the blood vessels through the skin, the resulting spectra from the control subjects were observed to establish a relationship between the glucose concentration and Raman intensity [65]. The glucose concentration was determined by calculating the ratio of peak height for glucose against hemoglobin, where the results showed a relationship between the Raman intensity and glucose concentration, with a linear correlation coefficient of 0.91 thus showing that the method can be used for non-invasive quantification of Blood glucose levels. Another group of researchers were reported to have scanned human aqueous humor with RS to determine glucose levels [66]. The analysis of human vitreous fluid with RS was also reported to have been used to distinguish 
between a diabetic specimen and a control [67]. Furthermore, in a preliminary clinical study a Raman spectrometer was used to observe the compositional changes in patients' bones with diabetic osteomyelitis, From the study they were able to identify markers, such as dicalcium phosphate dehydrate and uncarbonated apatite, for the early stage detection for the disease [68].

\subsection{Raman spectroscopy of human bone}

RS has been used to study the chemical composition of human bone [69]. Tools such as $\mathrm{X}$-ray and electron microscopy require sample preparation that can alter the bone compositions. In a study Fourier-transform Raman (FTIR) spectroscopy was used to successfully obtain a RS scan of human cortical bone, which had no florescence whatsoever and is shown in Figure 26. The most significant peaks were: $422 \mathrm{~cm}^{-1}, 583 \mathrm{~cm}^{-1}, 960 \mathrm{~cm}^{-1}, 1270 \mathrm{~cm}^{-1}$ and $1450 \mathrm{~cm}^{-1}$. A recent study was conducted on Murine bone in-vivo to obtain a transcutaneous RS scan, in which they reported the use of a fiber-optic Raman spectroscope developed specifically for use in mice [53]. The RS device designed for the study used the spatially offset technique, as this facilitates transcutaneous measurements, removing contributions from skin layers, this technique is discussed further in 3.7 Spatially-offset Raman spectroscopy (SORS). A second study used deep ultraviolet RS to successfully obtain scans of human cadaver bone [70].

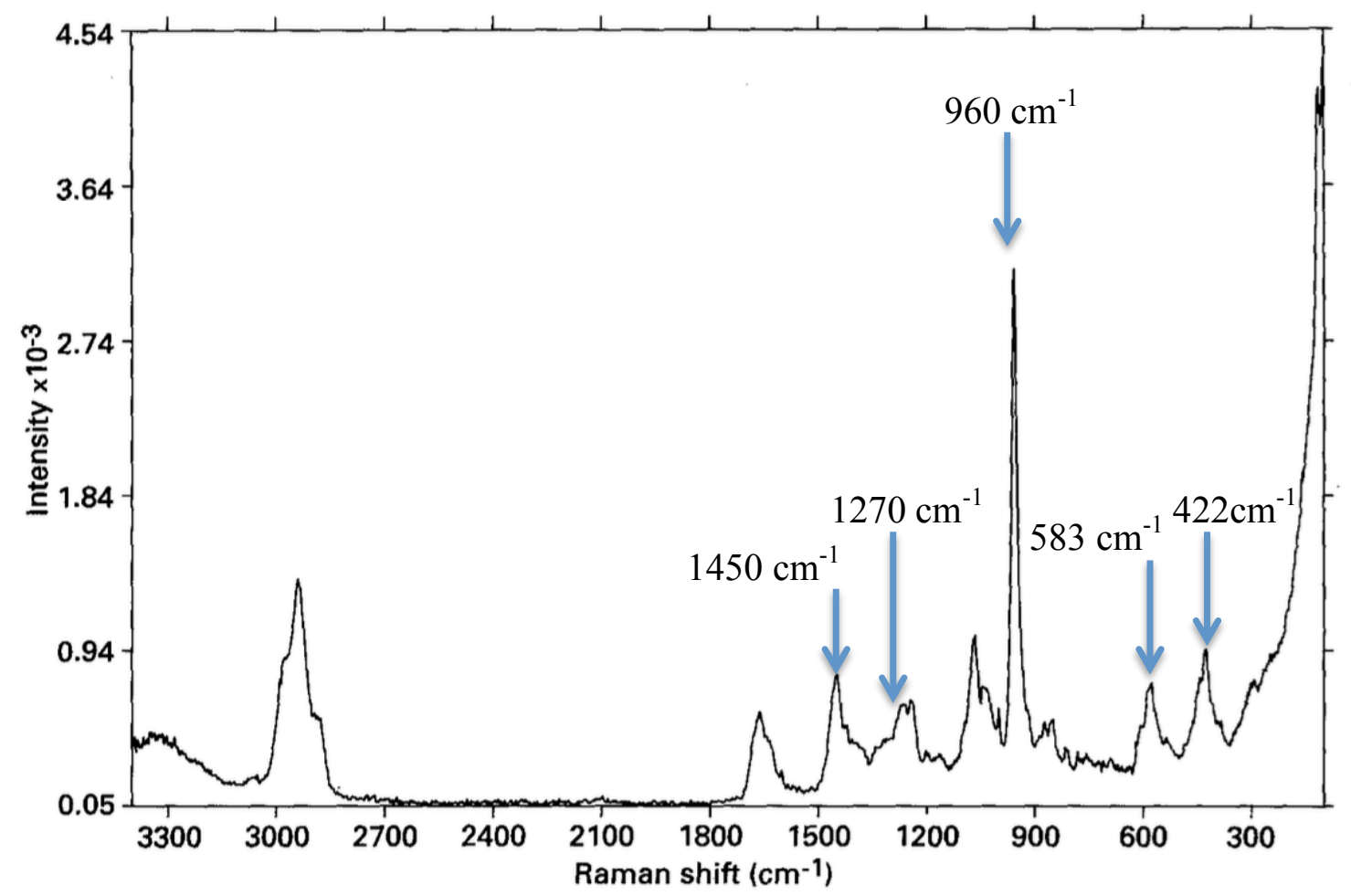

Figure 26. RS scan for whole human bone, obtained using a Fourier-transform Raman spectroscope [69]. 
RS has also been used to determine bone quality focusing on affects of certain factors on composition, factors such as age, osteoporosis or abnormal bone composition from exposure to radiological or chemical agents [71]-[75].

\subsection{Raman spectroscopy for diagnosing Osteoporosis and screening for fracture risk}

RS can assist in the discrimination of fracture risk in patients that have osteoporosis and those that do not [75]. Osteoporosis results in the loss of bone, weakening its structural integrity[75]. This is why osteoporotic bone is weak and more likely to fracture. The purpose of the study was to find a link between the mechanical and chemical properties of bone and nail, where RS assessed the chemical properties. RS scans were obtained from nail clipping of patients with and without osteoporosis, where the focus was on the disulphide bond (S-S) peak at $510 \mathrm{~cm}^{-1}$, as shown in Figure 27. Non-osteoporotic patients had a sharper S-S peak than osteoporotic but a smaller half-width maximum. Another study that expanded on this technique, showed that the S-S content was lower in osteoporotic patients [74]. In this study, carbon sulphide bond peaks of $621 \mathrm{~cm}^{-1}$ and $642 \mathrm{~cm}^{-1}$ also showed shifts towards higher wavenumbers for osteoporotic nails. A study of 159 females participated with 34 cases of fractures, which was performed using an automated algorithm to detect the S-S content named the bone quality test (BQT) for each participant, which performed comparably with Dual-energy X-ray absorptiometry (DXA), the gold standard for diagnosing osteoporosis [73]. Furthermore, participants with history of fracture showed a significantly lower disulphide bond content than participants without history of fracture. The most recent study on this technique was conducted on 633 postmenopausal women to determine its performance against dual-energy X-ray absorptiometry T-score and QFracture, the former an $\mathrm{x}$-ray based technique and the latter a clinicial risk fracture questionnaire, both used to determine fracture risk [76]. The study proposed a novel algorithm, which combines Raman spectroscopy data with clinical data. The results showed that Raman spectroscopy can distinguish between subjects that sustained a fragility fracture and those who did not, with AUC of 0.74, which was higher than DXA (0.61) and QFracture (0.60). 


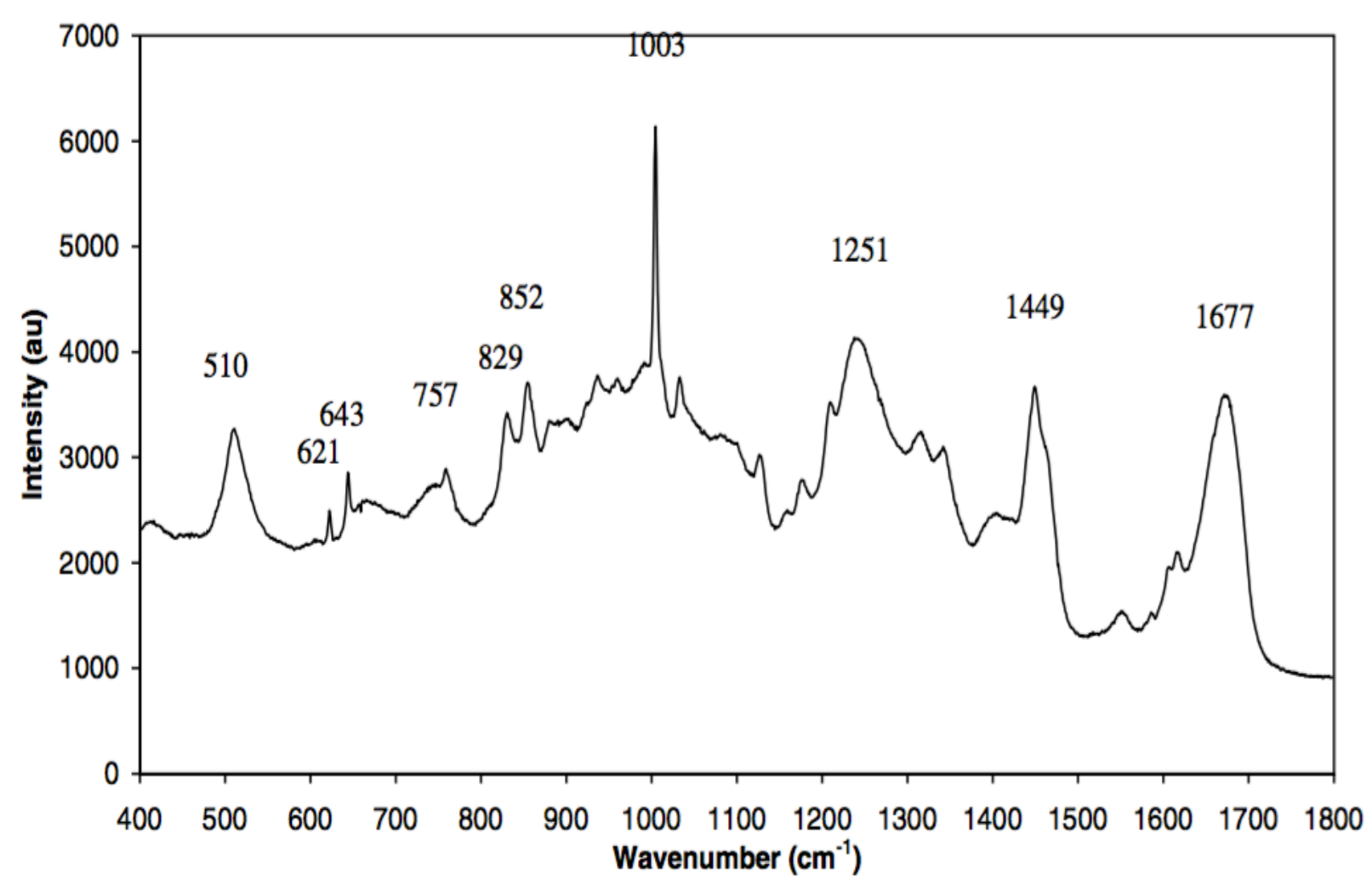

Figure 27. RS scan of a human nail for a spectral range of $300 \mathrm{~cm}-1$ to $1800 \mathrm{~cm}-1$ [74].

\subsection{RS of Human skin}

RS has been utilized in the last few decades to obtain chemical information for skin research. In-vivo and in-vitro studies have been conducted using RS, with one study using a confocal Raman microspectrometer for in-vitro sample analysis, and using a custom built housing with a inverted microscope for in-vivo measurements as shown in Figure 28 [77].

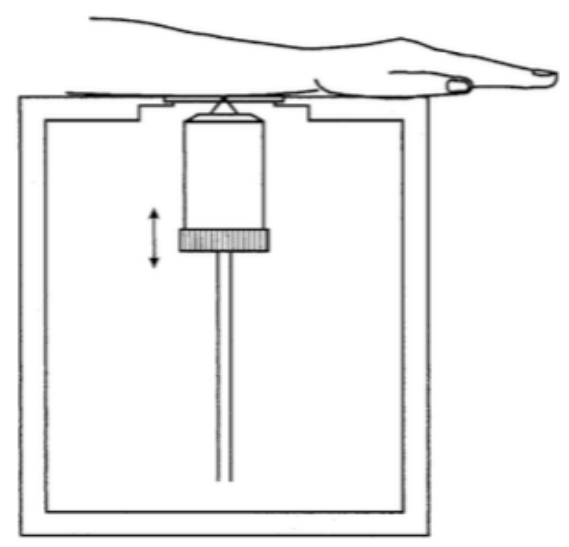

Figure 28. Custom -built inverted Raman microspectrometer housing for in-vivo Raman measurements of human skin [77]. 
The in-vitro results for the study showed RS scans for different layers of skin, starting from the outer most layer of the stratum corneum, followed by epidermis and then dermis, which is mostly composed of Human collagen Type I and III [77]. This was evidenced the by the similarity between the RS scans for dermis and human collagen, shown in Figure 29.

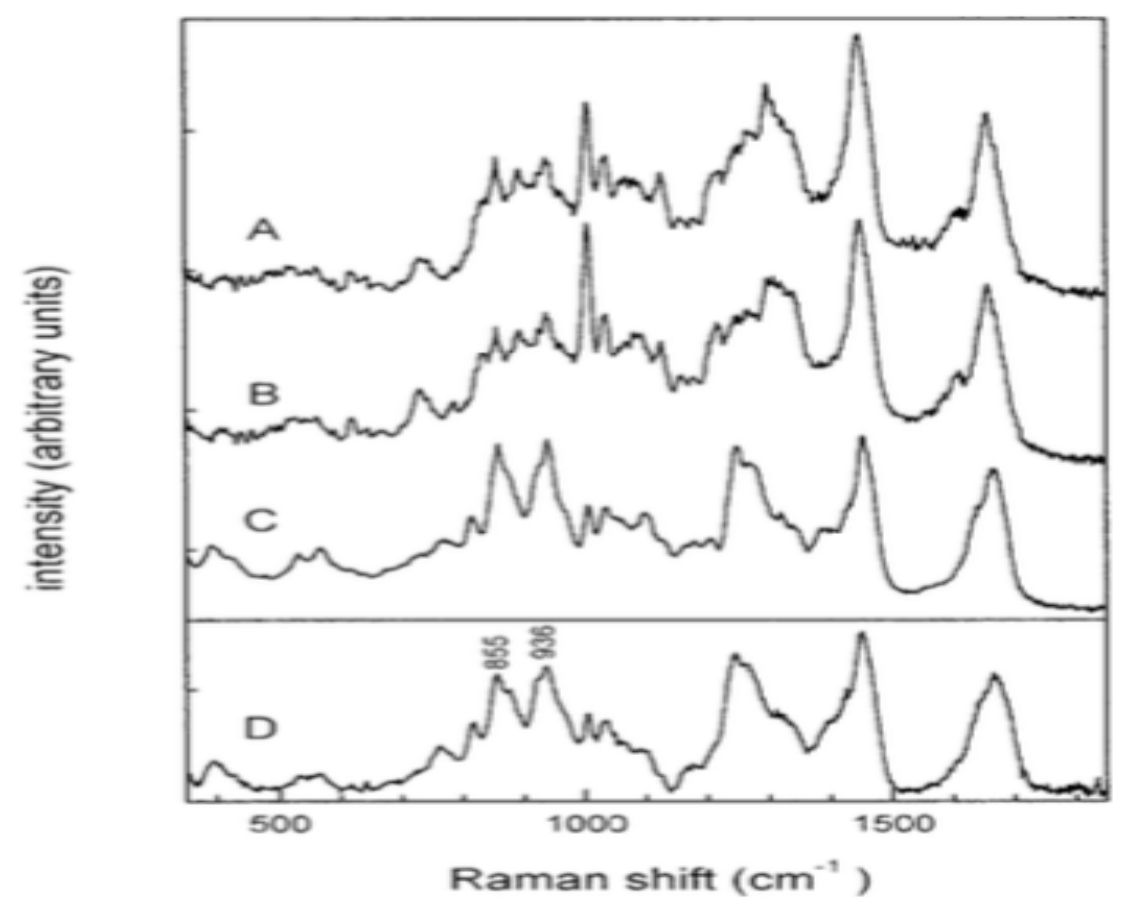

Figure 29. in-vitro Raman spectroscopy results for different layers of human skin, A is stratum corneum, B is epidermis, $C$ is dermis and D is human collagen I [77].

\subsubsection{Skin cancer diagnosis}

The possibility of using Raman micro-spectroscopy for real-time in vivo diagnosis of nonmelanoma skin cancer has been tested, using a portable confocal Raman system with a handheld probe. The results obtained from the study showed a $95 \%$ of classification accuracy [60]. In another preliminary study a real-time Raman spectroscope was used to distinguish between benign skin lesions and malignant melanoma (from benign pigmented lesions) [59]. The study measured 518 benign and malignant skin lesions from 453 patients. The results were compared against the gold standard histopathology. Three different diagnosis classifications were analyzed. The sensitivities for which ranged from 0.99 to 0.90 and the specificities ranged from 0.17 to 0.68 . Overall the study showed that RS gives good diagnostic accuracy in distinguishing normal from benign skin lesions. 


\subsection{Raman spectroscopy of MSU crystals}

RS has been previously used to access the chemical composition of cystine and uric-type Kidney stones [43]. Samples were acquired for the study, along with lab grade Monosodium urate (MSU) and uric acid. RS scans were obtained for all aforementioned specimens, with the goal to assess whether kidney stones matched more closely to uric acid or MSU. The results matched uric acid, but the Raman spectroscopic data for MSU was also first established in the study. The RS scan for MSU is given in Figure 30 and its chemical structure in Figure 31.

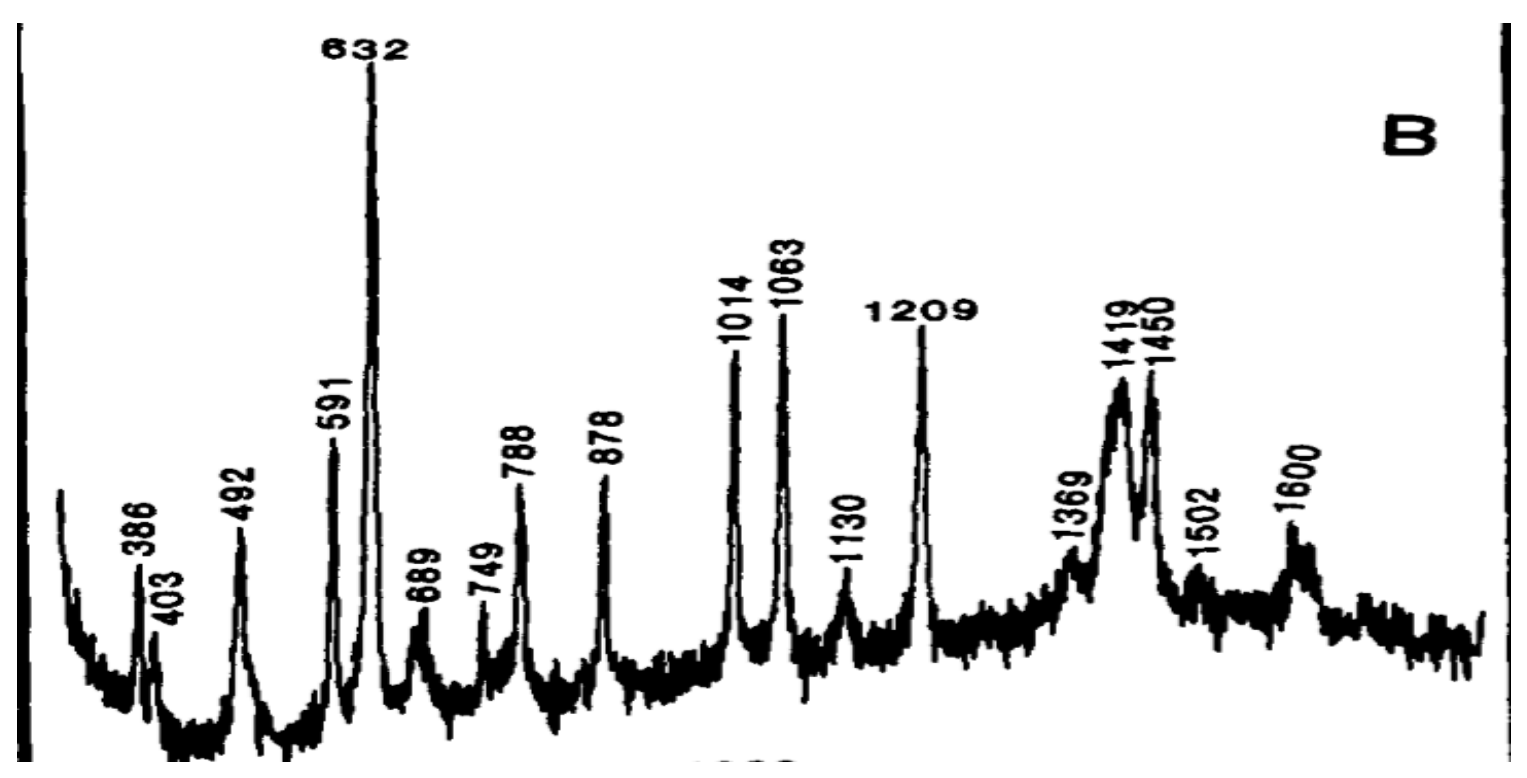

Figure 30. Raman spectrum of synthetic monosodium urate [43].

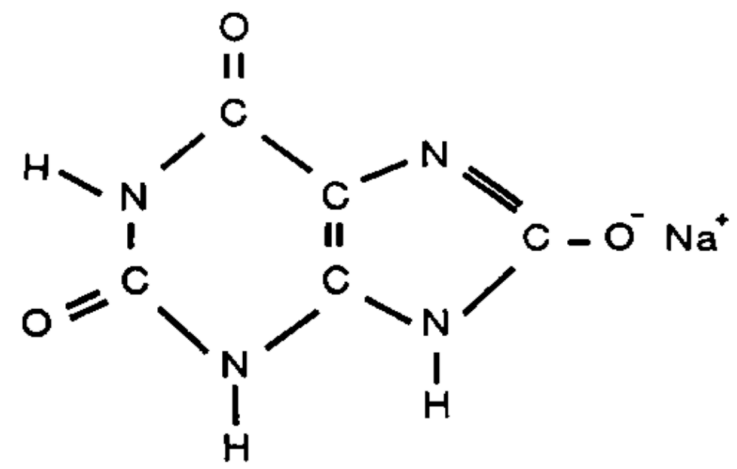

Figure 31. Chemical structure of monosodium urate (C5H3N4O3.Na) [43].

As shown in Figure 30, MSU has 18 Raman peaks. The peak assignments for these peaks were determined from literature and are shown in Table 2. However, assignments for some peaks could not be found in literature. 
Table 2. Peak assignments for MSU Raman bands.

\begin{tabular}{|l|l|l|}
\hline MSU peak cm $^{-I}$ & Peak assignment & Reference \\
\hline $\mathbf{3 8 6}$ & - & - \\
\hline 403 & - & - \\
\hline 492 & C-N-C vibrations & {$[43]$} \\
\hline $\mathbf{5 9 1}$ & C-N-C vibrations & {$[43]$} \\
\hline $\mathbf{6 3 2}$ & Purine ring vibration & {$[4]$} \\
\hline $\mathbf{6 8 6}$ & - & \\
\hline $\mathbf{7 4 9}$ & N-H bending vibrations & {$[43]$} \\
\hline $\mathbf{7 8 8}$ & - & - \\
\hline $\mathbf{8 7 8}$ & C-C stretching & {$[78]$} \\
\hline $\mathbf{1 0 1 4}$ & - & - \\
\hline $\mathbf{1 0 6 3}$ & C-C skeletal stretch & {$[78]$} \\
\hline $\mathbf{1 1 3 0}$ & C-C skeletal stretch & {$[78]$} \\
\hline $\mathbf{1 2 0 9}$ & C-N stretching & {$[78]$} \\
\hline $\mathbf{1 3 6 9}$ & - & - \\
\hline $\mathbf{1 4 1 9}$ & C=C stretching & {$[78]$} \\
\hline $\mathbf{1 4 5 0}$ & CH ${ }_{2}$ bending mode & {$[42]$} \\
\hline $\mathbf{1 5 0 2}$ & N-H bending & {$[78]$} \\
\hline $\mathbf{1 6 0 0}$ & C=O stretching & {$[78]$} \\
\hline & & \\
\hline & & \\
\hline
\end{tabular}

\subsection{RS of synovial fluid}

RS has been used previously to identify the presence of MSU crystals and Calcium pyrophosphate dihydrate crystal (CPPD), which is characteristic of Pseudogout, in synovial fluid extracted from patients. Pseudogout is a disease that can cause acute inflammatory arthritis, which occurs as a result of crystal deposition of CPPD crystals on joints [2]. These findings were compared to conventional analysis by Polarized light microscopy (PLM) [4], [31]. 35 synovial fluid samples were collected, ten of which were from clinically diagnosed gout sufferers [31]. The technique was able to identify seven of the ten diseased samples and overall 32 of the 35 samples were correctly identified by RS, which considered the presence of the $631 \mathrm{~cm}^{-1}$ peak, as confirmatory of gout. However, one of the diseased samples not detected by RS was later determined to be negative for MSU after reanalysis by RS; a false positive diagnosis by PLM. In addition, the technique identified all MSU positive samples and one additional positive was found, which on reanalysis with PLM, was revealed to be a positive. However, the technique 
required extensive sample preparation, including digestion of the fluid with lyophilized hyaluronidase powder for 15 mins in a glass centrifuge tube, followed by centrifugation, as synovial fluid contains organic debris. This greatly increased the diagnosis time for the technique. The study also showed that RS had a lower limit of detection than PLM, which can result in a higher sensitivity. RS scans for the data obtained in the study are shown in Figure 32.

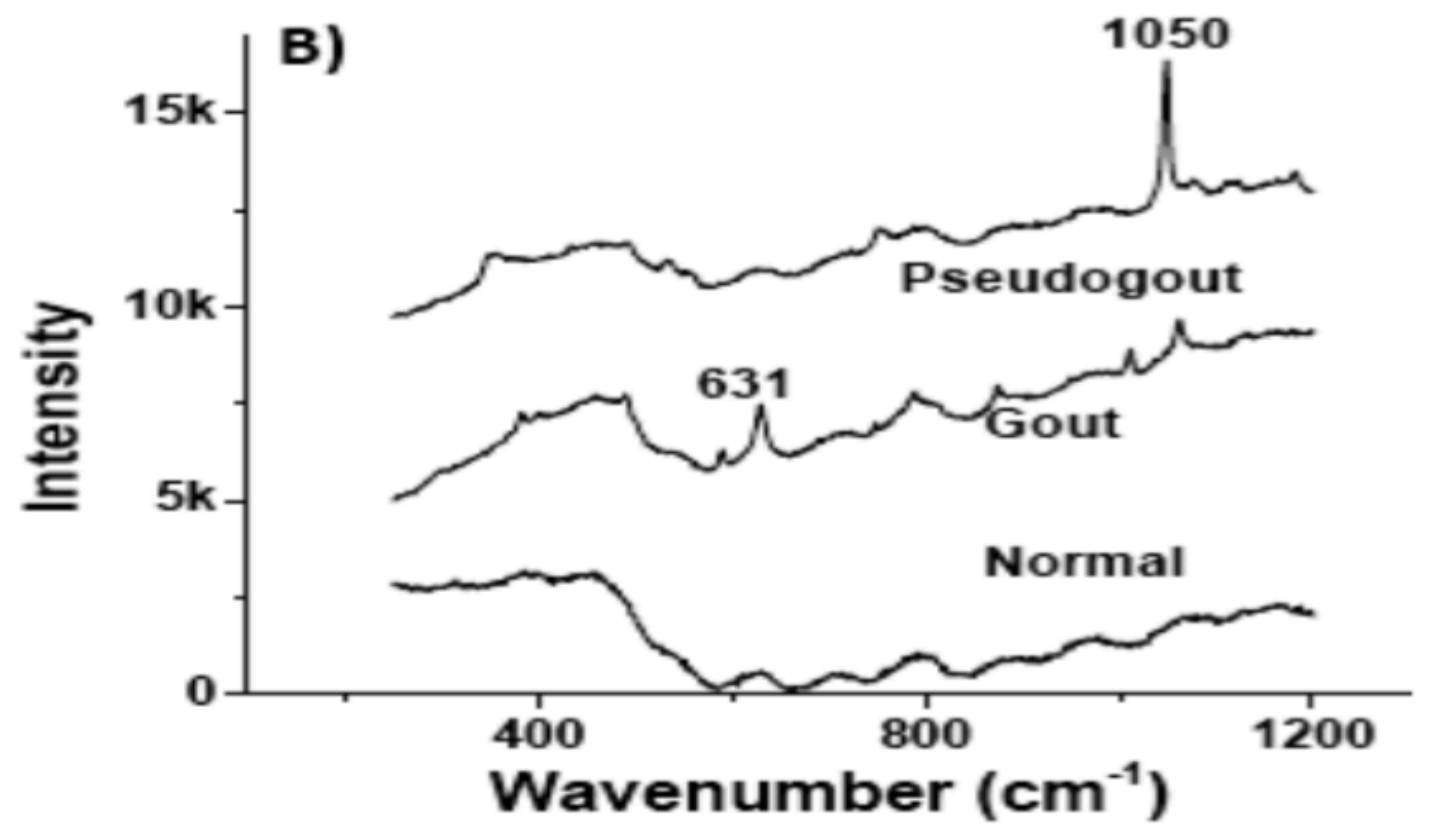

Figure 32. RS scan of synovial fluid after digestion and centrifugation for normal, gout and Pseudogout samples [31].

In a another study by the same group, a customized Raman spectrometer was developed for the same purpose but filtration was used instead of the centrifugation process [4]. This study only considered RS analysis of synthetic MSU and CPPD, which considered the presence of $590 \mathrm{~cm}^{-1}$ and $631 \mathrm{~cm}^{-1}$ peaks as confirmatory of MSU crystals. The results of the study showed that their RS device could detect MSU with a concentration as low as $0.1 \mu \mathrm{g} / \mathrm{mL}^{-1}$, which they concluded was a lower limit of detection then the clinically reported concentrations. A further study was conducted on the influence of background fluorescence in RS scans of synovial fluid [79]. Seven synovial fluid samples from patients with 'gout like symptoms' and three asymptomatic samples with no history of joint disease were collected. The study aimed to the see the relation between background fluorescence and excitation wavelength of the Raman spectroscope. The study showed that background fluorescence was higher in excitation wavelengths below $700 \mathrm{~nm}$. The study concludes that MSU crystals can clearly be observed in 
synovial fluid by RS at an excitation wavelength of $785 \mathrm{~nm}$. Furthermore, the study observed that symptomatic fluid samples had significantly lower background fluorescence intensity than asymptomatic samples. The major drawback of the technique is that it requires even more sample preparation then PLM and similar invasive extraction protocols as the aspirate fluid analysis by PLM. Thereby, it holds no potential to replace the gold standard since it will result in a higher cost and longer diagnostic times due to its extensive sample preparation. Furthermore, Using RS as an in-vitro tool is not the best use of its capabilities, as it lends it self to making invivo measurements and non-invasive diagnosis. The purpose of the research undertaken here is to evaluate the potential of Raman spectroscopy to detect MSU crystals non-invasively at the MTP joint. 
Chapter 5: Methods 


\subsection{Methodology}

The primary objective of this research is to investigate if RS of peripheral joints could be used for non-invasive detection of monosodium urate crystal deposits, which are characteristic of gout. In order to explore this, the presence of MSU deposition was evaluated at the first metatarsophalangeal joint (MTP) in two clinical trials conducted in the Rheumatology Department, Nottingham City Hospital, Nottingham, UK.

\subsubsection{Pilot study}

The first study, named the pilot study (Pilot), recruited 20 subjects, 10 with clinically diagnosed gout and 10 clinically diagnosed to have Osteoarthritis (OA). Validation technique used on gout patients was SF analysis of aspirated fluid. The scanning procedure and instrumentation was similar to a pilot study by Curran [80], in which the scans of the MTP for two subjects were compared, one gouty and the other non-gouty, which were recruited at the Rheumatology Clinic at St. Michael's Hospital, Toronto, Canada (REB\# 14-902).

\subsubsection{Sons of gout study}

The second study, named the Sons of Gout study (SOG), recruited 25 subjects; sons of clinically diagnosed gout sufferers, using US as the validation technique. SUA levels for all 25 subjects were also collected. Subjects were divided into two groups, one that showed signs of gout under US, the others that did not. However, none of the subjects were clinically diagnosed with gout, which means that they were treatment naive. Different instrumentation and scanning procedures were implemented in SOG to that of Pilot. Using a RS device with a fibre optic probe and different acquisition settings to that of the pilot study, to improve both the clinical utility of the Snowy Range Sierra model and the quality of scans subsequently obtained. The information collected during these trials was analyzed automatically. Automated analysis was conducted using an algorithm in Matlab ${ }^{\circledR}$ R2016a, which removes noise and fluorescence to perform peak detection and comparisons, against the MSU standard obtained from a RS study of kidney stones by Kodati [43]. 


\subsection{Study design}

The personnel at the University of Nottingham in Nottingham, UK, designed both clinical studies. The data was collected by the personnel at University of Nottingham, under the supervision of Dr. Abhishek Abhishek.

\subsubsection{Pilot study design}

This study was performed in partnership with the department of Academic Rheumatology, University of Nottingham in Nottingham, UK, with ethics approval (University of Nottingham Medical School Ethics Committee \#D11092014 SoM ROD 14081) and conducted under the clinical supervision of Dr. Abhishek Abhishek (Clinical Associate Professor of Rheumatology, Faculty of Medicine \& Health Sciences, University of Nottingham, Nottingham, UK). The Study aimed to form two groups: gouty subjects and Non-gouty Subjects, recruited by Dr. Abhishek. Eligible gouty subjects were those that met the ACR clinical classification criteria for gout, which includes the gold standard of needle aspiration analysis using PLM [81]. Eligible Nongouty subjects were those that did not meet the ACR classification criteria. All Non-gouty subjects that participated in this study were clinically diagnosed with Osteoarthritis. The serum urate levels (SUA) were also determined for each subject. The study was un-blinded for all 20 subjects before the sample analysis was completed and the findings published [1], which is shown in Appendix A: Published paper.

\subsubsection{SOG Study design}

This study was also performed in partnership with Academic Rheumatology under the supervision of Dr. Abhishek Abhishek. The Study aimed to form two groups: gouty subjects and non-gouty subjects, through the recruitment of volunteers that were sons of gout sufferers. Therefore, none of the subjects were clinically diagnosed for gout, but instead were monitored for signs of gout at a clinical visit, using ultrasound, SUA analysis and physical examination by the clinician. With ethical approval and informed consent, gouty and non-gouty subjects participated in this study. The study as a whole comprised of around 120 subjects but only the first 25 subjects are considered here due to time constraints. Initially, the US and SUA results for the first 10 subjects were unblinded before RS scans were analyzed, whereas the results for the remaining 15 subjects remained blind to the interpreter of the Raman scans. The first 10 subjects were used as a training set to determine the accuracy of predicting the remaining 15 subjects. 
However, the results were unblinded for all 25 subjects before the sample analysis was completed. During the clinical visit, US and RS were both performed on each of the subjects', left and right MTP joints. Eligible gouty subjects were the ones with signs of gout under US, while Non-gouty subjects were without any signs of gout under US. The gold standard for gout diagnosis of SF analysis under PLM was not utilized in this study due to ethics concerns around aspiration of control subjects. Instead, US (Toshiba Aplio SSA-770A machine and a multifrequency (7-12 MHz) transducer, Otawara-shi, Tochigi-ken, Japan) was utilized as a detection technique. US features of a double contour signal (DCS), Tophus, Hyperechoic deposits (HEDs) and effusion where documented for both MTP joints for each subject. DCS and Tophus being the features, which are most specific for gout. In addition, SUA levels were also measured for each subject.

Classification criteria for gouty subjects:

Subjects that were considered as positive conditions in the SOG study, were the ones that met at least one of the following criteria:

1. DCS observed

2. Tophus observed

3. DCS and tophus observed

\subsection{Instrumentation}

The two studies used the same Snowy Range Instruments (SnRI), Sierra model (Laramie, WY, USA), but with different hardware, specifications and acquisition settings. Pilot study used a portable benchtop Raman device, whereas SOG study used a fibre-optic probe model, which was a modified version of the instrument used for the pilot study.

\subsubsection{Snowy range Sierra model for pilot study}

RS was performed for the Pilot using a portable benchtop Raman device from Snowy Range Instruments (SnRI), Sierra model (Laramie, WY, USA) as shown in Figure 1. Prior to use, SnRI technicians' calibrated the system. Surface sampling was selected as the sampling position for the instrument. Additionally, the instrument was operated with specified settings using builtin software on a portable PC from SnRI and the recorded data was stored on the PC in spc file 
format. The instrument specifications are outlined in Table 1 and information on acquisition settings is provided in chapter 1.3.2 Acquisition settings.

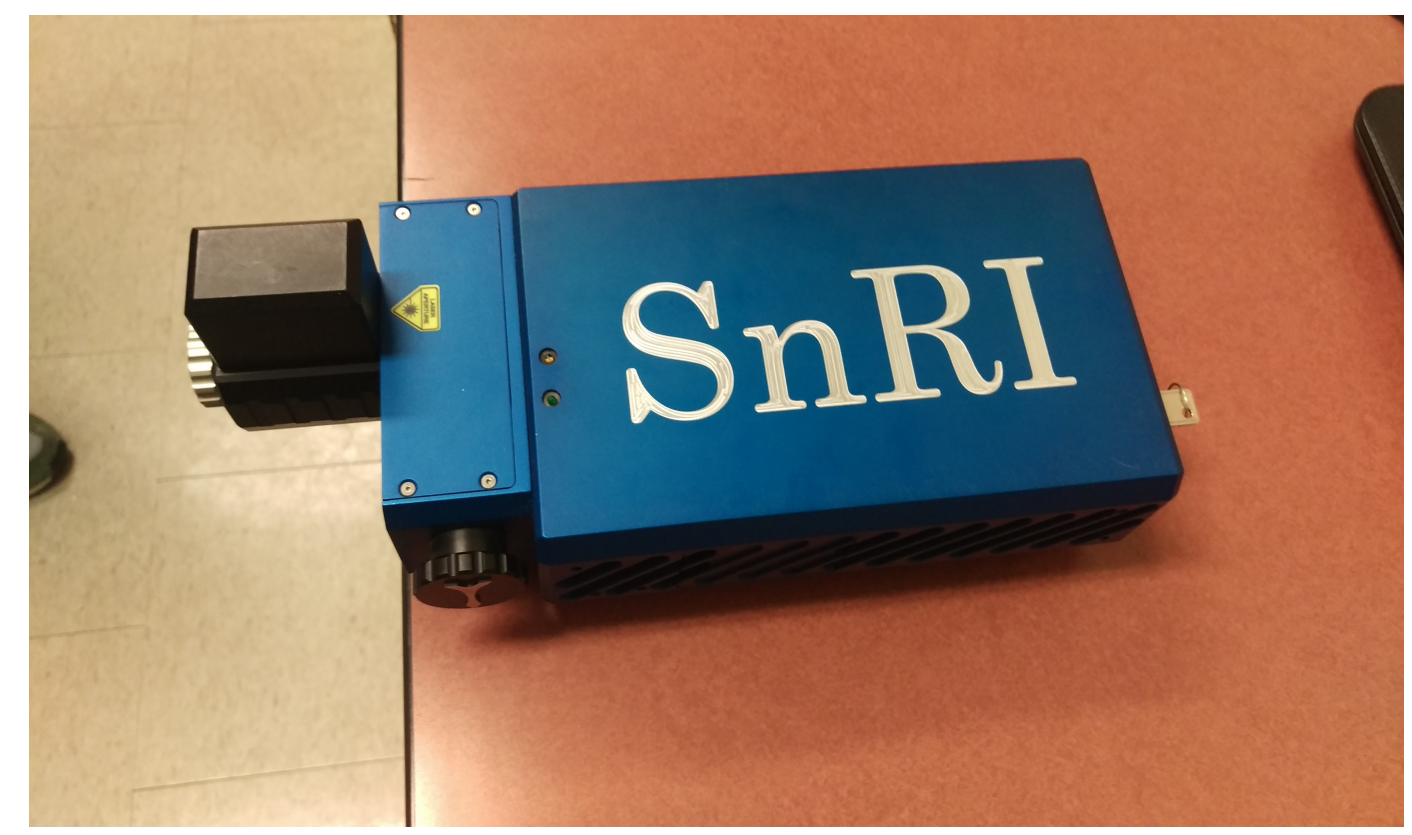

Figure 33. Top-view of SnRI-Sierra models Raman spectroscope used for the pilot study data collection.

Table 3. Pilot study instrument specifications.

\begin{tabular}{|c|c|}
\hline Parameter & Specification \\
\hline Laser & $785 \mathrm{~nm}$ diode laser \\
\hline Laser power & $<100 \mathrm{~mW}^{*}$ \\
\hline Spectral resolution & $4 \mathrm{~cm}^{-1}$ \\
\hline Spectral range & $200-2000 \mathrm{~cm}^{-1}$ \\
\hline Sampling mode & Small spot sampling* \\
\hline Focal length & $2.8 \mathrm{~cm}$ \\
\hline
\end{tabular}

* Adjustable

\subsubsection{Snowy Range Fibre probe model for the SOG study}

SOG was conducted using a modified version of the Snowy Range Raman used for Pilot. The major difference being the sampling technique, which was now a hand-held fibre optic probe with adjustable focal length, as can be seen in figure 39. This allowed more control over the scanning position, allowed the use of similar focal length for each scan and minimized the effects of patient movement on the quality of RS scans. A $25 \mathrm{~mm}$ lens was used as the lens for 
the fibre optic probe. Prior to use, SnRI technicians' calibrated the system. Furthermore, for the instruments' adjustable focal length, standoff position 1 was used as shown in Figure 35.

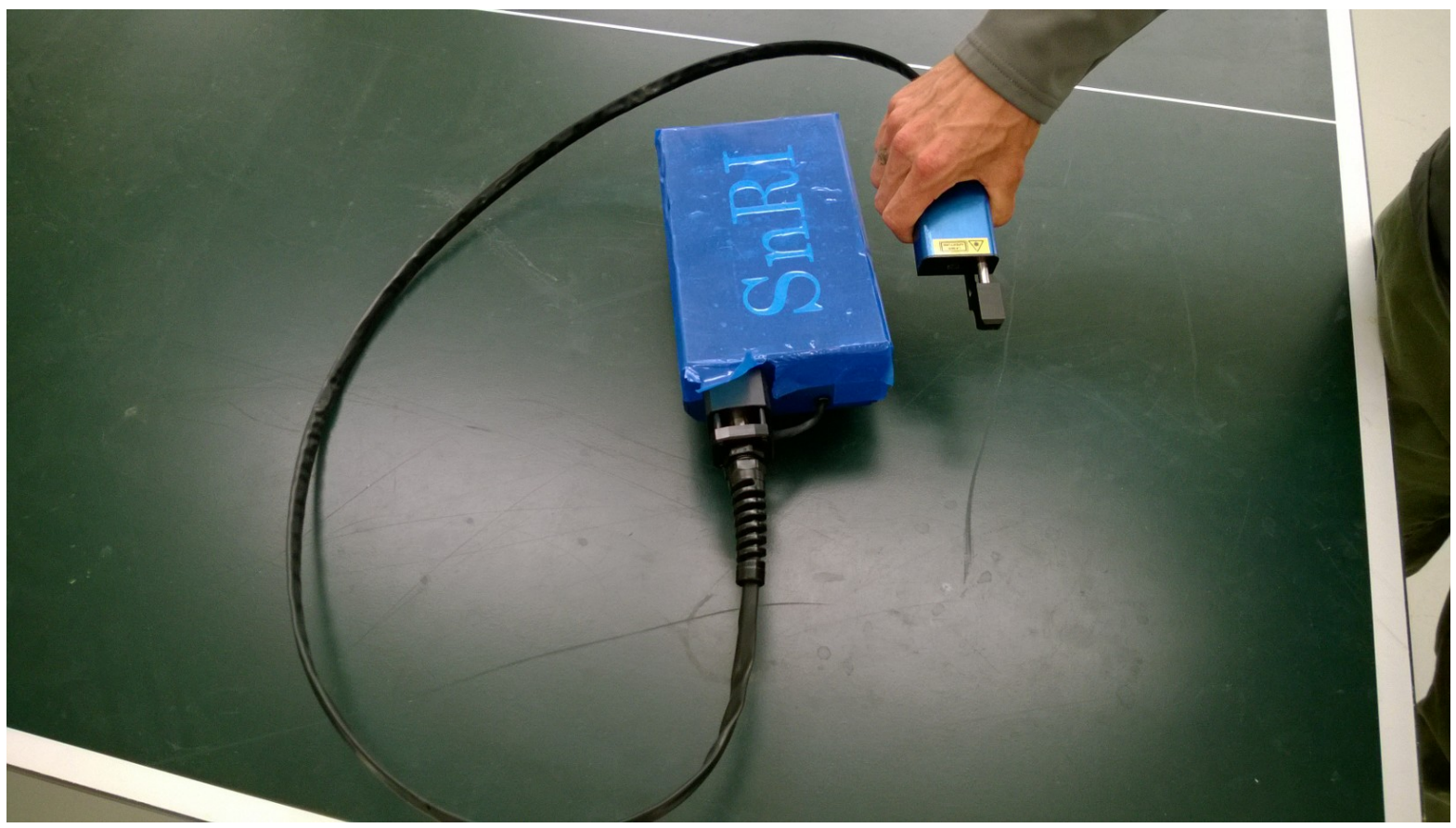

Figure 34. SnRI-Sierra Fiber probe model Raman spectroscope for SOG study data collection.

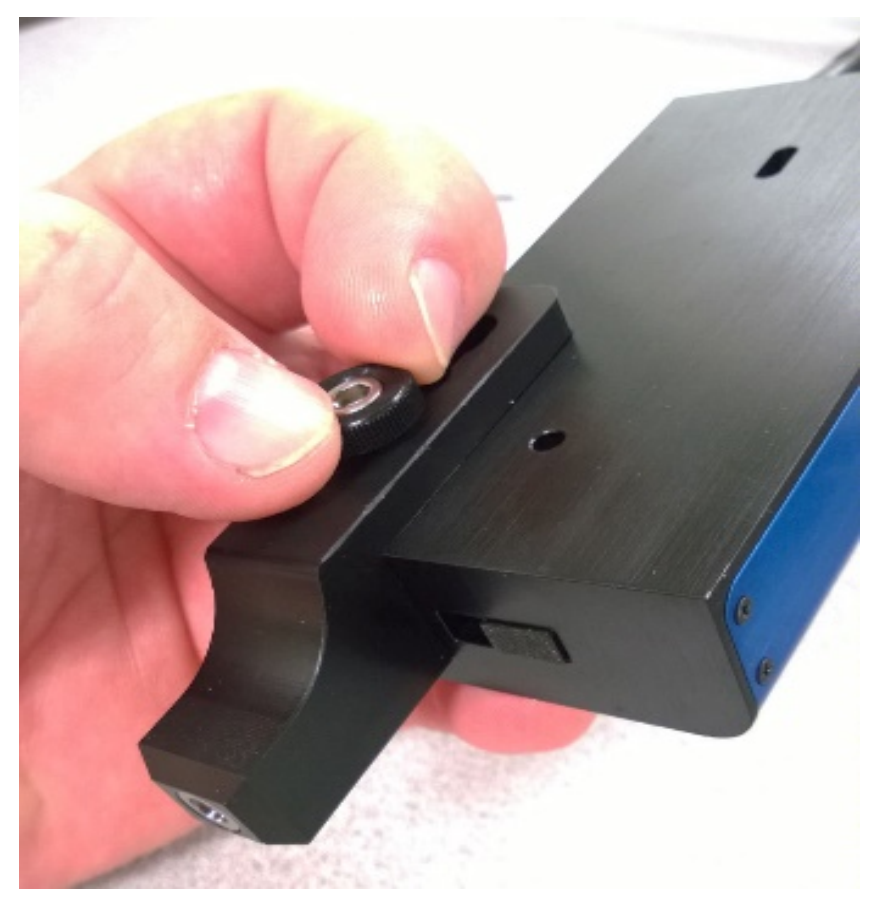

Figure 35. SOG-probe focal length adjusted to standoff position one to acquire RS scans for SOG study. 
The data was stored with the same process as for the pilot study RS. The instrument specifications are outlined in Table 1 and information on acquisition settings is provided in 5.5.2 Acquisition setting for SOG study

Table 4. SOG study instrument specifications.

\begin{tabular}{|c|c|}
\hline Parameter & Specification \\
\hline Laser & $785 \mathrm{~nm}$ diode laser \\
\hline Laser power & $<400 \mathrm{~mW}^{*}$ \\
\hline Spectral resolution & $4 \mathrm{~cm}^{-1}$ \\
\hline Spectral range & $200-2000 \mathrm{~cm}^{-1}$ \\
\hline Sampling mode & Small spot sampling* \\
\hline Focal length & $25 \mathrm{~mm}^{*}$ \\
\hline
\end{tabular}

* Adjustable (Refer to Appendix)

\subsection{Trial procedure}

\subsubsection{Pilot study}

The general method for positioning the device and the sampling point (MTP joint) was not only dependent upon the comfort of the patient but also on the limitations enforced due to the design of the RS device. In essence, the main requirements were such that the RS device should remain stationary provided that it is not hand-held and not in contact with the patient. Furthermore, the laser outlet was approximately kept at focal length, which ultimately ensured that the device never came in contact with the patient, so as to provide the best possible situation to acquire the relevant data and to also ensure that patient remained comfortable.

For the MTP joint, the patient was asked to remain seated on a chair with their knees bent slightly more than 90 degrees to the surface, as this would help avoid erratic movements during the test. However, the location of the laser outlet on the device prompted the need to raise the height of the patients' feet. Consequently, by placing an object of appropriate height under the feet, raised the joint to the same level as the laser outlet, as can be seen in an illustration of this setup shown in Figure 36. Furthermore, RS was performed in situ, thereby no sample preparation was required other than ensuring the laser outlet was positioned in the correct spot. 
As a result, this spot on the joint would be selected with the guidance of clinicians that were supervising the trials.

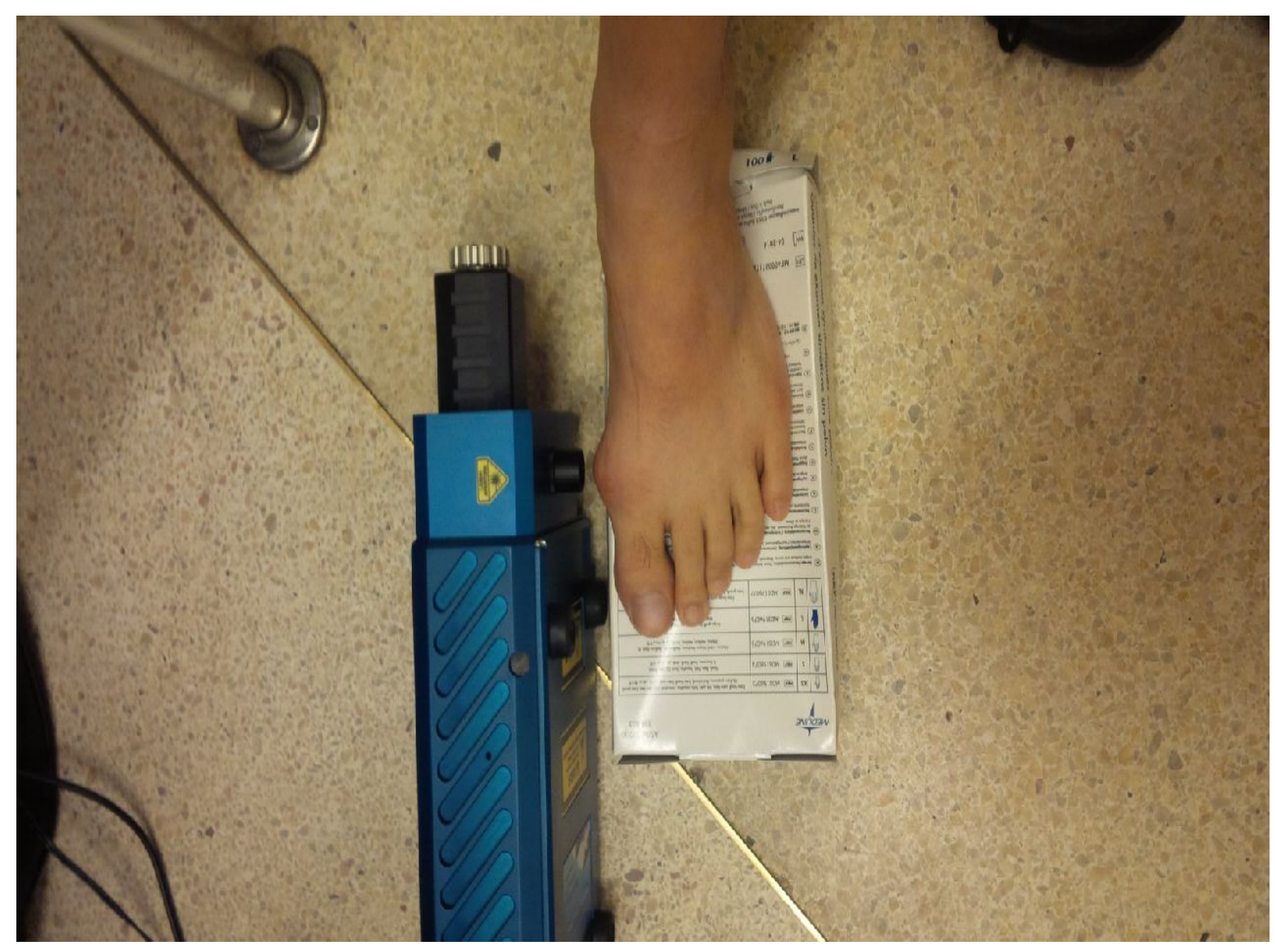

Figure 36. Trial setup for the pilot study, showing the Raman spectroscope positioned at the MTP joint.

\subsubsection{Trial procedure for SOG}

The general method for positioning the device and the sampling point (the patient's joints) was determined by the clinicians conducting the study in Nottingham. Three positions on the MTP joint were selected to conduct the scans for each foot, therefore a total of 6 scans per patient were obtained. These positions are indicated by red circles in Figure 37. Care was taken to avoid any unwanted movement of the handheld probe while acquiring the scan at these aforementioned positions. 


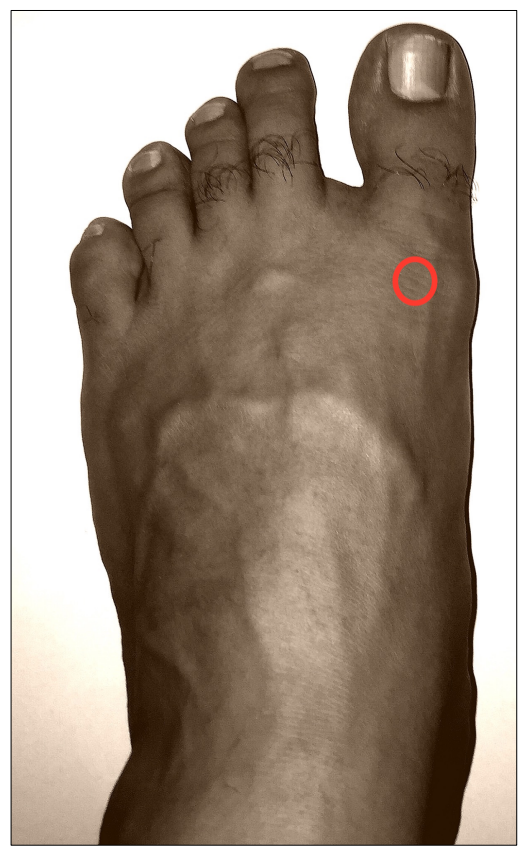

(a)

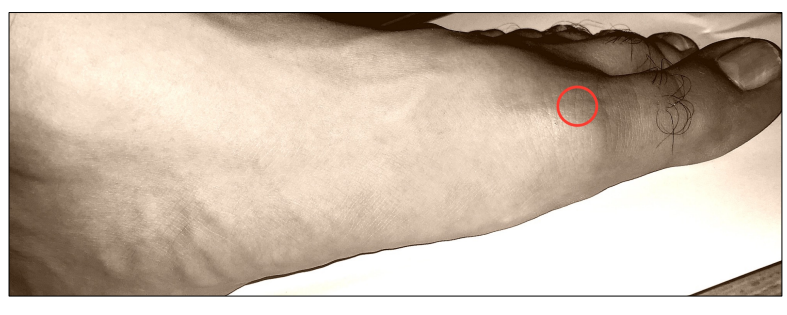

(b)

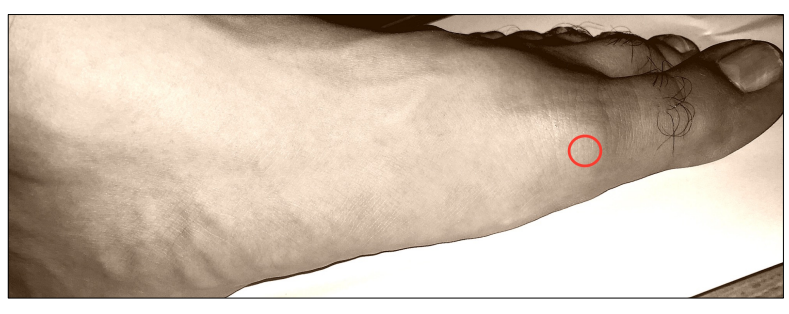

(c)

Figure 37. SOG study scanning positions: (a) Position 1, (b) Position 2 and (c) Position 3.

The positioning of the patient remained similar to the pilot study. However, there was no need for adjusting foot height to the laser outlet, since the new fibre device was fitted with a probe, which allowed more flexibility and control over acquiring scans. Scanning positions were increased to three positions rather than one as with Pilot, to increase the chance of detecting MSU. The fibre probe was equipped with a safety interlock button, which needed to be held down while the scanning was taking place. To initiate scanning, the Acquire command in the included acquisition software was pressed, in concurrence with the interlock button. Similarly to Pilot, no particular sample preparation was required other than ensuring the probe outlet was positioned in the correct spot. 


\subsection{Acquisition settings}

\subsubsection{Acquisition settings for Pilot}

The acquisition settings were adjustable using the built-in software provided with the Raman spectrometer. Thus, the parameters and their chosen settings are shown in Table 5.

Table 5. Pilot study SnRI Raman acquisition settings.

\begin{tabular}{|c|c|}
\hline Parameter & Setting \\
\hline Laser power & $72 \mathrm{~mW}$ \\
\hline Integration time & $10 \mathrm{~s}$ \\
\hline No of Acquisitions & 5 \\
\hline Referencing Mode & Reference every acquisition \\
\hline Sampling mode & Small spot sampling \\
\hline Laser outlet position & Surface sampling \\
\hline
\end{tabular}

As a result of the setting mentioned above, five acquisitions (scans) were obtained, and then were averaged to produce a single scan. Ultimately, this averaged scan was exported to the desired location on disk in a format known as $s p c$ that is reserved specifically for storing spectroscopic data. In general, the referencing mode closes the laser shutter and takes a scan of the inner optics before every acquisition. Then this reference is subtracted from the recorded scan for each acquisition.

\subsubsection{Acquisition setting for SOG study}

The acquisition settings were adjustable using the built-in software provided with the Raman spectrometer. Laser power was increased $(72 \mathrm{~mW}$ to $100 \mathrm{~mW})$ in comparison to the pilot study, as well as the integration time (10s to $20 \mathrm{~s}$ ), which were done to improve the signal-tonoise ratio. Thus, the parameters and their chosen settings are shown in Table 6.

Table 6. SnRI Fibre probe Raman acquisition settings for SOG study.

\begin{tabular}{|c|c|}
\hline Parameter & Setting \\
\hline Laser power & $100 \mathrm{~mW}$ \\
\hline Integration time & $20 \mathrm{~s}$ \\
\hline No of Acquisitions & 5 \\
\hline Referencing Mode & Reference every acquisition \\
\hline Sampling mode & Fibre optic probe \\
\hline Focal length & $25 \mathrm{~mm}$ \\
\hline
\end{tabular}


As with the pilot study, the five acquisitions were averaged to produce a single scan. Export of scans and the functioning of the referencing mode was the same as in the pilot study.

\subsection{Matlab routine for Determining MSU presence Automatically (both studies)}

The Matlab program SolasDX.m deals with processing of Raman spectral data taken with the RS device. The program functions similarly to determining MSU presence manually but was automated and coupled with routines for removal of noise and background fluorescence. Firstly, as a result of the automation, the analysis time will reduce and the accuracy of the results would increase. Secondly, reducing the noise and fluorescence will help extract the Raman scatter more conveniently. An algorithm was followed to ensure that the goal of the program is understood and achieved properly. As can be seen, the aforementioned algorithm is illustrated in Figure 38 and was implemented sequentially.

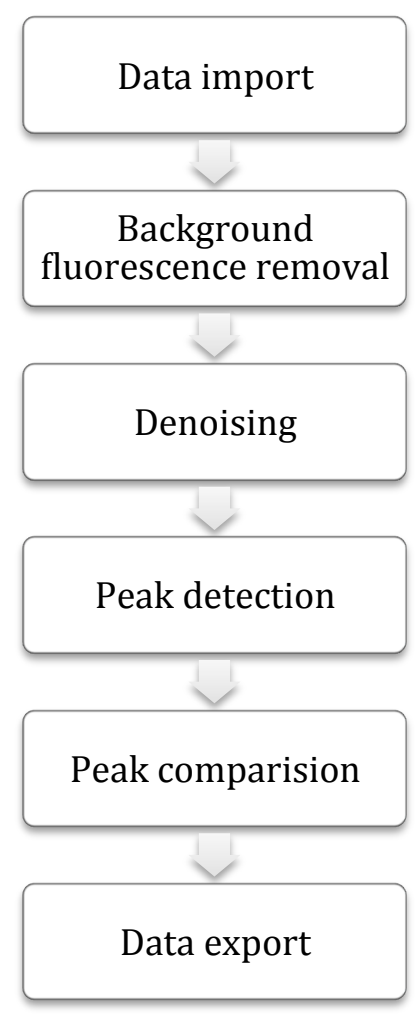

Figure 38 Raman processing algorithm for spectral processing for both studies

To begin with, data was imported using a built-in Matlab function tgspcread. As a result the Raman spectral data was imported, and their wavenumbers and intensities were stored in 
their individual 1-D matrixes. Thereafter, these imported spectrums were background corrected in order to remove the fluorescence, by using the function airpls [82]. The outputs from the previous function were passed through wavelet de-noising, by using the built-in Matlab function cmdnoise.m. Next, these de-noised spectrums were put through a built-in Matlab function for peak detection called mspeaks. $m$ to reveal major peaks. Then the detected peaks in the Raman scan were compared to the MSU standard peak locations determined from a Raman spectroscopy study conducted on uric-acid based kidney stones by Kodati[43], as shown in Figure 39. Finally, the program ends by exporting the desired outputs into a spreadsheet. In the following chapters, brief descriptions will be given on the functions used but the complete Matlab code is provided in Appendix B: Matlab code.

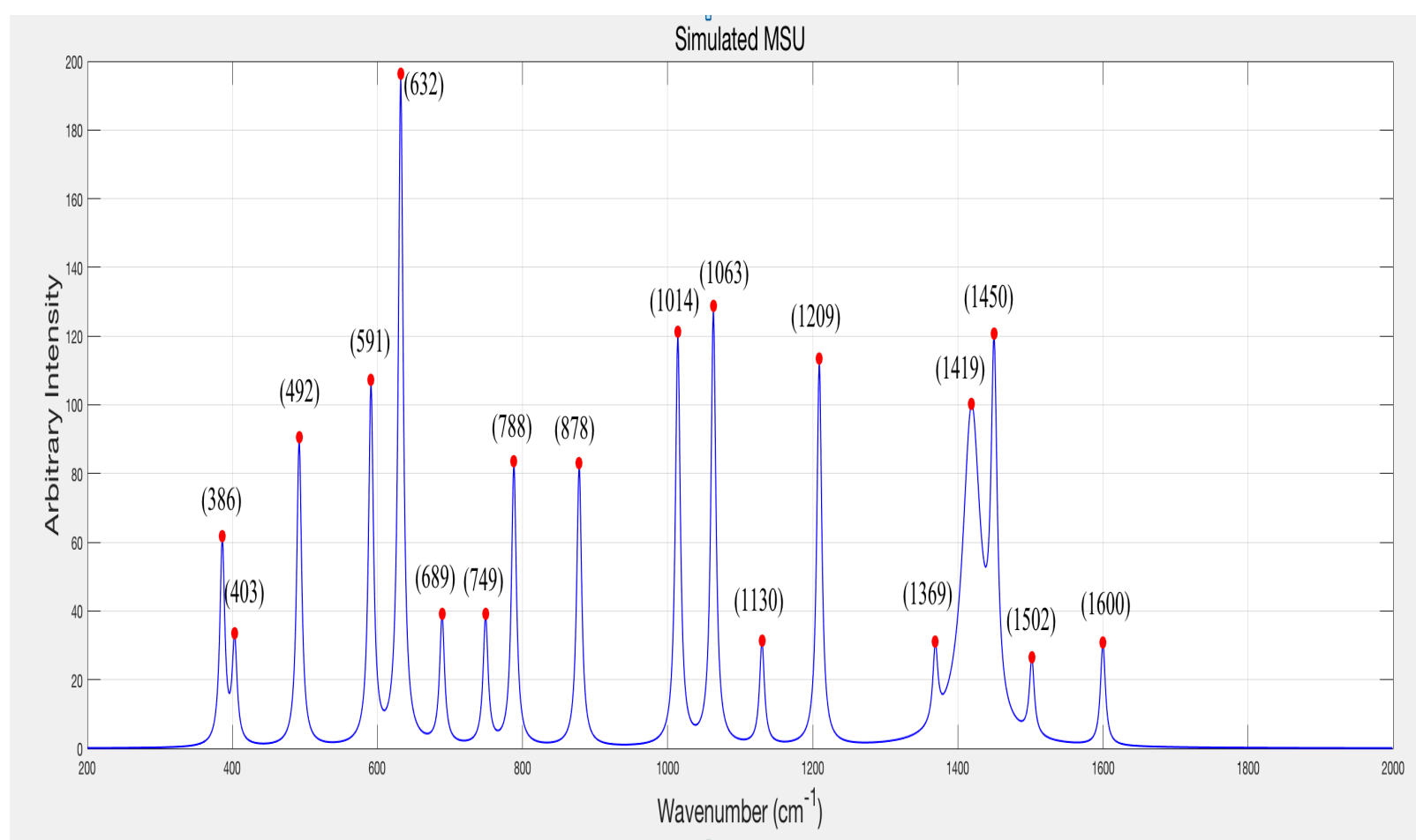

Figure 39. Simulated MSU with labelled Raman peak wavenumbers, recreated from [43].

\subsubsection{Data import}

Simple lines of code utilizing built-in Matlab funtions were implemented to import the required spectral data, for which the required input were the file names in the string format for all RS scans which were stored in appropriately named character matrixes. In addition, the wavenumbers and intensities for the input file were stored in separate matrixes. 


\subsubsection{1-Syntax for data import}

The following syntax was used to execute the function:

SPCStructGout $=\operatorname{tgspcread}($ Gouty $)$;

WavenumberGout $=$ SPCStructGout.X;

IntensityGout $=$ SPCStructGout.Y;

In this function, tgspcread, a built-in Matlab function were used to open the SPC file and create a 2-dimensional matrix structure of the data that contains the wavenumber and intensity from the SPC file. Then this data was taken from this SPC structure and divided into two 1Dimensional matrixes named after their contents. For clarification the wavenumbers and intensities are inserted into variables with suitable names.

\subsubsection{Background florescence removal}

The function airpls.m was executed for background florescence removal, known as the Adaptive iterative reweighted penalized least squares (airPLS) [82]. To begin with, the function determines a baseline fit by finding the baseline points associated with the spectrum and creating a polynomial fit through these base points. Then this polynomial fit was subtracted from the intensities of the spectrum. As a result, the output was a corrected spectrum with a flatter baseline, thereby removing the florescence. To demonstrate, a test of the function was conducted as shown in Figure 40, where a simulated spectrum with a curved baseline, background corrected result, and the baseline fit is shown.

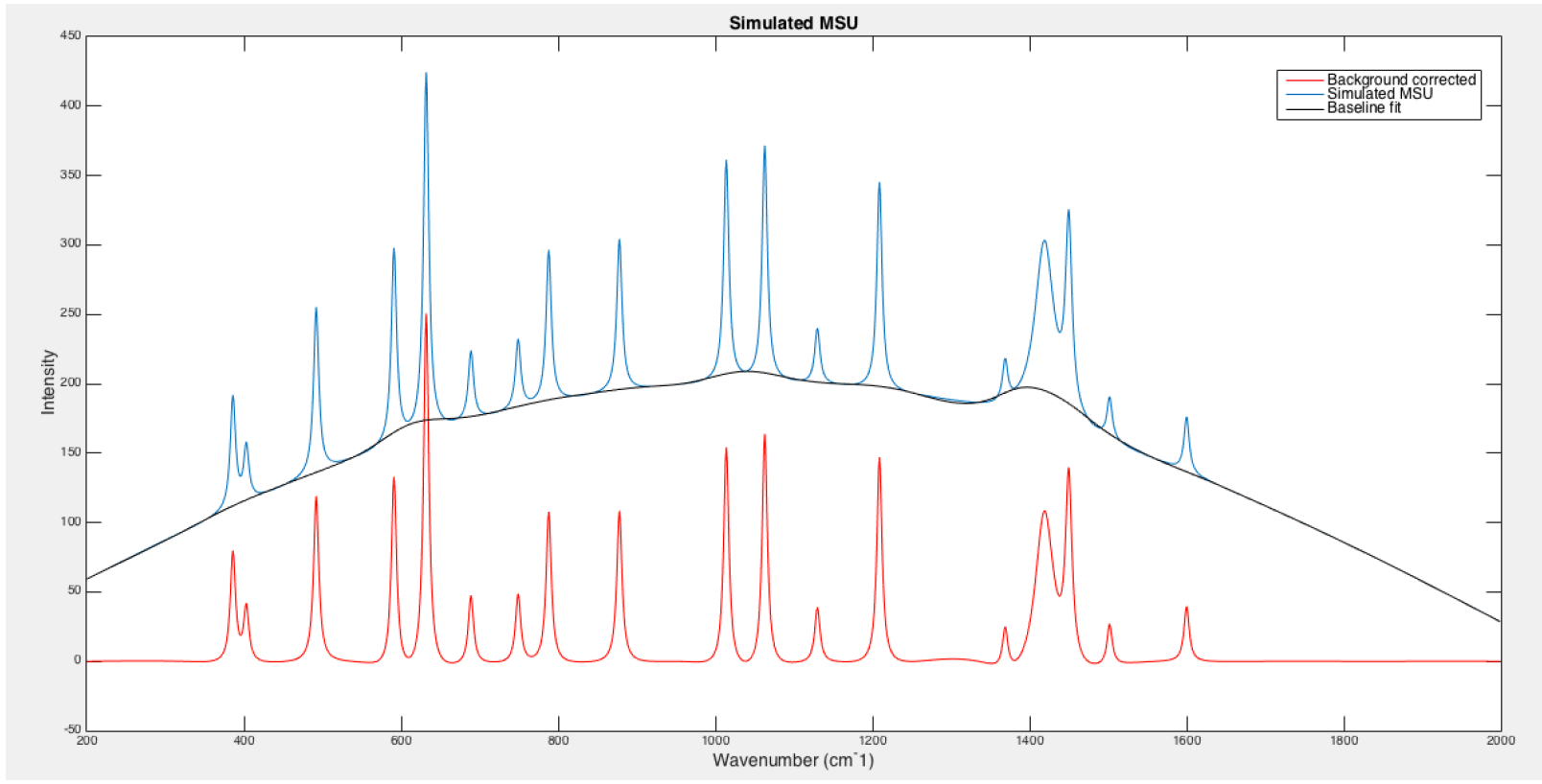

Figure 40. Background removal demo, containing the simulated MSU, corrected MSU and baseline fit plots. 
Furthermore, the function was applied for all RS scans. Additionally, the descriptions of the inputs and outputs for the function are given in Table 7. Further description of the inputs is given in the function's m-file airPLS.m in Appendix B: Matlab code.

\subsubsection{Syntax for background fluorescence removal}

The following syntax was used to execute the function:

function [AdjustedIntensityPLS,fittedvector]= airPLS(X,lambda,order,wep,p,itermax)

Table 7. Variable descriptions for airPLS function.

\begin{tabular}{|c|c|}
\hline Variable & Description \\
\hline$X$ & Row matrix of spectrum intensity (size $m^{*} n, m$ is sample and $n$ is variable) \\
\hline lambda & $\begin{array}{l}\text { Lambda is an adjustable parameter that controls the fitting function. The larger lambda is, } \\
\text { smoother the fitted vector and was set to } 40\end{array}$ \\
\hline order** & An integer indicating the order of the difference of penalties, which was set to 1 \\
\hline wep $* *$ & Weight exception proportion at both the start and end, which was set to 0.075 \\
\hline$p * *$ & Asymmetry parameter for the start and end that was set to 0.1 \\
\hline itermax** & Maximum number of iterations was 6 \\
\hline AdjustedIntensityPLS* & The corrected spectra intensity matrix ( size $\left.m^{*} n\right)$ \\
\hline fittedvector* & The fitted vector (size $\left.m^{*} n\right)$ \\
\hline
\end{tabular}

*Output

** Set to default values

\subsubsection{De-noising}

The function cmddenoise. $m$ for performing wavelet analysis was used to reduce the noise in the RS scans. To begin with, the function takes a row matrix of the background corrected intensity from the preceding function and then performs multilevel-1D wavelet decomposition. As a result, the signal was divided into two halves, low-frequency components and high frequency components. A thresholding expression was then used on the high frequency components to remove noise, essentially acting as a filter. Finally, the signal was reconstructed by using multilevel-1D wavelet reconstruction. Consequently, this reconstructed signal was the de-noised version of the input signal. In addition, the descriptions of the inputs and outputs for the function are given in Table 8. 


\subsubsection{Syntax for wavelet de-noising}

The following syntax was used to execute the function:

\section{function [denoised_intensity] = cmddenoise(sig,wname,Level,sorh);}

Table 8. Variable descriptions for wavelet_denosing function.

\begin{tabular}{|l|l|}
\hline \multicolumn{1}{|c|}{ Variable } & \multicolumn{1}{c|}{ Description } \\
\hline sig & $\begin{array}{l}\text { Row matrix of background corrected spectra from previous function (size } \mathrm{m} * \mathrm{n}, \mathrm{m} \text { is sample } \\
\text { and } \mathrm{n} \text { is variable) }\end{array}$ \\
\hline level & The level used for multilevel-1D wavelet decomposition, was set to 2 \\
\hline sorh & $\begin{array}{l}\text { Threshold rule that has three options: 's' and ' } \mathrm{h} \text { ', which are soft and hard thresholding, was set } \\
\text { to ' } \mathrm{s} \text { ' }\end{array}$ \\
\hline wname & Wavelet used for wavelet analysis, was set to 'bior3.5' \\
\hline denoised_intensity* & The de-noised spectra intensity matrix (size $\mathrm{m} * \mathrm{n})$ \\
\hline
\end{tabular}

*Output

Furthermore, Increasing level results in a smoother result that preserves the width and shape of the original peaks. The selection of level was made after observing the affects of different level values on scans. A level of 2 gave the most appropriate extent of denoising without significant loss of data. It is to be noted that the selection of the wavelet depends on the peak shape for the signal being de-noised. To demonstrate, a test of the function is shown in Figure 41 that includes: the simulated spectrum with random noise, simulated spectrum without noise and the de-noised version. For the case at hand, a wavelet from the biorthogonal wavelet family, specifically, "bior3.5" was used to perform the test, as the peak shape for the reconstructed spectrum was found to be fairly similar to the original signal.

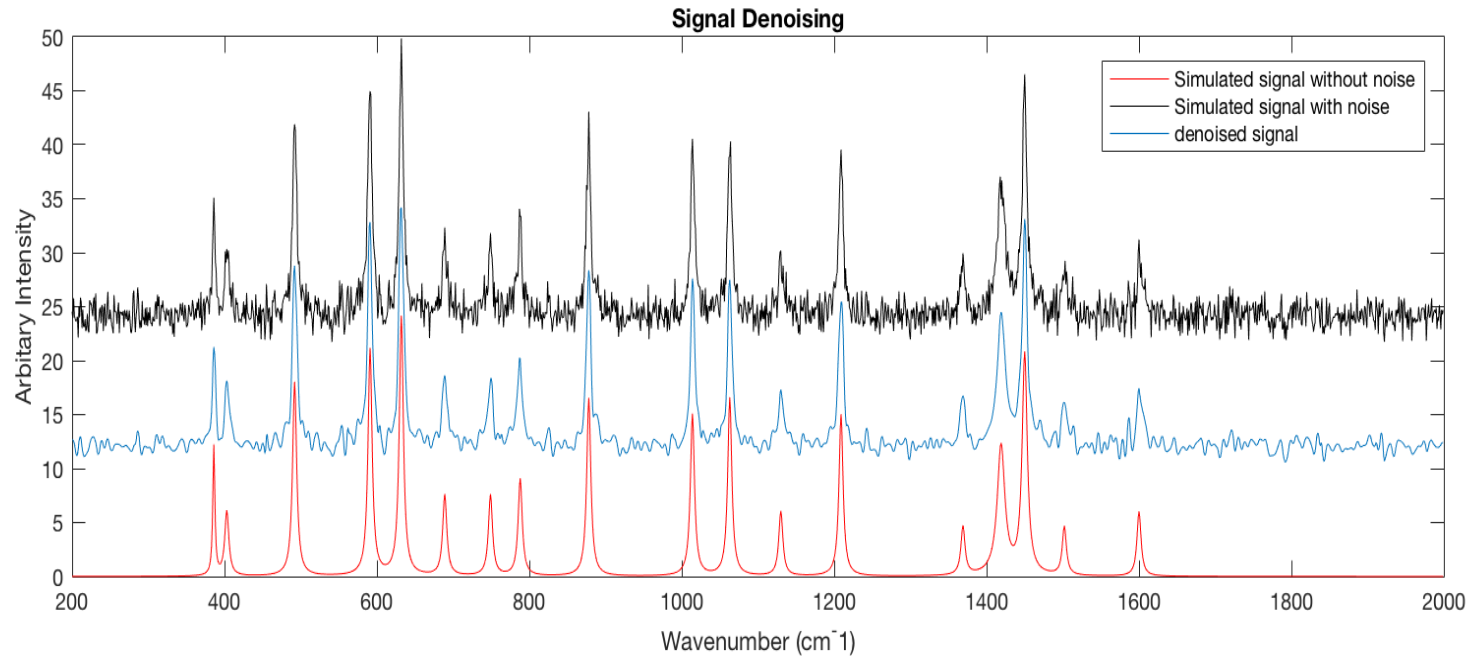

Figure 41. De-noising demo, containing the simulated signal with noise, without noise and the de-noised signal. 


\subsubsection{Peak detection}

The built-in Matlab function mspeaks from the bioinfomatics toolbox was utilized to determine peak locations for the de-noised scans from the preceding function. Furthermore, Multiple input options are available for mspeaks that were mainly focused on filtering properties. In this instance, the properties used will be Height filtration and Full-width at half height (FWHH) filtration. First, the function began by determining all peaks. After, which the selected filtration properties were executed, Height filtration removes the peaks from the output matrix that are below the value set for minimum permissible height. Similarly, the FWHH filtration removes the peaks from the output matrix that are below the value set for minimum permissible full-width at half height. In addition, the descriptions of the inputs and outputs for the function are given in Table 9.

\subsubsection{Syntax for peak detection}

The following syntax was used to execute the function:

peaklist= mspeaks(X,Y,'HeightFilter',HeightFilterValue,'FWHHFilter', FWHHvalue);

Table 9. Variable descriptions for mspeaks function.

\begin{tabular}{|c|c|}
\hline Variable & Description \\
\hline $\boldsymbol{X}$ & Column matrix of Wavenumber values ( \\
\hline $\boldsymbol{Y}$ & $\begin{array}{l}\text { Column matrix of denoised spectra from previous function (size } m^{*} n, n \text { is sample and } m \text { is } \\
\text { variable) }\end{array}$ \\
\hline 'HeightFilter' & To enable Heightfilter property \\
\hline HeightFiltervalue & $\begin{array}{l}\text { Value set for Heightfilter will not output peaks below this value of Height. Permissible } \\
\text { value of }>0 \text {, the value was set to } 6\end{array}$ \\
\hline 'FWHHFilter' & To enable Full-Width-Half-Height (FWHH) property \\
\hline FWHHvalue & $\begin{array}{l}\text { Value set for FWHHFilter that will not output peaks below this value of FWHH. } \\
\text { Permissible value of }>0 \text {, the value was set to } 2\end{array}$ \\
\hline peaklist* & Column matrix of peak locations and their corresponding height (size varies) \\
\hline
\end{tabular}

*Output

\subsubsection{Peak comparison}

This piece code conducts a comparison between the peaks found in the gouty patient from the preceding function and MSU standard provided that the allowed shift from MSU standard was $\pm 4 \mathrm{~cm}^{-1}$ wavenumbers (as this is the resolution of the RS device). In other words, a peak from the MSU standard was present in the gouty patient if its location is within $\pm 4 \mathrm{~cm}^{-1}$ 
from one of the peak locations for MSU standard. The descriptions of the inputs and outputs for the function are given in Table 10.

Table 10. Variable descriptions for peak comparison function.

\begin{tabular}{|l|l|}
\hline \multicolumn{1}{|c|}{ Variable } & \multicolumn{1}{c|}{ Description } \\
\hline $\boldsymbol{U}$ & Peaklist from previous function is stored in this row matrix \\
\hline peaklist_S & A row matrix of size $18 \mathrm{x} 1$. Consists of the Peaklist for the MSU standard \\
\hline Upperlimit & $\begin{array}{l}\text { A Row matrix of zeroes is created, the size of which is: rows of U x rows of peaklist_S. The } \\
\text { number of rows will change but the number of columns will remain as 18 } 18\end{array}$ \\
\hline Lowerlimit & $\begin{array}{l}\text { A Row matrix of size } 18 \mathrm{x} 1, \text { where four is added to each row. This will be used to allow a } \\
\text { shift of }+4 \mathrm{~cm}^{-1} \text { from the MSU standard peaks } \\
\text { allow a shift of }-4 \mathrm{~cm}^{-1} \text { from the MSU standard peaks }\end{array}$ \\
\hline ind and $\boldsymbol{k}$ & Counters for the loops \\
\hline
\end{tabular}

To begin with, the matrix containing peaks of the gouty patient will be stored in another matrix called U. Thereafter, the code then runs through the matrix that contain the peaks for the gouty patient, row by row, in order to check if a value from MSU standard within the permissible shift is present in $U$ and will then be printed on the display.

\subsubsection{Syntax for peak comparison}

In the code below, the matrix with peaks for the gouty patient were stored in a matrix called U. Then a matrix of zeroes was created, which had a size of (rows in U) x (18), as there were a total of 18 peaks in the MSU standard. Thereafter, two matrixes were created named: upperlimit and lowerlimit that had a size of $18 \times 1$. Furthermore, the upperlimit and lowerlimit matrixes were similar to peaklist_S, where the difference being that the upperlimit matrix was equal to an addition of four to each row of peaklist_S matrix, while in constrast the lowerlimit matrix was equal to a subtraction of four from each row of peaklist_S matrix.

U=peaklist_G(:,1);

ind $=1$;

$\mathbf{s z}=\operatorname{size}(\mathbf{U})$;

$\mathrm{L}=\operatorname{zeros}(\mathbf{s z}(:, 1), 18)$;

lowerlimit=peaklist_S(:,1)-4;

upperlimit=peaklist_S(:,1)+4; 
The following for loop checks whether column one in the gouty peaklist matrix has wavenumbers that are present in row number $=$ ind for the upperlimit and lowerlimit matrixes that have a value within the range in question. For example, when the loop begins, the value of ind was one, therefore the range was between 382 and 390, where 382 was the value in row one of the lowerlimit matrix, while on the other hand, 390 was the value in row one of the upperlimit matrix. Additionally, if there was a value within this range in the matrix $U$, then the column number will equal 'ind', in matrix L had a value of one, appearing once. Whereas, if no value was found then the result in every row of column number that equals 'ind' will be a value of zero.

for ind $=1: 18$

$\mathrm{L}($ :,ind $)=((\mathrm{U}(:, 1)>=$ lowerlimit(ind,:))\& $(\mathrm{U}(:, 1)<=$ upperlimit(ind,:)));

end

The following for loop checks every column of matrix L, to see if it has a value of 1 , as this represents the presence of a peak. Furthermore, a column in matrix L corresponds directly to a row in matrix peaklist_S (MSU standard). As a result, L matrix has 18 columns and peaklist_S has 18 rows. For example if a value of one is detected in column one of matrix L, then this would mean that a peak in row one of matrix peaklist_S was present in the gouty patient. the loop will check all columns in matrix L and then display the peaks that were present in the RS scan. Furthermore, the loop will also determine the location and intensity of the peak detected and store in variables Peakpresent and Peakintensity, respectively.

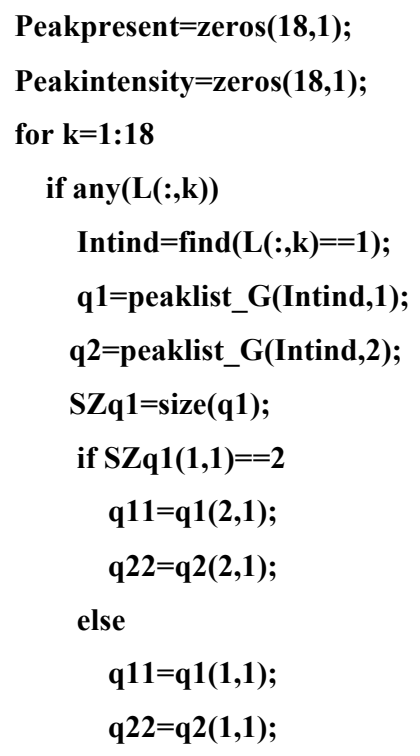




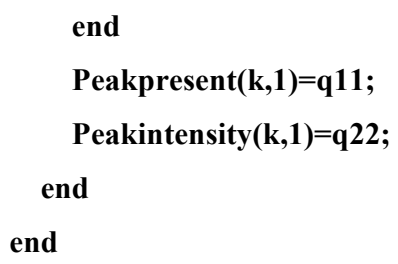

\subsubsection{Data export}

The final step of the algorithm will export the data using built-in Matlab functions. The function 'table' will be used to organize the required outputs in a form of a table, after which 'writetable' will be utilized to create a spreadsheet with the assigned named. Each sheet will correspond to a new scan and each sheet will mention the original file name that corresponds to the patient number. The following code will be used to execute the data export:

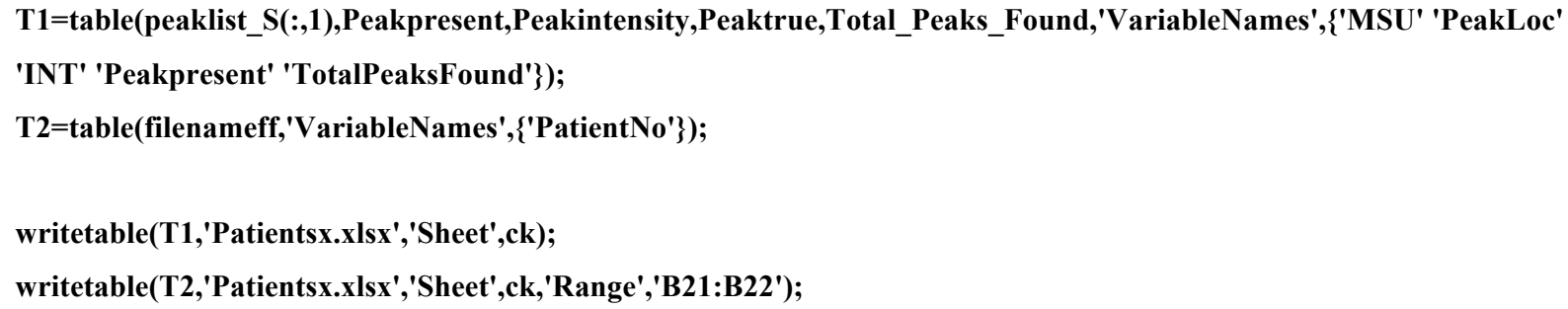

\subsection{Peak combinations}

All possible peak combinations of upto 6 peaks were used to conduct a brute force search that gives the highest sensitivity and specificity value for both studies. The priority was given to the sensitivity over the specificity. The formulas for determining the sensitivity and specificity are given below.

\section{Sensitivity:}

Is the true positive rate, which was determined by:

$$
\frac{\text { True positive }}{\text { True positive }+ \text { False negative }}
$$

\section{Specificity:}

Is the true negative rate, which was determined by:

$$
\frac{\text { True negative }}{\text { True negative }+ \text { False positive }}
$$




\section{Chapter 6: Results}




\subsection{Demographics of the pilot study (Pilot)}

A total of 20 subjects participated in the study, of which 10 had been previously clinically diagnosed with gout, and undergoing treatment, while 10 had not, but were diagnosed with, and under treatment for, Osteoarthritis (OA). The mean age of all subjects was 72.75 years with a mean Serum urate of $267 \mu \mathrm{mol} / \mathrm{l}$. A total of 85 Raman spectroscopy (RS) scans were collected, with a mean of 4.25 scans per patient. Further details on age, gender, treatments and Serum urate levels are outlined in Table 11.

Table 11. Pilot study characteristics $(n=20)$.

\begin{tabular}{|c|c|c|}
\hline Characteristic & gout & $\boldsymbol{O A}$ \\
\hline Subjects, $n$ & 10 & 10 \\
\hline Age, mean (S.D), years & $68.7(10)$ & $76.8(4.4)$ \\
\hline Sex, male, $n(\%)$ & $8(80)$ & $3(30)$ \\
\hline Serum urate, mean (S.D), $\mu \mathrm{mol} / \mathrm{l}$ & $221(31.8)$ & $313(87.9)$ \\
\hline Serum urate $>360, \mu \mathrm{mol} / \mathrm{l}, \mathrm{n}$ & 0 & 2 \\
\hline Duration of gout, median (IQR), years & $8.5(8)$ & - \\
\hline Time since started on ULT, median (IQR), months & $12(6)$ & - \\
\hline Current allopurinol dose, median $(I Q R)^{a}$ & $500(0)$ & - \\
\hline Time since last acute gout, median (IQR), months & $6(5)$ & - \\
\hline Time since normal serum urate, median (IQR), months & $10(6)$ & - \\
\hline Meets clinical classification criteria for gout, ${ }^{b} n$ & 10 & 0 \\
\hline Total RS scans collected & 44 & 41 \\
\hline RS Scans per patient, mean, $n$ & 4.4 & 4.1 \\
\hline
\end{tabular}

$-{ }^{a}$ three patients were on febuxostat. ${ }^{b}$ As per Pelaez- Ballestas et al [81].; ULT: urate- lowering treatment. 


\subsubsection{Gout Subjects SUA levels for pilot study}

SUA levels were negatively associated with average Raman peaks detected per scan, but the relationship was not significant $(\mathrm{R}=-0.601, \mathrm{p} \leq 0.066)$. On Average 12.8(1.32) peaks were detected per subject. The list of subjects with their corresponding SUA levels and average Raman peaks detected per scan, are shown in Table 12.

Table 12. SUA levels and Average Raman peaks detected per scan for gout subjects.

\begin{tabular}{|l|l|l|}
\hline Subject \# & SUA level $\mu$ mol/l & Average Raman peaks detected \\
& & per scan mean (Std) \\
\hline $\mathbf{1}$ & 249 & $13.3(0.6)$ \\
\hline $\mathbf{3}$ & 223 & $11.4(1.8)$ \\
\hline $\mathbf{6}$ & 179 & $14(1.8)$ \\
\hline $\mathbf{7}$ & 227 & $14.3(4.3)$ \\
\hline $\mathbf{1 1}$ & 290 & $10.4(2.6)$ \\
\hline $\mathbf{1 2}$ & 212 & $13.8(0.5)$ \\
\hline $\mathbf{1 6}$ & 219 & $13.3(1.6)$ \\
\hline $\mathbf{1 7}$ & 226 & $11.3(2.6)$ \\
\hline $\mathbf{1 8}$ & 198 & $12.5(1.3)$ \\
\hline $\mathbf{1 9}$ & 188 & \\
\hline
\end{tabular}

6.1.2 OA subjects SUA levels for pilot study

SUA levels for OA subjects were negatively associated with average Raman peaks detected per scan, but the relationship was not significant $(R=-0.595, \mathrm{p} \leq 0.069)$. On average 10.9(1.10) peaks were detected per subject. The list of subjects with their corresponding SUA levels and average Raman peaks detected per scan, are shown in Table 13. 
Table 13. SUA levels and Average Raman peaks detected per scan for OA subjects.

\begin{tabular}{|l|l|l|}
\hline Subject \# & SUA level $\mu$ mol/l & Average Raman peaks detected per scan mean(Std) \\
\hline $\mathbf{1}$ & 313 & $12(1.8)$ \\
\hline $\mathbf{4}$ & 309 & $11(2.4)$ \\
\hline $\mathbf{5}$ & 150 & $11.8(2.1)$ \\
\hline $\mathbf{8}$ & 432 & $10(1.8)$ \\
\hline $\mathbf{9}$ & 349 & $10.5(1.9)$ \\
\hline $\mathbf{1 0}$ & 321 & $9.8(1.0)$ \\
\hline $\mathbf{1 3}$ & 305 & $9.4(2.9)$ \\
\hline $\mathbf{1 4}$ & 210 & $13(1.0)$ \\
\hline $\mathbf{1 5}$ & 442 & $10.6(1.3)$ \\
\hline $\mathbf{2 0}$ & 306 & \\
\hline
\end{tabular}

\subsubsection{Peak statistics for pilot study}

The frequency for all expected MSU peaks, were recorded and their corresponding percentage of occurrence was calculated for gout and OA subjects, which were determined from [43] and are shown in Figure 42. Total possible occurrences for gouty and OA subjects were 44 and 41, respectively. In addition, the differences in occurrence percentages were also calculated and are shown in Table 14. There are 13 peaks that show a significant difference between both populations, when considering the significant difference to be those that are greater than 5\%. 12 of these have a higher occurrence $\%$ for gouty subjects, where as one of these has a higher occurrence $\%$ for OA subjects. Overall, 15(83\%) of the MSU peaks show a higher occurrence \% for gout subjects over OA subjects. 
Table 14. Peak occurrences for pilot study $(n=20)$.

\begin{tabular}{|c|c|c|c|c|c|}
\hline \multirow[t]{2}{*}{$\operatorname{Peak}\left(\mathrm{cm}^{-1}\right)$} & \multicolumn{2}{|c|}{ gout subjects $(n=10)$} & \multicolumn{2}{|c|}{$O A$ subjects $(n=10)$} & \multirow[b]{2}{*}{$\begin{array}{l}\text { Difference } \\
(\%)\end{array}$} \\
\hline & $\begin{array}{l}\text { Number of } \\
\text { occurrences (n) }\end{array}$ & Occurrence (\%) & $\begin{array}{l}\text { Number of } \\
\text { occurrences (n) }\end{array}$ & $\begin{array}{l}\text { Occurrence } \\
(\%)\end{array}$ & \\
\hline 386 & 21 & 47.7 & 13 & 31.7 & 16 \\
\hline 403 & 30 & 68.2 & 24 & 58.5 & 9.7 \\
\hline 492 & 35 & 79.5 & 22 & 53.7 & 25.8 \\
\hline 591 & 32 & 72.7 & 28 & 68.3 & 4.4 \\
\hline 632 & 28 & 63.6 & 26 & 63.4 & 0.2 \\
\hline 689 & 33 & 75.0 & 25 & 61.0 & 14 \\
\hline 749 & 34 & 77.3 & 33 & 80.5 & -3.2 \\
\hline 788 & 28 & 63.6 & 14 & 34.1 & 29.5 \\
\hline 878 & 32 & 72.7 & 27 & 65.9 & 6.8 \\
\hline 1014 & 27 & 61.4 & 22 & 53.7 & 7.7 \\
\hline 1063 & 31 & 70.5 & 27 & 65.9 & 4.6 \\
\hline 1130 & 34 & 77.3 & 26 & 63.4 & 13.9 \\
\hline 1209 & 35 & 79.5 & 35 & 85.4 & -5.9 \\
\hline 1369 & 33 & 75.0 & 21 & 51.2 & 23.8 \\
\hline 1419 & 27 & 61.4 & 26 & 63.4 & -2 \\
\hline 1450 & 36 & 81.8 & 27 & 65.9 & 15.9 \\
\hline 1502 & 28 & 63.6 & 19 & 46.3 & 17.3 \\
\hline 1600 & 35 & 79.5 & 27 & 65.9 & 13.6 \\
\hline
\end{tabular}

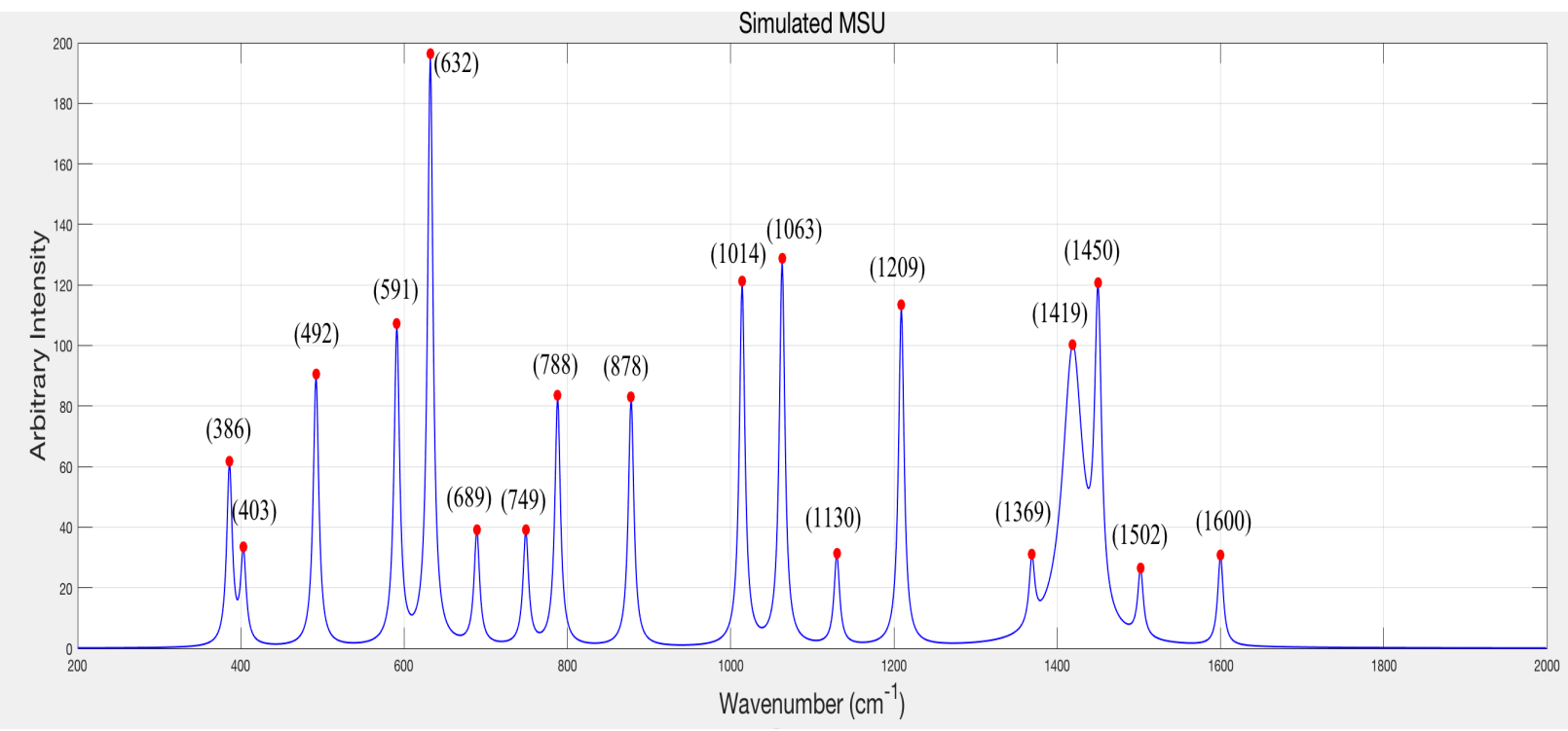

Figure 42. Simulated MSU with labelled Raman peak wavenumbers, recreated from [43]. 


\subsubsection{Raman scan examples for Pilot}

In this following section, examples of Raman scans for gouty subject and Non-gouty (OA) subject are shown for the pilot study. In Figure 43, a Raman scan for a gouty subject is shown overlaid with a MSU standard spectrum and similarly in Figure 44, a Raman scan for Non-gouty subject is shown. A gouty against Non-gouty (OA) Raman scan is shown in Figure 45.

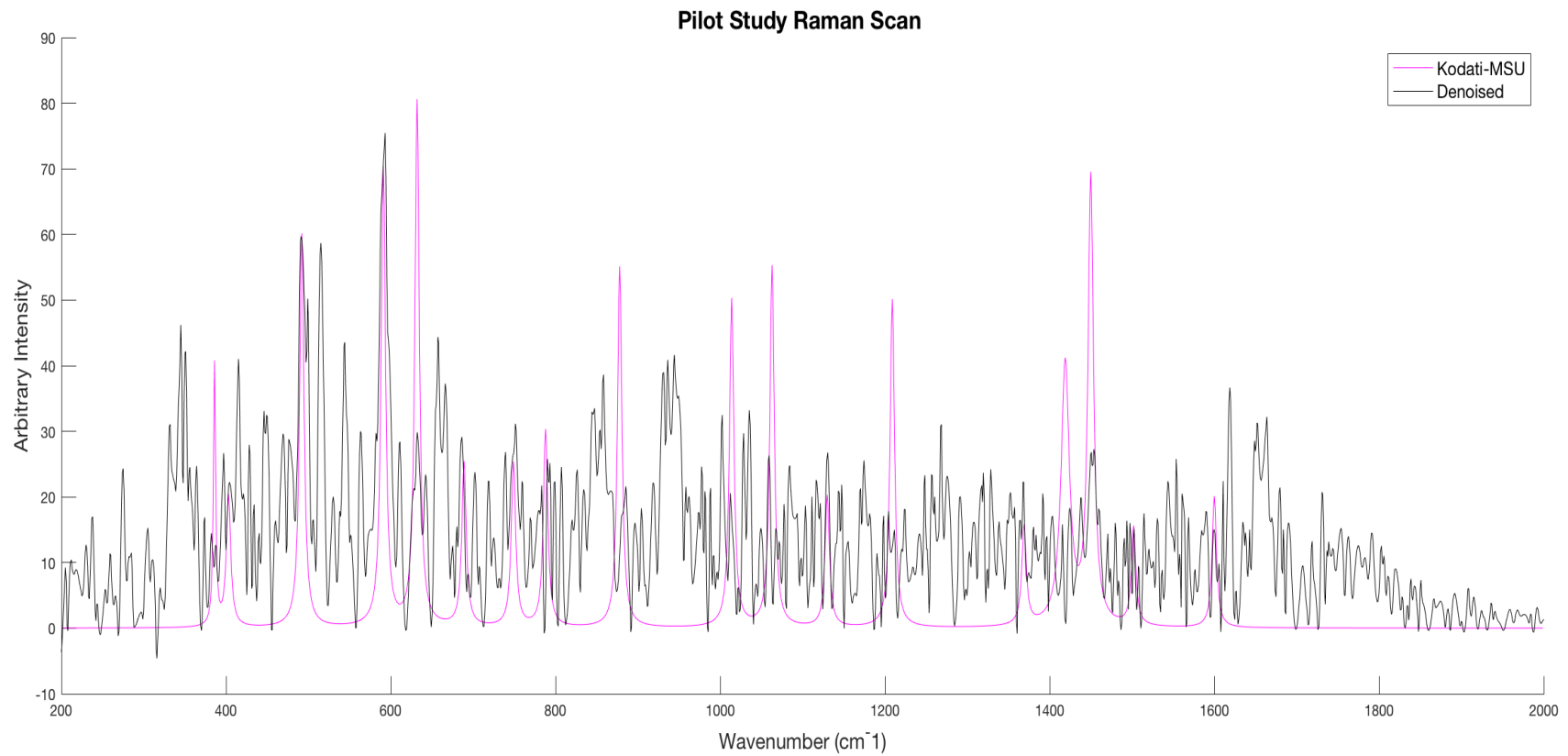

Figure 43. gouty subject example of de-noised Raman scan with MSU trace overlaid.

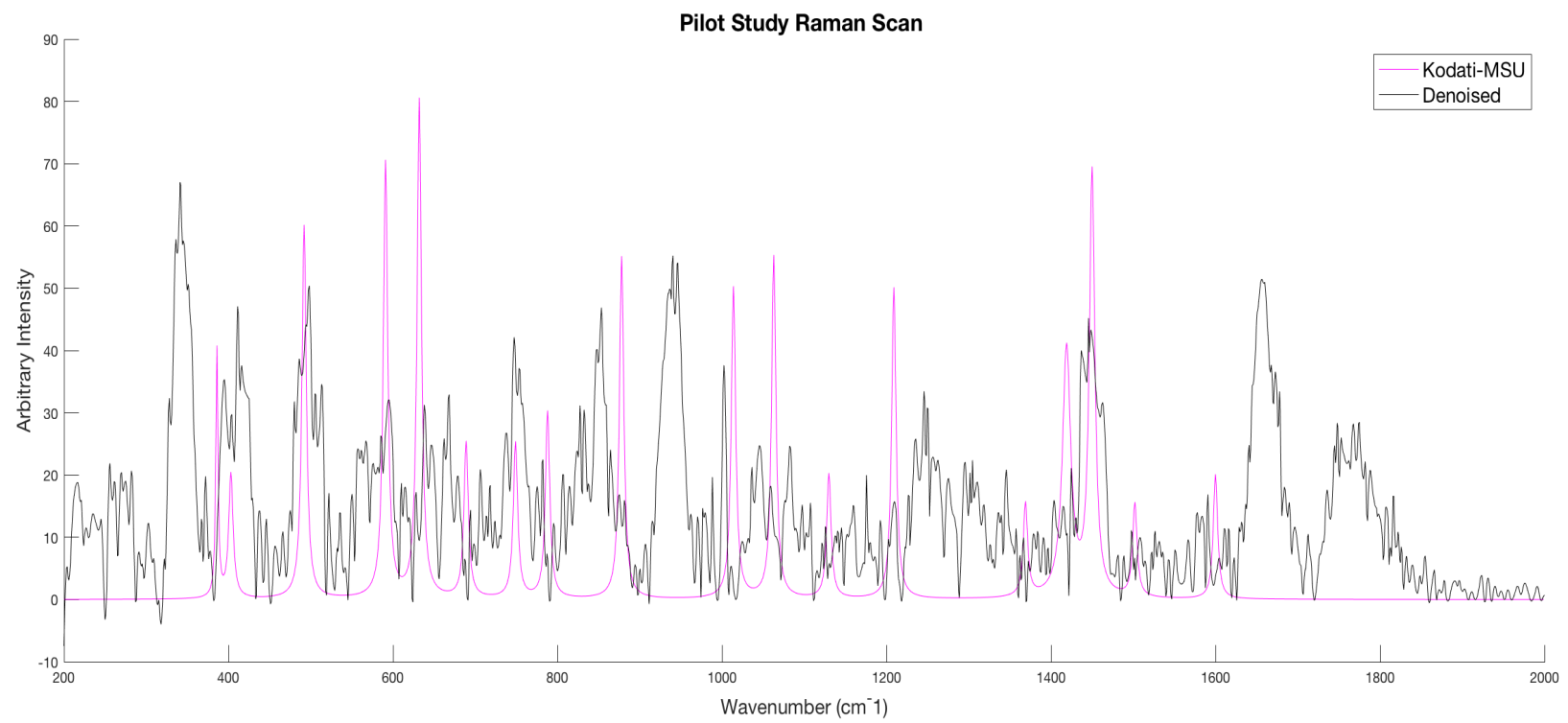

Figure 44. Non-gouty (OA) subject example of de-noised Raman scan with MSU trace overlaid. 


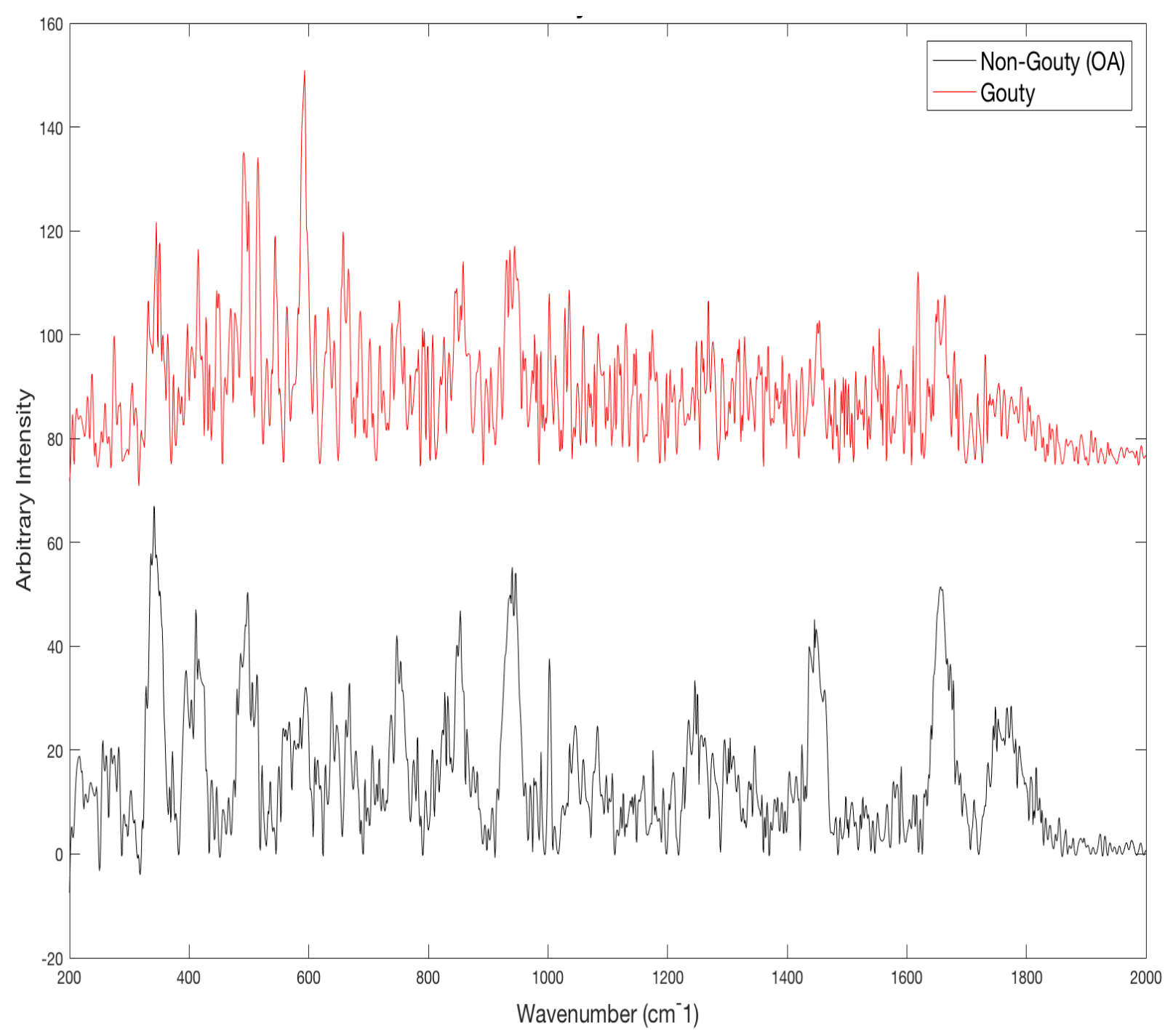

Figure 45. gouty vs Non-gouty (OA) subject example with their respective de-noised Raman scans.

\subsection{Demographics of the SOG study}

A total of 25 subjects participated in the study, of which 9 showed signs of gout under ultrasound, such as DCS or tophi signal, while 16 did not, which resulted in a disease prevalence of $36 \%$. None of the gouty subjects were diagnosed gout sufferers, but rather the sons of patients treated for gout, essentially comparing US results to RS results. The mean Serum urate was $371.8 \mu \mathrm{mol} / \mathrm{l}$. A total of $144 \mathrm{RS}$ scans were collected, with a mean of 5.76 scans per patient. Further details on Serum urate levels, scans collected, ultrasound features and subjects are outlined in Table 15 and the SUA level distribution for the study is given in Figure 46. 
Table 15. SOG study characteristics $(n=25)$.

\begin{tabular}{|c|c|c|}
\hline Characteristic & gout & Non-gouty \\
\hline Subjects, $\boldsymbol{n}$ & 9 & 16 \\
\hline Serum urate, mean (S.D), $\boldsymbol{\mu m o l / \boldsymbol { l }}$ & $376.8(46.5)$ & $369(70.8)$ \\
\hline Serum urate $>\mathbf{3 6 0}, \boldsymbol{\mu m o l / / ,}, \boldsymbol{n}(\%)$ & $6(66.7)$ & $9(56.3)$ \\
\hline DCS observed, $\boldsymbol{n}(\%)$ & $7(77.8)$ & $0(0)$ \\
\hline Tophus observed, $\boldsymbol{n}(\%)$ & $4(44.4)$ & $0(0)$ \\
\hline HEDs observed, $\boldsymbol{n}(\%)$ & $6(66.7)$ & $8(50)$ \\
\hline Effusion observed, $\boldsymbol{n}(\%)$ & $7(77.8)$ & $6(37.5)$ \\
\hline Meets classification criteria $\boldsymbol{a}, \boldsymbol{n}$ & 9 & 16 \\
\hline Total RS scans collected & 53 & 91 \\
\hline RS Scans per patient, mean, $\boldsymbol{n}$ & 5.89 & 5.69 \\
\hline
\end{tabular}

${ }^{a}$ Classification criteria stated in 5.2.2 SOG Study.; ULT: urate- lowering treatment.

\section{Fiber study- Population SUA levels}

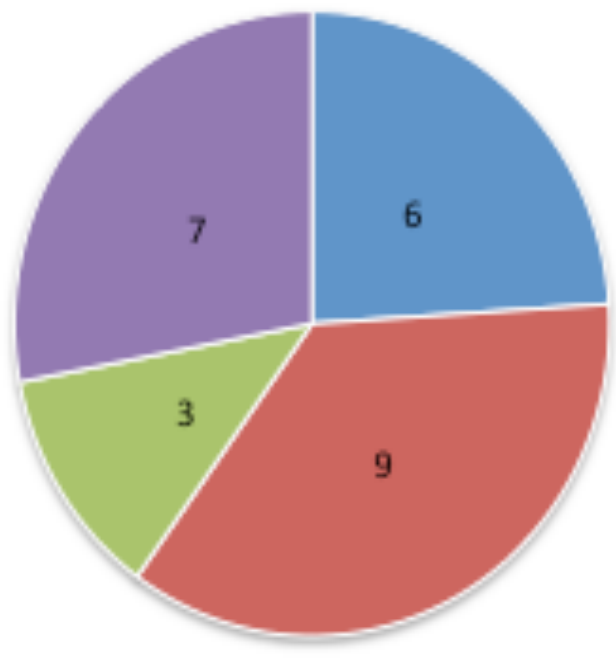

" Gouty $>360 \mu \mathrm{mol} / \mathrm{l}$

" Non-Gouty $>360 \mu \mathrm{mol} / \mathrm{l}$

" Gouty $<360 \mu \mathrm{mol} / \mathrm{l}$

" Non-Gouty $<360 \mu \mathrm{mol} / \mathrm{l}$

Figure 46. SUA level distribution for SOG study, gouty and Non-gouty populations.

\subsubsection{Ultrasound features}

For all 25 subjects, ultrasound was used as a validation technique. The presence of features such as HEDs, Effusions, Double contour signals (DCS) and Tophus were recorded. The following table shows the results for all subjects. 
Table 16. Ultrasound Features observed for SOG study ( $n=25)$ for both left (L) and right (R) MTP joints.

\begin{tabular}{|c|c|c|c|c|c|c|c|c|}
\hline$I D$ & $\operatorname{DCS}(R)$ & $\operatorname{DCS}(L)$ & Tophus (R) & Tophus (L) & HEDs (R) & HEDs $(L)$ & Effusion (L) & Effusion (R) \\
\hline 1 & \multicolumn{4}{|c|}{$\mathrm{N}$} & $\mathrm{T}$ & $\mathrm{T}$ & $\mathrm{T}$ & $\mathrm{T}$ \\
\hline 2 & \multicolumn{4}{|c|}{$\mathrm{N}$} & $\mathrm{N}$ & $\mathrm{T}$ & \multicolumn{2}{|c|}{$\mathrm{N}$} \\
\hline 3 & $\mathrm{~T}$ & $\mathrm{~T}$ & $\mathrm{~N}$ & $\mathrm{~T}$ & $\mathrm{~N}$ & $\mathrm{~T}$ & $\mathrm{~T}$ & $\mathrm{~T}$ \\
\hline 4 & \multicolumn{4}{|c|}{$\mathrm{N}$} & $\mathrm{T}$ & $\mathrm{T}$ & \multicolumn{2}{|c|}{$\mathrm{N}$} \\
\hline 5 & \multicolumn{4}{|c|}{$\mathrm{N}$} & $\mathrm{N}$ & $\mathrm{T}$ & \multicolumn{2}{|c|}{$\mathrm{N}$} \\
\hline 6 & \multicolumn{4}{|c|}{$\mathrm{N}$} & $\mathrm{N}$ & $\mathrm{T}$ & \multicolumn{2}{|c|}{$\mathrm{N}$} \\
\hline 7 & \multicolumn{4}{|c|}{$\mathrm{N}$} & $\mathrm{T}$ & $\mathrm{N}$ & $\mathrm{T}$ & $\mathrm{T}$ \\
\hline 8 & \multicolumn{8}{|c|}{$\mathrm{N}$} \\
\hline 9 & \multicolumn{4}{|c|}{$\mathrm{N}$} & $\mathrm{T}$ & $\mathrm{N}$ & $\mathrm{T}$ & $\mathrm{T}$ \\
\hline 10 & $\mathrm{~T}$ & \multicolumn{3}{|c|}{$\mathrm{N}$} & $\mathrm{T}$ & $\mathrm{N}$ & $\mathrm{T}$ & $\mathrm{N}$ \\
\hline 11 & \multicolumn{2}{|c|}{$\mathrm{N}$} & $\mathrm{T}$ & $\mathrm{T}$ & \multicolumn{2}{|c|}{$\mathrm{N}$} & $\mathrm{T}$ & $\mathrm{N}$ \\
\hline 12 & $\mathrm{~T}$ & \multicolumn{7}{|c|}{$\mathrm{N}$} \\
\hline 13 & \multicolumn{8}{|c|}{$\mathrm{N}$} \\
\hline 14 & \multicolumn{2}{|c|}{$\mathrm{N}$} & $\mathrm{T}$ & \multicolumn{5}{|c|}{$\mathrm{N}$} \\
\hline 15 & \multicolumn{8}{|c|}{$\mathrm{N}$} \\
\hline 16 & \multicolumn{4}{|c|}{$\mathrm{N}$} & $\mathrm{T}$ & $\mathrm{N}$ & $\mathrm{T}$ & $\mathrm{N}$ \\
\hline 17 & \multicolumn{8}{|c|}{$\mathrm{N}$} \\
\hline 18 & $\mathrm{~T}$ & $\mathrm{~T}$ & \multicolumn{2}{|c|}{$\mathrm{N}$} & $\mathrm{T}$ & $\mathrm{N}$ & $\mathrm{T}$ & $\mathrm{N}$ \\
\hline 19 & $\mathrm{~T}$ & & $\mathrm{~T}$ & $\mathrm{~N}$ & $\mathrm{~T}$ & $\mathrm{~N}$ & $\mathrm{~T}$ & $\mathrm{~N}$ \\
\hline 20 & \multicolumn{6}{|c|}{$\mathrm{N}$} & $\mathrm{T}$ & $\mathrm{N}$ \\
\hline 21 & $\mathrm{~N}$ & $\mathrm{~T}$ & \multicolumn{3}{|c|}{$\mathrm{N}$} & $\mathrm{T}$ & $\mathrm{T}$ & $\mathrm{N}$ \\
\hline 22 & \multicolumn{8}{|c|}{$\mathrm{N}$} \\
\hline 23 & \multicolumn{8}{|c|}{$\mathrm{N}$} \\
\hline 24 & $\mathrm{~T}$ & $\mathrm{~N}$ & $\mathrm{~T}$ & $\mathrm{~N}$ & $\mathrm{~T}$ & $\mathrm{~N}$ & $\mathrm{~T}$ & $\mathrm{~N}$ \\
\hline 25 & \multicolumn{7}{|c|}{$\mathrm{N}$} & $\mathrm{T}$ \\
\hline
\end{tabular}

* T stands for True, indicating the feature was observed for said subject, whereas $\mathrm{N}$ means a feature was not observed. All features were observed on the MTP.

*R indicates Right MTP and L indicates Left MTP

As stated in 5.2.2 SOG Study, the criteria for considering a patient gouty was the presence of a DCS signal and/or a tophus signal. Subjects without either of these signals, were considered non-gouty. 


\subsubsection{Gout Subjects SUA levels for SOG study}

SUA levels for gouty subjects were negatively associated with average Raman peaks detected per scan and the relationship was significant $(\mathrm{R}=-0.684, \mathrm{p} \leq 0.042)$, meaning the number of MSU peaks detected decreased with rising SUAs. An average 12.1(1.01) peaks were detected per subject. The list of subjects with their corresponding SUA levels and average Raman peaks detected per scan, are shown in Table 17.

Table 17. SUA levels and Average Raman peaks detected per scan for gout subjects from the SOG study.

\begin{tabular}{|l|l|l|}
\hline Subject \# & SUA level $\mu \mathrm{mol} / \mathrm{l}$ & $\begin{array}{l}\text { Average Raman peaks } \\
\text { detected per scan mean (Std) }\end{array}$ \\
\hline $\mathbf{3}$ & 320 & $12.0(1.9)$ \\
\hline $\mathbf{1 0}$ & 464 & $10.5(1.5)$ \\
\hline $\mathbf{1 1}$ & 406 & $11.7(1.9)$ \\
\hline $\mathbf{1 2}$ & 392 & $12.2(2.4)$ \\
\hline $\mathbf{1 4}$ & 346 & $12.2(2.0)$ \\
\hline $\mathbf{1 8}$ & 373 & $11.8(1.5)$ \\
\hline $\mathbf{1 9}$ & 377 & $13.7(1.4)$ \\
\hline $\mathbf{2 1}$ & 314 & $13.7(1.2)$ \\
\hline $\mathbf{2 4}$ & 399 & $11.5(3.1)$ \\
\hline
\end{tabular}

\subsubsection{Non-gouty Subjects SUA levels for SOG study}

SUA levels of Non-gouty subjects were not associated with average Raman peaks detected per scan $(\mathrm{R}=-0.023, \mathrm{p} \leq 0.932)$. On Average 11.9(0.72) peaks were detected per subject. The list of subjects with their corresponding SUA levels and Average Raman peaks detected per scan, are shown in Table 18. 
Table 18. SUA levels and Average Raman peaks detected per scan for Non-gouty subjects from the SOG study.

\begin{tabular}{|l|l|l|}
\hline Subject \# & $\begin{array}{l}\text { SUA level } \\
\text { Hmol/l }\end{array}$ & $\begin{array}{l}\text { Average Raman peaks } \\
\text { detected per scan mean (Std) }\end{array}$ \\
\hline $\mathbf{1}$ & 246 & $11.2(0.75)$ \\
\hline $\mathbf{2}$ & 418 & $11.5(2.81)$ \\
\hline $\mathbf{4}$ & 491 & $11.7(2.66)$ \\
\hline $\mathbf{5}$ & 485 & $12.3(1.63)$ \\
\hline $\mathbf{6}$ & 412 & $11.5(1.52)$ \\
\hline $\mathbf{7}$ & 343 & $12.2(1.47)$ \\
\hline $\mathbf{8}$ & 380 & $10.5(3.54)$ \\
\hline $\mathbf{9}$ & 323 & $12.7(2.73)$ \\
\hline $\mathbf{1 3}$ & 400 & $13.3(2.58)$ \\
\hline $\mathbf{1 5}$ & 372 & $12.3(2.94)$ \\
\hline $\mathbf{1 6}$ & 412 & $12.0(1.55)$ \\
\hline $\mathbf{1 7}$ & 339 & $11.0(2.53)$ \\
\hline $\mathbf{2 0}$ & 255 & $12.5(1.38)$ \\
\hline & 394 & $12.5(1.64)$ \\
\hline & & $12.0(2.12)$ \\
\hline & & \\
\hline & & \\
\hline & & \\
\hline
\end{tabular}

\subsubsection{Peak statistics for SOG study}

The frequency for all MSU Peaks were recorded, similarly to the pilot study and their corresponding percentage of occurrence was calculated for gouty and non-gouty subjects. Total possible occurrences for gouty and non-gouty subjects were 53 and 91, respectively. In addition, the differences in occurrence percentages were also calculated and are shown in Table 19. 
Table 19. Peak occurrence for the SOG study ( $n=25)$.

\begin{tabular}{|c|c|c|c|c|c|}
\hline \multirow{2}{*}{$\begin{array}{l}\text { Peak } \\
\left(\mathrm{cm}^{-1}\right)\end{array}$} & \multicolumn{2}{|l|}{ gouty subjects } & \multicolumn{2}{|c|}{ Non-gouty subjects } & \multirow[b]{2}{*}{$\begin{array}{l}\text { Difference } \\
(\%)\end{array}$} \\
\hline & Number of occurrences ( $n$ ) & Occurrence (\%) & $\begin{array}{l}\text { Number of } \\
\text { occurrences } \\
\text { (n) }\end{array}$ & $\begin{array}{l}\text { Occurrence } \\
(\%)\end{array}$ & \\
\hline 386 & 16 & 30.2 & 34 & 37.4 & -7.2 \\
\hline 403 & 37 & 69.8 & 62 & 68.1 & 1.7 \\
\hline 492 & 35 & 66.0 & 62 & 68.1 & -2.1 \\
\hline 591 & 41 & 77.4 & 73 & 80.2 & -2.8 \\
\hline 632 & 39 & 73.6 & 58 & 63.7 & 9.9 \\
\hline 689 & 43 & 81.1 & 65 & 71.4 & 9.7 \\
\hline 749 & 35 & 66.0 & 65 & 71.4 & -5.4 \\
\hline 788 & 38 & 71.7 & 64 & 70.3 & 1.4 \\
\hline 878 & 48 & 90.6 & 72 & 79.1 & 11.5 \\
\hline 1014 & 22 & 41.5 & 43 & 47.3 & -5.8 \\
\hline 1063 & 44 & 83.0 & 70 & 76.9 & 6.1 \\
\hline 1130 & 38 & 71.7 & 59 & 64.8 & 6.9 \\
\hline 1209 & 44 & 83.0 & 69 & 75.8 & 7.2 \\
\hline 1369 & 28 & 52.8 & 55 & 60.4 & -7.6 \\
\hline 1419 & 18 & 34.0 & 25 & 27.5 & 6.5 \\
\hline 1450 & 28 & 52.8 & 61 & 67.0 & -14.2 \\
\hline 1502 & 43 & 81.1 & 73 & 80.2 & 0.9 \\
\hline 1600 & 46 & 86.8 & 79 & 86.8 & 0 \\
\hline
\end{tabular}

There are 12 peaks that show a significant difference between both populations, when considering the significant difference to be those that are greater than $5 \%$. Seven of these have a higher occurrence $\%$ for gouty subjects; whereas five of these have a higher occurrence $\%$ for non-gouty subjects. Overall, $10(56 \%)$ of the MSU peaks show a higher occurrence \% for gout subjects over Non-gouty subjects. 


\subsubsection{Raman scan examples for SOG study}

In this following section, example of Raman scans for gouty subject and Non-gouty subject are shown for SOG. In Figure 47, a Raman scan for a gouty subject is shown overlaid with a MSU standard spectrum and similarly in Figure 48, a Raman scan for Non-gouty subject is shown. A gouty against Non-gouty Raman scan is shown in Figure 49.

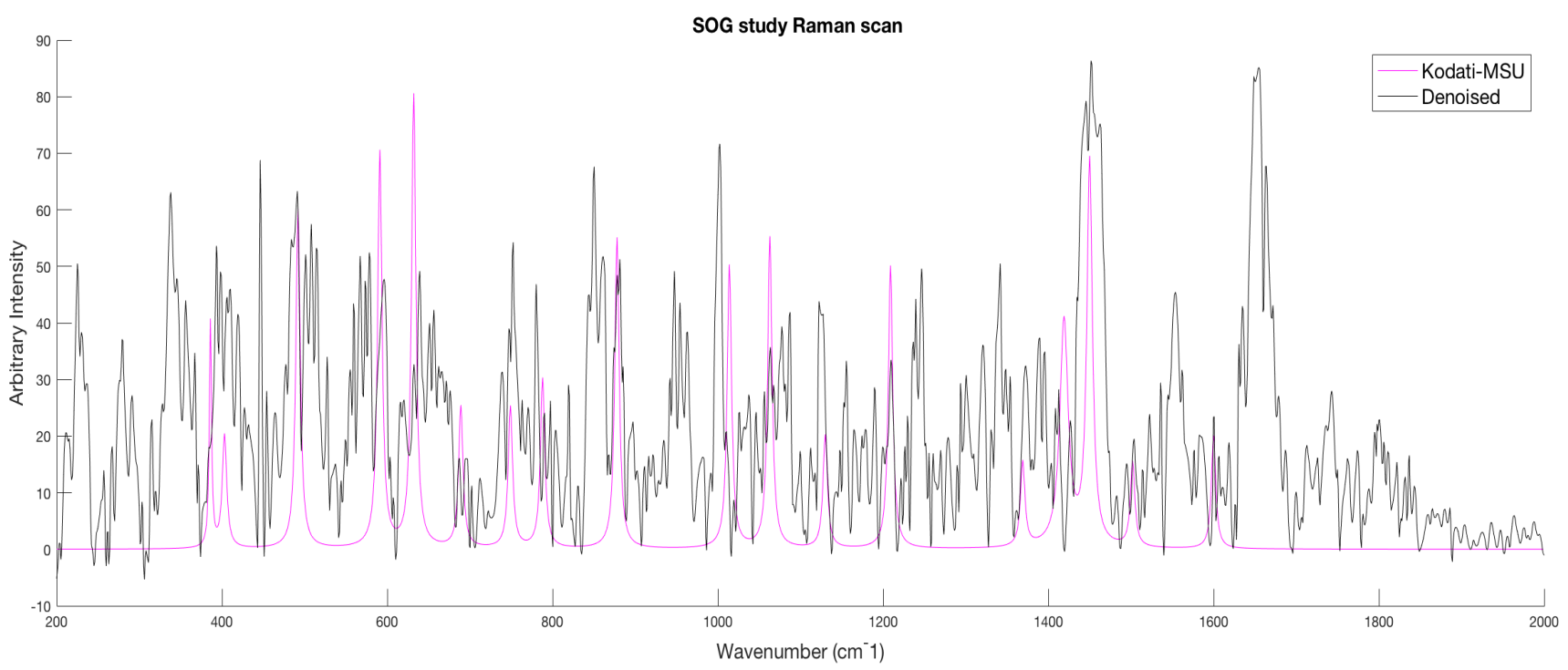

Figure 47. Gouty subject example of a denoised Raman scan with MSU trace overlaid.

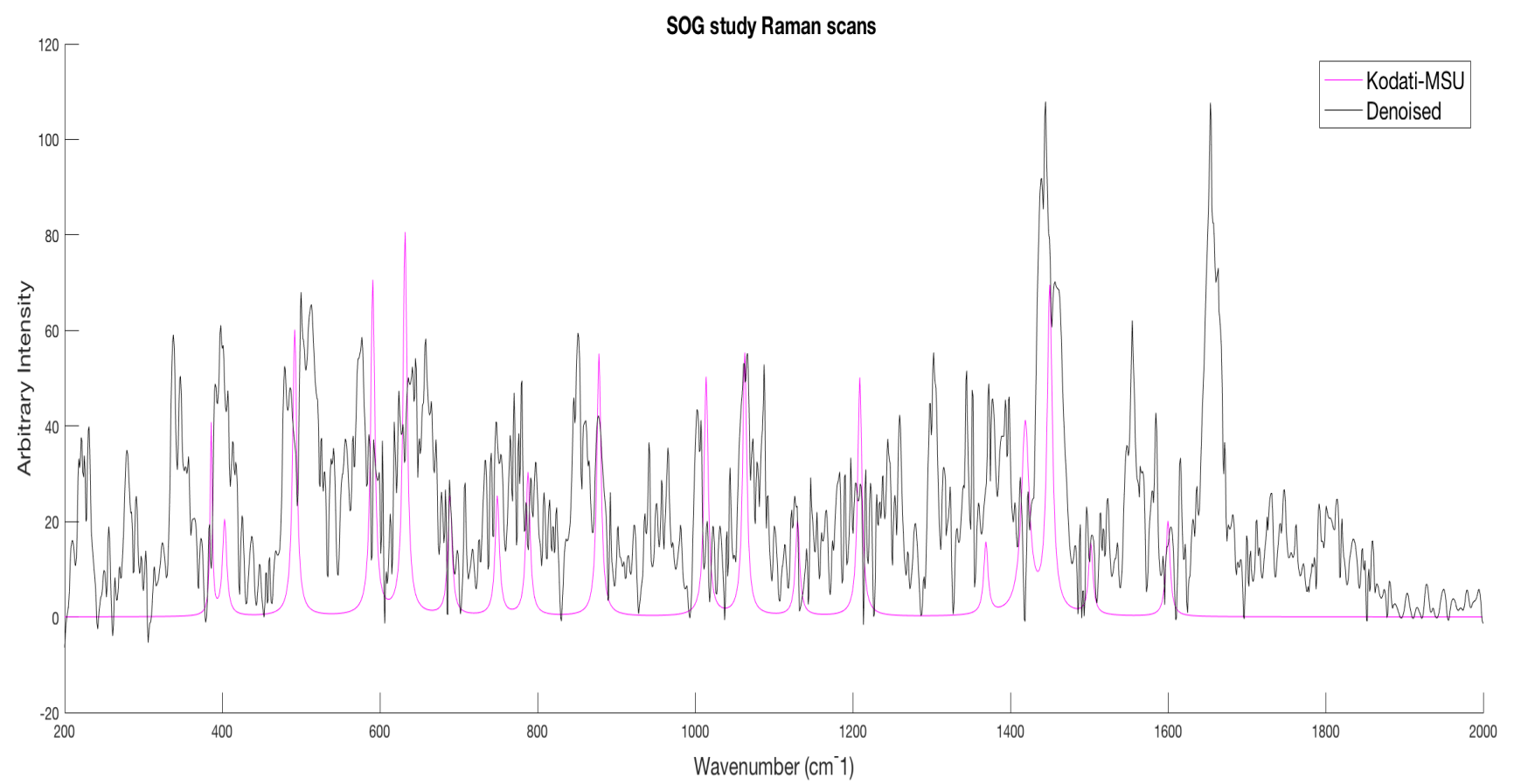

Figure 48. Non-gouty subject example of denoised Raman scan with MSU trace overlaid. 


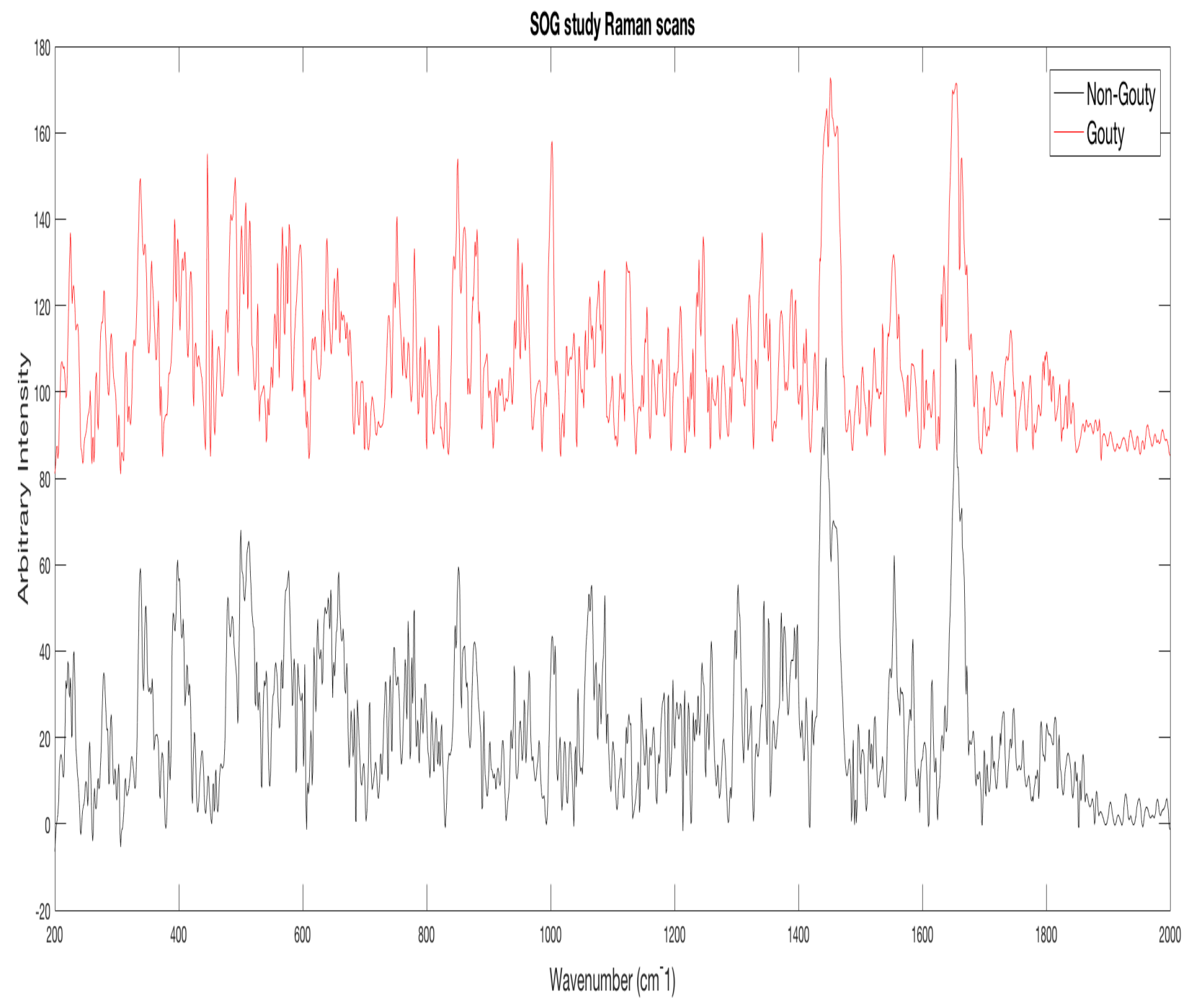

Figure 49. Non-gouty vs gouty example subject with their respective denoised Raman scans.

\subsection{Peak combinations}

Multiple combinations were tested using the combinator.m program in Appendix B: Matlab code, to determine which set of peaks are indicative of gout. The program determines the sensitivity and specificity obtained from each combination. Combinations that were below a sensitivity of 1 were removed; further narrowing of combinations was achieved based on a more favourable specificity. Combinations were determined based on the SOG study result only. The combinations matching the conditions mentioned above are shown in Table 20. 
Table 20. Peak combinations for Raman positive diagnosis.

\begin{tabular}{|c|c|c|c|c|c|c|}
\hline Combination ID & \multicolumn{7}{|c|}{ Peak wavenumbers $_{\left(\mathbf{c m}^{-1}\right)}$} \\
\hline C1 & 492 & 632 & 878 & 1130 & 1209 & 1450 \\
\hline C2 & 492 & 632 & 878 & 1130 & 1209 & 1502 \\
\hline C3 & 492 & 632 & 1130 & 1209 & 1502 & 1600 \\
\hline
\end{tabular}

The sensitivity, specificity and p-value (Fishers exact test) were determined for each of the combinations in Table 20, the results of which are shown in Table 21.

Table 21. Combination results for both studies.

\begin{tabular}{|c|c|c|c|c|c|c|}
\hline \multirow{2}{*}{ Combination ID } & \multicolumn{3}{|c|}{ SOG study } & \multicolumn{3}{c|}{ pilot study } \\
\cline { 2 - 7 } & Sensitivity & Specificity & P-value & Sensitivity & Specificity & $P$-value \\
\hline C1 & 1.0 & 0.50 & $\mathrm{P} \leq 0.008$ & 0.8 & 0.7 & $\mathrm{P} \leq 0.069$ \\
\hline C2 & 1.0 & 0.50 & $\mathrm{P} \leq 0.008$ & 0.7 & 0.8 & $\mathrm{P} \leq 0.069$ \\
\hline C3 & 1.0 & 0.50 & $\mathrm{P} \leq 0.008$ & 0.7 & 0.7 & $\mathrm{P} \leq 0.179$ \\
\hline
\end{tabular}

$*$ p-values $<0.05$ were considered significant

All three combinations for the SOG study were seen in $9(100 \%)$ of the gouty subjects and in $7(44 \%)$ of the Non-gouty subjects $(\mathrm{P} \leq 0.008)$. A sensitivity of 1 with all combinations for the SOG study shows that RS correctly identified all US positive conditions. Whereas the specificity for the SOG study was moderate to weak in its ability to correctly identify negative conditions.

The pilot study performed moderately well for $\mathrm{C} 1$ and $\mathrm{C} 2$, where $8(80 \%)$ and $7(70 \%)$ gouty subjects, respectively, were identified correctly $(\mathrm{P} \leq 0.069)$. However, C3 performed the weakest with $7(70 \%)$ of the gouty subjects identified correctly, but with a lower specificity $(\mathrm{P} \leq 0.179)$. The sensitivity and specificity values obtained were moderate to strong.

\subsection{SOG study Combination outcomes.}

\subsubsection{C1 outcome}

The diagnostic outcomes for all three combinations were similar and are shown in Table 22. Ratios associated with evaluating diagnostic outcomes are shown in Table 23. 
Table 22. Diagnostic results for SOG study combinations.

\begin{tabular}{|c|c|c|c|c|c|}
\hline $\begin{array}{l}\text { True } \\
+ \text { ves }\end{array}$ & $\begin{array}{l}\text { True - } \\
\text { ves }\end{array}$ & $\begin{array}{l}\text { False } \\
+ \text { ves }\end{array}$ & $\begin{array}{l}\text { False- } \\
\text { ves }\end{array}$ & $\begin{array}{l}\text { Total US Positive Condition } \\
\text { (gouty) }\end{array}$ & $\begin{array}{l}\text { Total US negative Condition } \\
\text { (Non-gouty) }\end{array}$ \\
\hline 9 & 9 & 7 & 0 & 9 & 16 \\
\hline
\end{tabular}

The combinations were successfully able to identify $100 \%$ of the US identified positive conditions, as evidenced by the true +ves, false -ves and the sensitivity mentioned earlier. However, the false +ves occurring for $44 \%$ of the Non-gouty population is the reason for a low specificity of 0.5 , meaning that about $50 \%$ of the Non-gouty population was correctly identified by RS as true -ves.

Table 23. diagnostic statistics for SOG study combinations.

\begin{tabular}{|l|l|l|l|l|l|l|}
\hline $\begin{array}{l}\text { Overall } \\
\text { accuracy }\end{array}$ & $\begin{array}{l}\text { Positive } \\
\text { predictive } \\
\text { value (PPV) }\end{array}$ & $\begin{array}{l}\text { Negative } \\
\text { predictive } \\
\text { value (NPV) }\end{array}$ & $\begin{array}{l}\text { FDR(False } \\
\text { discovery } \\
\text { rate) }\end{array}$ & $\begin{array}{l}\text { FOR(False } \\
\text { Omission } \\
\text { rate) }\end{array}$ & $\begin{array}{l}\text { False } \\
\text { negative rate }\end{array}$ & $\begin{array}{l}\text { False positive } \\
\text { rate }\end{array}$ \\
\hline 0.72 & 0.563 & 1 & 0.4375 & 0 & 0 & 0.4375 \\
\hline
\end{tabular}

As shown above, the overall accuracy of the device was determined to be moderate to good. The PPV shows that just less than half of the positives detected by RS were false +ves. These false +ves are those that did not have DCS and/or Tophii present on their ultrasound results, but were positive under RS. NPV shows that all negatives detected by RS were true -ves with no false -ves. Therefore, all subjects that showed signs under ultrasound of having gout were positive under RS. FOR signifies that no false -ves were observed by RS, thereby none of the positive conditions were omitted.

\subsection{Pilot study Combinations outcomes}

\subsubsection{C1 outcomes for pilot study}

C1 was successfully able to identify $80 \%$ of the positive conditions, as evidenced by the true positives, false negatives and the sensitivity mentioned earlier. However, the false positive occurring for $30 \%$ of the OA population is the reason for a moderate specificity of 0.7 , meaning that about $70 \%$ of the OA population were correctly identified by RS, as true -ves. The diagnostic results of $\mathrm{C} 1$ are shown in Table 24. 
Table 24. Diagnostic results for pilot study for combination C1.

\begin{tabular}{|l|l|l|l|l|l|}
\hline $\begin{array}{l}\text { True } \\
+v e s\end{array}$ & $\begin{array}{l}\text { True } \\
-v e s\end{array}$ & $\begin{array}{l}\text { False } \\
+v e s\end{array}$ & $\begin{array}{l}\text { False } \\
\text { I-ves }\end{array}$ & Total Positive Condition & Total negative Condition (Control) \\
\hline 8 & 7 & 3 & 2 & 10 & 10 \\
\hline
\end{tabular}

As shown in Table 25 the overall accuracy of the device was determined to be moderate to good. The PPV of 0.73 shows that just less than one-third of the positives detected by RS were false +ves. NPV of 0.78 shows that RS did not observe negative conditions correctly, due to the presence of false -ves from Table 24. FOR of 0.22 signifies that almost $20 \%$ of the gouty population were not detected, resulting in omission of positive conditions by RS.

Table 25. Diagnostic statistics for pilot study for combination C1.

\begin{tabular}{|l|l|l|l|l|l|l|}
\hline Overall accuracy & PPV & NPV & FDR & FOR & False negative rate & False positive rate \\
\hline 0.75 & 0.73 & 0.78 & 0.27 & 0.22 & 0.20 & 0.30 \\
\hline
\end{tabular}

\subsubsection{C2 outcomes for pilot study}

C2 was successfully able to identify $70 \%$ of the positive conditions, as evidenced by the true positives, false negatives and the sensitivity mentioned earlier. However, the false +ves occurring for $20 \%$ of the OA population is the reason for the specificity of 0.8 , meaning that about $80 \%$ of the OA population was correctly identified by RS as true -ves. The diagnostic results of $\mathrm{C} 2$ are shown in Table 26

Table 26. Diagnostic results for pilot study for combination C2.

\begin{tabular}{|l|l|l|l|l|l|}
\hline $\begin{array}{l}\text { True } \\
+v e s\end{array}$ & $\begin{array}{l}\text { True } \\
-v e s\end{array}$ & $\begin{array}{l}\text { False } \\
+ \text { ves }\end{array}$ & $\begin{array}{l}\text { False }- \\
\text { ves }\end{array}$ & Total Positive Condition & Total negative Condition (Control) \\
\hline 7 & 8 & 2 & 3 & 10 & 10 \\
\hline
\end{tabular}

As shown in Table 27, the overall accuracy of the device was determined to be moderate to good. The PPV shows that just less than quarter of the positives detected by RS were false positives. NPV shows that RS did not observe all negative conditions correctly, due to the presence of False negatives from Table 26. FOR signifies that almost $30 \%$ of the gouty population were not detected, resulting in omission of positive conditions. 
Table 27. Diagnostic statistics for pilot study for combination C2.

\begin{tabular}{|l|l|l|l|l|l|l|}
\hline Overall accuracy & PPV & NPV & FDR & FOR & False negative rate & False positive rate \\
\hline 0.75 & 0.78 & 0.73 & 0.22 & 0.27 & 0.30 & 0.20 \\
\hline
\end{tabular}

\subsubsection{C3 outcomes for pilot study}

C3 was successfully able to identify $70 \%$ of the positive conditions, as evidenced by the true positives, false negatives and the sensitivity mentioned earlier. However, the false positive occurring for $30 \%$ of the $\mathrm{OA}$ population is the reason for the specificity of 0.7 , meaning that about $70 \%$ of the OA population was correctly identified by RS, as true -ves, as shown in Table 28.

Table 28. Diagnostic results for pilot study for combination C3.

\begin{tabular}{|l|l|l|l|l|l|}
\hline $\begin{array}{l}\text { True } \\
+v e s\end{array}$ & $\begin{array}{l}\text { True } \\
-v e s\end{array}$ & $\begin{array}{l}\text { False } \\
+v e s\end{array}$ & $\begin{array}{l}\text { False }- \\
\text { ves }\end{array}$ & Total Positive Conditions & Total negative Conditions (Control) \\
\hline 7 & 7 & 3 & 3 & 10 & 10 \\
\hline
\end{tabular}

As shown in Table 29, the overall accuracy was the weakest of the three combinations proposed, and was determined to be moderate to good. The PPV shows that just less than onethird of the positives detected by RS were false positives. NPV shows that RS did not observe all negative conditions correctly, due to the presence of false -ves from Table 28. FOR signifies that $30 \%$ of the gouty population were not detected, resulting in omission of positive conditions.

Table 29. Diagnostic statistics for pilot study for combination C3.

\begin{tabular}{|l|l|l|l|l|l|l|}
\hline Overall accuracy & PPV & NPV & FDR & FOR & False negative rate & False positive rate \\
\hline 0.70 & 0.70 & 0.70 & 0.30 & 0.30 & 0.30 & 0.30 \\
\hline
\end{tabular}




\section{Chapter 7: Discussion}




\subsection{Pilot study}

Pilot sensitivity and specificity ranged from 0.7-0.8. The most agreeable peak combination for the pilot study was $\mathrm{C} 1$, which had a sensitivity and specificity of 0.8 and 0.7 , respectively. C1 was the most favourable for the pilot study, due to its higher sensitivity, which in turn implies higher true positives, a lower specificity was tolerated in favour of a higher sensitivity, as false reassurance to patients can potentially be more damaging. The presence of false negatives ranged from 2-3 across all combinations. Firstly, this could have occurred due to the fact that all gout patients participating in the study were undergoing urate-lowering therapy, thereby the false negatives could merely mean that some patients do not have MSU crystal deposits on their MTP joints any longer. This is likely as OA patients (mean: $313 \mu$ mol/l) have a higher SUA level than gout patients (mean: $221 \mu \mathrm{mol} / \mathrm{l}$ ), which shows that gout patients are responding well to the Urate-lowering treatment and thereby gout patients are likely to have a reduction if not a complete removal of gout crystals. Secondly, the source for the false negative could also be the method in which the RS scans were collected, as the patient's feet had to be raised to the height of the laser outlet, while also relying on the patient to not move while the scans were being collected, since the RS device was kept stationary. This could have influenced the laser positioning, which could in turn result in RS not illuminating the MSU crystals.

Finally, the false positives ranged from 2-3 across all combinations, which could be explained due to fact that OA patients are more susceptible to developing gout [83], as joints affected by OA become predisposed to the deposition of MSU crystals and synovial effusion that occurs in OA subjects at the MTP joint can lead to an increase in urate concentration, which can then lead to MSU crystal formation [84]. Additionally, the mean SUA levels for OA patients (313 $\mu \mathrm{mol} / \mathrm{l})$ was higher than gout $(221 \mu \mathrm{mol} / \mathrm{l})$ for the pilot study, and higher SUA levels present a higher risk of developing gout [11]. Therefore, it is possible that false positives from OA subjects could indeed have developed gout as well. The false negatives $(n=2)$ of the study, were likely due to the fact that the gout patients were on ULT drugs, which reduce the SUA levels and in turn reduce crystal deposits around the joints [85]. Crystals can disappear in as little as three months after starting ULT, while in this study the mean time since starting ULT was 12 months, with the lowest duration being 6 months. Therefore, the false negatives could be because those two patients no longer have gout. 


\subsection{SOG study}

SOG sensitivity and specificity were 1 and 0.5 across all combinations. RS correctly identified all positive conditions $(n=9)$. However, the presence of false positives $(n=7)$ was the result that stands out the most. Firstly, the participants of the study were not known to have gout previously but are only sons of gout patients. Furthermore, the non-gouty subjects in the SOG study had a mean SUA level of $369 \mu \mathrm{mol} / 1$, while gouty subjects had $376.8 \mu \mathrm{mol} / 1$, which are similar and above the criteria (SUA $>360 \mu \mathrm{mol} / \mathrm{l}$ ) for clinical classification of gout from the American college of rheumatology (ACR) [81]. Additionally, 6 of the 16 Non-gouty subjects had SUA $>360 \mu \mathrm{mol} / 1$. Therefore, due to the aforementioned reasons it is entirely possible that most of the non-gouty subjects have gout but at an early stage, which ultrasound failed to detect. The absence of false negatives and a $100 \%$ detection success of true positives show that RS could be equally as accurate as Ultrasound in identifying the presence of sufficient gout to cause a DCS or tophi feature.

\subsection{Peak occurrence}

Expected MSU peaks were detected individually in all four groups of patients in both the studies, as some MSU peaks overlap with Raman bands for skin and bone, however, there was no instance where all expected MSU peaks were observed. This results in OA patients (pilot study) and Non-gouty patients (SOG study) to show some peaks indicative of MSU. This could also be a result of a positive condition being present in the controls. Raman bands for human skin occur at $700-800 \mathrm{~cm}^{-1}, 1400-1450 \mathrm{~cm}^{-1}, 850-900 \mathrm{~cm}^{-1}$ and 500-600. Human bone also has Raman bands that occur at $1450 \mathrm{~cm}^{-1}, 583 \mathrm{~cm}^{-1}, 422 \mathrm{~cm}^{-1}$ and $1100-1150 \mathrm{~cm}^{-1}$. These bands from Human skin and bone overlap with 7 expected MSU peaks. Overall, there were $12 \mathrm{MSU}$ peaks for gout subjects in the pilot study that had a frequency of occurrence of more than $5 \%$ over the OA subjects, which only had one MSU peak that had a frequency of occurrence more than $5 \%$ $\left(1209 \mathrm{~cm}^{-1}\right)$. Similarly, the SOG study had 7 MSU peaks occurred more than $5 \%$ in the gouty subjects over the non-gouty, while $5 \mathrm{MSU}$ peaks occurred more often in the non-gouty subjects. These five MSU peaks could be contributing to the high number of false positives $(n=7)$ in the SOG study. MSU, skin and bone have overlapping bands for the following ranges: $450-500 \mathrm{~cm}^{-1}$,

$700-950 \mathrm{~cm}^{-1}, 1000-1100 \mathrm{~cm}^{-1}, 1400-1450 \mathrm{~cm}^{-1}, 550-600 \mathrm{~cm}^{-1}[69]$ ] [77]. These Raman bands 
overlap with 8 of the expected MSU peaks, which could explain the occurrence of MSU peaks even in the controls for both studies.

\subsection{Comparison between clinical studies}

The aim of this research was to investigate if RS could detect Monosodium urate crystals, non-invasively, in gout sufferers. The ability of the technique is evaluated by its performance against the validation technique, either aspirate (Pilot) or ultrasound (SOG). This was achieved by observing for MSU peaks in RS traces obtained from both studies, and their resulting sensitivities and specificities. C1 was selected from the three possible combinations of $\mathrm{C} 1, \mathrm{C} 2$ and $\mathrm{C} 3$, as $\mathrm{C} 1$ gave the highest sensitivity in both studies.

The overall accuracy for the SOG study (0.72) was slightly lower than pilot study (0.75), which is the average of sensitivity and specificity. The sensitivity for the SOG study (1.0) was much higher than the pilot study (0.8), while the specificity for the SOG study (0.5) was much lower than the pilot study (0.7). The notable increase in the sensitivity in the SOG study could be due to instrument refinement, where the Raman spectroscope was fitted with a fibre-optic probe (SnRI, Wyoming) allowing for more control and flexibility while scanning. This also allowed for more extended integration times to obtain RS scans, which reduced the signal-to-noise ratio, thereby improving the quality of scans and increasing the chances of identifying MSU. Another, reason for the increase in sensitivity could be the scanning procedure, which, due to flexibility of the fibre probe, minimized the influence of patient movement on RS scans, and in turn made it possible to have three different scanning positions, increasing the probability of detecting MSU, if present. These aforementioned reasons increase the chance of detecting a gout positive subject.

The decrease in specificity in the SOG study over the pilot study could be explained by the fact that none of the participants were clinically diagnosed with gout, but rather showed signs that were from ultrasound features that relate to gout. However some studies on ultrasound's ability to detect gout has had a sensitivity as low as 0.22 [86]. One advantage of the SOG study over the pilot study is that the gouty subjects were not on any treatment, as was the case in the pilot study. SUA levels relationship with average Raman peaks detected per scan were mostly not significant except for gouty subjects from SOG, where a moderate negative relationship was observed $(\mathrm{R}=-0.684, \mathrm{p} \leq 0.042)$. This moderate relationship could be attributed to the random 
nature of Raman scattering or the variation in background fluorescence, which could result in the variation of peaks detected between patients scans.

\subsection{Limitation of the studies}

7.5.1 Pilot Study: Gout patients were on ULT in the pilot study resulting in a reduction of serum urate levels and possibly even the elimination of MSU crystals. Thereby, the gout patients cohort from Pilot could include subjects that are no longer positive for gout when the RS scans were collected. OA patients being the control group for Pilot was not ideal as these patients were positive for Osteoarthritis but were not tested by synovial fluid analysis to confirm the absence of gout, due to ethical concerns of performing aspiration on control subjects. OA patients are in a higher risk group than the average population for having gout [83]; therefore, they are not the ideal control group for such a study. Furthermore, the instrumentation was not suited for in-vivo measurements, which results in inadequate conditions when collecting data, as the subjects were required to remain with their feet raised to the laser outlet height and remain stationary while collecting scans.

7.5.2 SOG. Major drawbacks were due to the fact it was not conducted against the gold standard of diagnosing gout of detecting MSU crystals with synovial fluid analysis, due to ethical reasons, as performing an invasive technique is not viable on healthy subjects that do not show signs of gout. Furthermore, the participants were not known to have gout or any symptoms of gout, but were rather sons of gout sufferers. The root problem with the study is that it was testing two hypotheses:

1. whether RS can detect MSU at gout affected joints

2. whether the sons of patients with gout, also develop gout. Essentially, evaluating genetics as a risk factor for developing gout.

However, the second hypothesis was out of the scope of this thesis. Another drawback for the SOG study was that RS results were being compared to US, which is operator dependent and is known to have low sensitivity. In addition to drawbacks with the core aspects of the studies, there were also issues with collecting proper clinical information, such as the following:

* Pilot did not allow revisiting the patients for a follow up analysis to reassess the patients using both techniques, as SF has known to be prone to errors and operator dependence

* No information of age was provided for SOG 
* Individual ages for each subject were not provided for both studies

* Individual disease duration and time since last gout attack were not provided for the pilot study

* No information on the duration between performing the SF analysis and RS for Pilot.

* No information on any other drugs, in addition to ULT, that the subjects were prescribed.

* No recorded clinical data relating to symptoms for each individual subject for both studies. 


\subsection{Comparison of Diagnostic results against existing techniques}

The sensitivities (0.8 and 1) and specificities (0.7 and 0.5) across both studies are comparable with some existing techniques for gout detection, where in some cases the technique in this study is more favourable than some techniques and less favourable than others. In comparison with US, which tends to have $0.22-0.92$ sensitivity and $>=0.95$ specificity [86], for which RS provides a comparable sensitivity with the pilot study and a more favourable sensitivity with SOG. RS has a much-improved sensitivity than conventional X-ray (0.31). DECT has a sensitivity of $0.78-1$ and specificity of $0.79-1$, for which RS has comparable sensitivity but lower specificity. Finally, with the gold standard of SF analysis, RS has better sensitivities across both studies, where as SF analysis can have a sensitivity of 0.625-0.78 [87]. However, the specificity for SF analysis $0.93-1$ is much higher than RS.

Table 30. Comparison of RS with existing Diagnostic techniques for gout.

\begin{tabular}{|c|c|c|c|}
\hline $\begin{array}{l}\text { Diagnostic } \\
\text { Technique }\end{array}$ & Sensitivity & Specificity & Comparison with $R S$ \\
\hline $\begin{array}{l}\text { Ultrasound } \\
\text { (US) }\end{array}$ & $\begin{array}{l}0.22-0.92 \\
{[86]}\end{array}$ & $>=0.95[86]$ & $\begin{array}{l}* \text { RS does not require a trained ultrasonographer to analyze } \\
* \quad \text { Gives better sensitivity than US in the SOG study } \\
* \quad \text { Detection can be automated for RS but not US } \\
* \quad \text { RS does not require any sample preparation, where as } \\
\text { ultrasound requires ultrasound gel } \\
* \quad \text { RS provides information relating to chemical composition but } \\
\text { US does not }\end{array}$ \\
\hline X-ray & 0.31 & - & $\begin{array}{l}* \text { RS has much higher sensitivity } \\
* \quad \text { RS is portable } \\
* \quad \text { Exposes subject to radiation, which can cause cancer } \\
* \quad \text { X-ray features are not specific to gout }\end{array}$ \\
\hline DECT & $0.78-1$ & $0.79-1$ & $\begin{array}{l}\text { - DECT has limited availability and higher cost than RS } \\
\text { - DECT is not portable but RS is } \\
\text { * DECT is not suitable for early stage gout. } \\
\text { DECT has Comparable sensitivities with RS }\end{array}$ \\
\hline SF Analysis & $0.625-0.78$ & $0.93-1$ & $\begin{array}{l}\text { * SF analysis is invasive, whereas RS is not } \\
* \text { RS has better sensitivity than SF } \\
* \quad \text { RS Does not require sample preparation } \\
* \quad \text { RS analysis is not operator dependent } \\
* \quad \text { Diagnosis is immediate for RS, whereas for SF analysis takes } \\
\text { substantially more time and SF sample must be sent to the lab }\end{array}$ \\
\hline
\end{tabular}


Chapter 8: Conclusions 


\subsection{Conclusions}

* RS provides similar sensitivity to ultrasound, and as previously mentioned is comparable with techniques used for diagnosing gout, However, both studies on RS accumulated to 45 subjects in total which is minimal in comparison to studies conducted with US.

* RS had a sensitivity and specificity of 0.8 and 0.7 , respectively, for the pilot study

* RS had a sensitivity and specificity of 1 and 0.5 , respectively, for the SOG study

* Diagnosis-using RS can be automated without the need for trained operators, thereby eliminating the errors from subjectivity that usually occur with SF analysis.

* The outcome of both studies shows low specificity and high false positives; the latter $(n=7)$ observed in the SOG study was not conducted against the gold standard of MSU crystal detection in SF fluid using PLM. This was because none of the participants were known to have gout but rather sons of patients who have had gout. Therefore, performing a painful aspirate fluid extraction for SF analysis was not feasible or warranted.

* Diagnosis of gout using RS is non-invasive and can show results in real-time with further improvements in post-processing of obtained data.

* The improvement in sensitivity of SOG over Pilot is a result of the changes in equipment and test procedure.

* Larger population studies need to be conducted against the gold standard with gouty subjects that have crystal proven gout and controls that have no other comorbidities of gout, to truly gauge the potential of Raman spectroscopy as a novel non-invasive diagnostic tool for gout. 
Chapter 9: Future work and recommendations 


\subsection{Recommendations for further clinical studies:}

* Further studies should be performed with larger populations, against the gold standard of gout diagnosis and with clinically diagnosed gout subjects, as this will result in a definitive conclusion on whether RS is better alternative to existing techniques,

* Acquisition of RS scans should coincide with fluid extraction for SF analysis, minimizing the gap between diagnosis by gold standard and assessment by RS

* Data such as, age, gender, ethnicity, duration of gout and comorbidities should be well documented for all participating patients in future clinical trials, as these are possible risk factors that contribute towards the development of gout

* Studies should be performed with the purpose of discriminating MSU crystals from CPPD crystals using RS non-invasively, as the discriminative power of RS will be higher than PLM or US since RS is able to discriminate based on chemical information rather than crystal size, shape and color.

\subsection{Recommendations for Instrumentation and post processing:}

* SORS could be utilized in further studies, as its capable of eliminating the signal contribution from the surface layer of a specimen, which in this case is the human skin and contributes 7 RS bands that overlap with MSU, as well as noise.

* Post-processing should be done using multivariate analysis, such as PCA, which is extensively used in the field of study in assessing the ability of a diagnostic technique to distinguish between diseases and controls.

* PCA should be used as it is able to distinguish small details between a set of data and classify it as either diseased or control and quantify the variations between subjects or scans.

* Real-time diagnosis should be set up once a concrete peak combination is determined after further blinded studies. This will allow immediate diagnosis of gout. 


\section{Appendices}




\section{Appendix A: Published paper}

Rheumatology Advance Access published September 5, 2015 RHEUMATOLOGY

\section{Letter to the Editor (other)}

\section{doi:10.1093/rheumatology/kev339}

In vivo detection of monosodium urate crystal deposits by Raman spectroscopy-a pilot study

SIR, Gout is the most common inflammatory arthritis and results from prolonged hyperuricaemia [1]. Joint aspiration and examination of the aspirated SF is the gold standard for diagnosing gout [2]. However, joint aspiration is painful, invasive and SF examination has modest interobserver agreement for detecting MSU crystals [3]. Raman spectroscopy (RS) offers a non-invasive method for detecting MSU crystals, as each chemical possesses a unique Raman spectrum [4]. This study reports the use of RS for detecting MSU crystal deposits in the first MTP joint of gout patients. The study was approved by the University of Nottingham Medical School Ethics Committee (Nottingham, UK; reference D11092014 SoM $\mathrm{ROD}$ ) and all participants gave written informed consent as per the Declaration of Helsinki.

Ten patients with OA not known to have gout and 10 patients with gout participating in a community-based trial of nurse-led vs general practitioner-led treatment of gout participated in this study (Table 1). All patients gave blood for serum uric acid measurement and had RS. A Sierra (Snowy Range Instruments, Laramie, WY, USA) Raman spectrometer $(785 \mathrm{~nm}$, spectral resolution $4 / \mathrm{cm})$ was used. The patients were seated with their knee flexed and the index foot rested on the floor to avoid movement during testing. The RS device was positioned on the floor and angled such that the laser impacted the medial MTP joint line perpendicularly. The device was placed at a distance of $\sim 2.7 \mathrm{~cm}$ from the first MTP joint in order to position the focal point of the laser on the joint surface. The laser was directed at a point on the medial aspect of the first MTP joint that was identified by A.A., a rheumatologist with $>9$ years experience. The foot and RS device were covered to remove any light interference. The RS device was set to illuminate with a power of $80 \mathrm{~mW}$ and $10 \mathrm{~s}$ integration time, repeated five times, with a reference scan before each $10 \mathrm{~s}$ scan. The reference scan was subtracted from the recorded scan. The mean of five reference subtracted scans formed the final spectrum.

The number of peaks in the averaged Raman spectra from the first MTP joint that matched those from a Raman spectrum of MSU crystals obtained from the gouty tophus of a patient attending the Rheumatology Clinic at St Michael's Hospital, Toronto, Ontario, Canada, were counted independently by D.C. and F.B. The tophus fluid contained MSU crystals and its Raman spectrum matched previous reports [5]. The Raman spectrum of the tophus discharge was printed on an acetate sheet and overlaid on the RS traces from the patients to identify any peaks related to MSU crystals in vivo. A combination of five spectral peaks that are characteristic of MSU was chosen to increase confidence in the Raman data [5]. Five peaks relating to MSU were present in the first MTP joints of 7 of 10 gout patients and 1 of 10 patients with OA (supplementary Fig. S1, available at Rheumatology Online). The latter observation raises the possibility of asymptomatic MSU crystal deposits. As we did not look for MSU crystals in the first MTP joint by an alternative established technique, we do not know if the absence of MSU crystals in those with gout is a true or false negative finding. We believe that this is a true negative finding since patients with gout were on urate-lowering treatment and had normal serum uric acid for several months prior to RS, and the MSU crystals may have dissolved in this time (Table 1). Similarly, we do not know if the presence of peaks characteristic of MSU crystals in one control patient with $O A$ who did not meet the classification criteria for gout [6] is a false positive or a true positive finding suggesting asymptomatic MSU crystal deposition. However, as this patients' serum uric acid was $306 \mu \mathrm{mol} / \mathrm{l}$, we believe that this could be a false positive finding.

The sensitivity of RS in detecting MSU crystal deposits in the first MTP joint in this study is comparable to that of US scans [7, 8]. However, we were unable to examine the dorsal aspect of the first MTP joint because the RS device could not be positioned on this surface due to its weight $(9 \mathrm{~kg})$ and the need to keep it absolutely still during RS. Therefore it is possible that RS may have an even higher sensitivity for detecting MSU crystal deposits than US.

TABLE 1 Disease and demographic characteristics of the study participants

\begin{tabular}{|c|c|c|}
\hline Characteristic & $\begin{array}{c}\text { Gout } \\
(n=10)\end{array}$ & $\underset{(n=10)}{\text { OA }}$ \\
\hline Age, mean (S.D.), years & $68.7(10)$ & $76.8(4.4)$ \\
\hline Sex, male, $n(\%)$ & $8(80)$ & $3(30)$ \\
\hline Serum urate, mean (s.D.), $\mu \mathrm{mol} / \mathrm{l}$ & $221(31.8)$ & $313(87.9)$ \\
\hline Serum urate $>360 \mu \mathrm{mol} / \mathrm{l}, n$ & 0 & 2 \\
\hline Duration of gout, median (IQR), years & $8.5(8)$ & - \\
\hline $\begin{array}{l}\text { Time since started on ULT, } \\
\text { median (IQR), months }\end{array}$ & $12(6)$ & - \\
\hline $\begin{array}{l}\text { Current allopurinol dose, } \\
\text { median (IQR), }{ }^{a} \mathrm{mg}\end{array}$ & $500(0)$ & - \\
\hline $\begin{array}{l}\text { Time since last acute gout, } \\
\text { median (IQR), months }\end{array}$ & $6(5)$ & - \\
\hline $\begin{array}{l}\text { Time since normal serum urate, } \\
\text { median (IQR), months }\end{array}$ & $10(6)$ & - \\
\hline $\begin{array}{l}\text { Meets clinical classification } \\
\text { criteria for gout, }{ }^{\mathrm{b}} n\end{array}$ & 10 & 0 \\
\hline MSU crystal deposit on RS, $n$ & 7 & 1 \\
\hline $\begin{array}{l}\text { Bilateral MSU crystal } \\
\text { deposits on RS, } n\end{array}$ & 3 & 0 \\
\hline
\end{tabular}

Three patients were on febuxostat. ${ }^{\mathrm{b}} \mathrm{As}$ per PelaezBallestas et al. [6]. RS: Raman spectroscopy; ULT: uratelowering treatment. 
Thus further research is required to compare RS using a fibre-optic probe to allow examination of the dorsal aspect of the foot against US, joint aspiration or dualenergy CT in order to determine if it is able to reliably detect MSU crystal deposits in vivo.

\section{Rheumatology key message}

- Raman spectroscopy may offer a non-invasive method for detecting monosodium urate crystals in gout.

\section{Acknowledgements}

The authors would like to acknowledge Dr Lawrence Rubin, Consultant Rheumatologist, St Michael's Hospital, Toronto, ON, Canada, for donating the gouty tophus discharge from one of his patients and Wendy Jenkins and Sally Doherty for their help recruiting the study participants.

Funding: This research was funded by the Natural Sciences and Engineering Research Council of Canada Discovery and Idea to Innovation (I2IPJ4915-14) funds and departmental research funds at the University of Nottingham.

Disclosure statement: The authors have declared no conflicts of interest.

Abhishek Abhishek ${ }^{1}$, Declan J. Curran ${ }^{2,3}$ Faizan Bilwani ${ }^{2}$, Adrian C. Jones ${ }^{4}$, Mark R. Towler ${ }^{2,3,5}$ and Michael Doherty ${ }^{1}$

${ }^{1}$ Academic Rheumatology, University of Nottingham, Nottingham, UK, ${ }^{2}$ Department of Mechanical \& Industrial Engineering, Ryerson University, ${ }^{3} \mathrm{Li}$ Ka Shing Knowledge Institute, St Michael's Hospital, Toronto, ON, Canada, ${ }^{4}$ Department of Rheumatology, Nottingham University Hospitals NHS Trust, Nottingham, UK and ${ }^{5}$ Department of Biomedical Engineering, University Malaya, Kuala Lumpur, Malaysia
Revised version accepted 7 August 2015

Correspondence to: Abhishek Abhishek, Academic

Rheumatology, University of Nottingham, Nottingham NG5

1PB, UK. E-mail: abhishek.abhishek@nottingham.ac.uk

\section{Supplementary data}

Supplementary data are available at Rheumatology Online.

\section{References}

1 Roddy E, Doherty M. Epidemiology of gout. Arthritis Res Ther 2010;12:223.

2 McCarty DJ, Hollander JL. Identification of urate crystals in gouty synovial fluid. Ann Intern Med 1961;54: 452-60.

3 Schumacher HR Jr, Sieck MS, Rothfuss S et al. Reproducibility of synovial fluid analyses. A study among four laboratories. Arthritis Rheum 1986;29:770-4.

4 Smith $\mathrm{E}$, Dent $\mathrm{G}$. Introduction, basic theory and principles. In: Smith E, Dent G, eds. Modern Raman Spectroscopy: A Practical Approach. Chichester, UK: John Wiley \& Sons, 2004:1-20.

5 Kodati VR, Tu AT, Turumin JL. Raman spectroscopic identification of uric-acid-type kidney stone. Appl Spectrosc 1990;44:1134-6.

6 Pelaez-Ballestas I, Hernandez Cuevas C, BurgosVargas $\mathrm{R}$ et al. Diagnosis of chronic gout: evaluating the American College of Rheumatology proposal, European League Against Rheumatism recommendations, and clinical judgment. J Rheumatol 2010;37:1743-8.

7 Naredo E, Uson J, Jimenez-Palop M et al. Ultrasounddetected musculoskeletal urate crystal deposition: which joints and what findings should be assessed for diagnosing gout? Ann Rheum Dis 2014;73:1522-8.

8 Ottaviani S, Richette P, Allard A, Ora J, Bardin T. Ultrasonography in gout: a case-control study. Clin Exp Rheumatol 2012;30:499-504. 


\section{Appendix B: Matlab code}

\section{Main program:}

FileList=dir('*spc'); \% To organize all .spc file in current directory

$\mathrm{N}=$ size(FileList,1); \% To determine how many spc files are present in the current directory

$\%$ The following loop will exact the main program to the count of $\mathrm{N}$

$\%$ determined previously, essentially batch processing all spc files in the

$\%$ directory

for $\mathrm{ck}=1: \mathrm{N}$

filename=FileList(ck).name \% The name of the current spc file being processed will be stored disp(filename); \% The name of the file being processed will be displayed

$\% \%$ SolasDx-Raman analysis program

\% Written by Faizan Bilwani. Start date: 5/25/2015

$\%$ Last Edited : 2/8/2017 by Faizan Bilwani

$\%$ Program OPERATION ->

$\%$ This piece of code deals with the processing of Raman spectral data.

$\%$ Firstly, a Monosodium urate(MSU) standard spectrum will be modelled

$\%$ using a Lorentzian function. After, which the Raman spectral data of

$\%$ a gouty or non-gouty patient will be imported, storing their wavenumbers

$\%$ and intensities in their individual 1-D arrays. Then this imported

$\%$ spectrums will be background corrected using Adaptive iterative

$\%$ reweighted penalized least squares algorithm. After, which wavelet

$\%$ denoising will be used to reduce noise in the background corrected

$\%$ spectrums. These denoised spectrums will then be put through a peak

$\%$ detection function with suitable parameters, revealing major peaks.

$\%$ Lastly, the peaks found in the Gouty patient will be compared to the MSU

$\%$ standard peak locations.and the results will be exported

\% Processing Raman data with the following major steps:

\% 1- Import original scans

$\%$ 2- Remove background of scans using airPLS

$\%$ 3- Denoise scans

$\%$ 4- perform peak detection on scans

$\%$ 5- plot detected peaks for scans

$\%$ 6- Compare scan to standard MSU peaks

$\%$ 7- Determine peaks found

\% 8- Export results.

\% \% \% \% \% \% \% \% \% \% \% \% \% \% \% \% \% \% \% \% \% \% \% \% \% \% \% \%

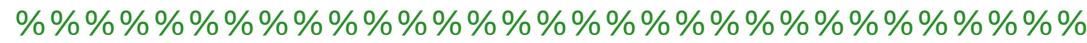

$\%$ The following three lines clear all variables and close all windows

$\%$ once the loop is executed again, except for the variables mentioned after clearvars

close all;

clearvars -except filename FileList ck N Peakwidths Peaklist_F

clc;

\% \% \% \% \% \% \% \% \% \% \% \% \% \% \% \% \% \% \% \% \% \% \% \% \% \% \%

\% \% \% \% \% \% \% \% \% \% \% \% \% \% \% \% \% \% \% \% \% \% \% \% \% \%

$\% \%$ Call \#1: Importing spectral data 
$\%$ In This section lines of code will be executed to import the required spectral

$\%$ data.

$\%$ The required input is the file name in the form of a string

$\%$ the scan stored in their appropriately

$\%$ named character arrays.

$\%$ The outputs will be arrays of the wavenumbers and intensities

Gouty= filename; \% Gouty patient

SPCStructGout = tgspcread(Gouty); \% tgspcread reads the spc files

WavenumberGout = SPCStructGout.X; \% Wavenumber of Gouty patient

IntensityGout = SPCStructGout.Y; \% Intensity of Gouty patient

$\% \% \% \% \% \%$------End of Call 1-------- $\% \% \% \% \% \%$

$\% \% \% \% \% \% \% \% \% \% \% \% \% \% \% \% \% \% \% \% \% \% \%$

$\% \%$ Call\#2: Modelling a spectrum of MSU standard

\% The MSU peak positions were taken from Kodati(1990)

$\%$ The peak widths and heights were estimated from visual inspection

$\%$ The following function will be used to created the simulated spectrum:

$\%$ modelpeaks(WavenumberMSU,NumPeaks, peakshape,Heights,Positions,Widths, 0,0);

$\%$ All required inputs above will be defined and the function will be

$\%$ executed that will return a single output appropiately named 'IntensityMSU'

$\%$ The length of the input matrix represents the Wavenumbers with

$\%$ increaments of 1

$\%$ Further decriptions of the inputs are given in the function's m-file

$\%$ The following lines of code excecute a conditional statement

$\%$ to adjust to the length of the simulated spectrum to the size

$\%$ of the imported scan

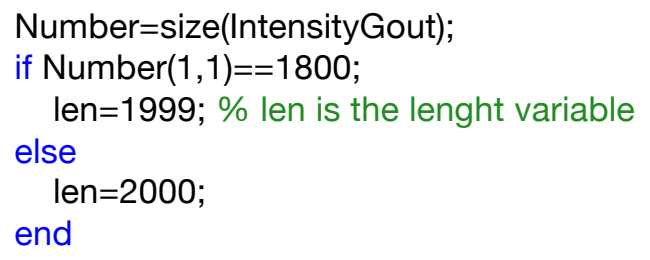

WavenumberMSU=200:round(len); \% defining the length of the MSU standard

NumPeaks=18; \% The number of peaks to model

peakshape $=2$; $\%$ When peakshape input in modelpeaks function is set to 2 ,

$\% \%$ the peaks are created using Lorentzian

$\%$ The heights of the peaks are set

Heights=1*[402060 70 80252530555055205015406815 20];

$\%$ the positions of the peaks represent the location of the MSU peaks

Positions=[386 $40349259163268974978887810141063113012091369 \ldots$ 141914501502 1600];

$\%$ The widths of the peaks at their corresponding postions

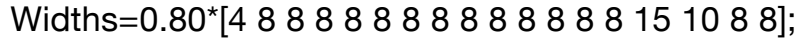


\% The following stores the output of function modelpeaks in 'IntensityMSU' IntensityMSU=modelpeaks(WavenumberMSU,NumPeaks,peakshape,Heights,Positions,... Widths,0,0);

$\% \% \% \% \%$ End of call $2 \% \% \% \% \% \% \%$

$\% \% \% \% \% \% \% \% \% \% \% \% \% \% \% \% \% \%$

$\% \%$ Call \#3: Bacground removal (removal of flouresence)

$\%$ Now the algorithm of background removal will be used

$\%$ Known as Adaptive iterative rewieghted penalized least squares (airPLS)

$\%$ by Z.-M. Zhang, S. Chen, and Y.-Z. Liang, Baseline correction using

$\%$ adaptive iteratively reweighted penalized least squares. Analyst 135 (5),

$\%$ 1138-1146 (2010).

$\%$ https://code.google.com/p/airpls/

$\%$ [AdjustedIntensityGoutPLS,Z]= airPLS(X,lambda,order,wep,p,itermax)

$\%$ where Adjustedlntensity in the corrected intensity and $Z$ is the fitted

$\%$ vector

$\%$ The function will be applied for both Gouty and Healthy patients

$\%$ The required inputs are:

$\% \mathrm{X}$ that is the input matrix, in this case will be the Intensity of their

$\%$ respective scans.

$\%$ Lambda controls the fitting function, larger value, gives a smoother fit

$\%$ The function will determine a baseline fit and subtract the fit from the

$\%$ intensity input.

$\%$ Further description of the inputs are given in the function's m-file

$\%$ airPLS.m

lambda=40; \% baseline fitting parameter for airPLS background removal

$\%$ background removal of Gouty patient:

$\%$ bc stands for background corrected

$\%$ bf stands for background fit

[Gout_bc,Gout_bf]=airPLS(double(IntensityGout'), lambda, 1,0.075,0.1,6);

\%\%\%\% \% -------End of Call 3----------\%\%\%\%\%

\% \% \% \% \% \% \% \% \% \% \% \% \% \% \% \% \% \% \% \%

$\% \%$ Call \# 4: Wavelet denoising function using built-in Matlab function (cmdnoise)

$\%$ Now wavelet denoising will be applied to reduce the noise artifcacts in

$\%$ the scan from call \# 3

$\%$ A built-in function from Matlab named cmdnoise.m will be used to remove noise

$\%$ using wavelet denoising

$\%$ In this function: sigden $=$ cmddenoise(sig,wname,level,sorh)

$\%$ The inputs are sig (The signal from call \#3 to be denoised), level is

$\%$ used for multilevel-1D wavelet decomposition, sorh is the thresholding

$\%$ type, which is set to 's' stands for

$\%$ Soft thresholding, which avoids creating discontinuities while reconstruting.

$\%$ wname is the wavelet used for wavelet analysis.

$\%$ The best option was determined to be bior.3.5.

$\%$ One output is given by the function, sigden which is the denoised signal 
$\%$ This function uses wavlet decompositon and reconstruction with special $\%$ threshold calculation expressions.

$\%$ Increasing level results in a smoother result that somewhat preserves the $\%$ width and shape of the original peaks.

\% The wavelet used for wavelet analysis is 'bior.3.5', this can be changed

Level=2; \% the level of denoising is defined

\% \% \% \% \% \% \% \% \% \% \% \% \% \% \% \% \% \% \% \% \%

$\%$ dn stands for denoised

$\%$ For Gouty patient:

$\%$ Output is stored in Gout_bc_dn

\% \% \% \% \% \% \% \% \% \% \% \% \% \% \% \% \% \% \% \% \%

Gout_bc_dn = cmddenoise(Gout_bc,'bior3.5',Level,'s');

$\%$ Background removal is conduted once more on the denoised signal

$\%$ To provide a more stable normalized result

[Gout_bc_dn,Gout_bf]=airPLS(double(Gout_bc_dn), lambda,1,0.075,0.1,6);

$\% \% \% \% \%$-------End of Call 4-----------\%\%\%\%\%

$\% \% \% \% \% \% \% \% \% \% \% \% \% \% \% \% \% \% \% \% \%$

$\% \%$ Call \# 5 Saving processed scan: processed scan comparision with MSU standard

$\% \% \%$ In this section the processed scan

$\% \% \%$ is plotted along with the MSU standard overlaid

$\% \% \%$ Figure $1 \% \% \%$

$\% \% \%$ the following function removes four character (.spc) from filename

filenamef = filename( 1 :end -4$)$;

$\% \% \%$ Figure is plotted and positioned

plot1=figure('position', [10, 250, 2000, 300]) ;

hold on;

title(filenamef); \% Title for the scan is the filename

plot(WavenumberMSU,IntensityMSU,'m'); \% MSU standard

$\%$ processed scan is also plotted along with its legend and labels

plot(WavenumberGout,Gout_bc_dn, 'k');

legend('Kodati-MSU','Denoised');

xlabel('Wavenumber $\left(\mathrm{cm}^{\wedge}-1\right)$ ');

ylabel('Arbitrary Intensity');

$\%$ The plot is saved in two formats

saveas(plot1, filenamef, 'png')

saveas(plot1, filenamef,'fig')

hold off;

\% \% \% \% \%---------End of Call \# 5---------\%\%\%\%\% 
\% \% \% \% \% \% \% \% \% \% \% \% \% \% \% \% \% \% \% \%

$\% \%$ Call \# 7: Peak detection of Gouty, MSU and Healthy patient

$\%$ built-in function from bioinfomatics toolbox

$\%$ This function will be used to determine the locations of peaks present in

$\%$ scans from Call \# 5

$\%$ Multiple input options are avalible for mspeaks function.

$\%$ The inputs used for this case will be Heightfilter and FWHHfilter.

$\%$ mspeaks(X,Y,'Heightfilter',Heightfiltervalue, 'FWHHFilter',

$\%$ FWHHFiltervalue

$\%$ The value set for Heightfilter will not output peaks below that value

$\%$ of intensity.

$\%$ The value set for FWHHfilter will not output peaks below that value

$\%$ of Full-width at half height.

$\%$ For Gouty patient:

$\%$ Output is stored in peaklist_G

\% The peaks are detected for the processed scan:

[peaklist_G,Fwh]= mspeaks(WavenumberGout,Gout_bc_dn','FWHHFilter' ,2, 'HeightFilter',6);

$\%$ The location of the Peak detected is stored in the following array

Peaklist_loc=floor(peaklist_G(:,1));

$\%$ The Intensity of the Peak detected is stored in the following array

Peaklist_int=(peaklist_G(:,2));

\% Peaks are detected for the standard simulated MSU:

peaklist_S= mspeaks(WavenumberMSU,IntensityMSU,'HeightFilter', 5);

$\% \% \% \% \%$-------End of Call 7--------- $\% \% \% \% \% \%$

$\% \% \% \% \% \% \% \% \% \% \% \% \% \% \% \% \% \% \% \% \% \% \% \%$

$\% \%$ Call \# 9: determining similar peaks

$\%$ In this section A comparision will be conducted between the peaks found

$\%$ in the Gouty patient from call \#7 and MSU standard.

$\%$ The allowed shift from MSU standard will be $+-4 \mathrm{~cm}-1$.

$\%$ First the array containing the peaks of the Gouty patient will be stored

$\%$ in a array called $U$.

$\%$ Then a array of zeroes will be created the size of which will be

$\%$ (size(rows only) of U) x (18)

$\%$ Their are a total of 18 peaks in the MSU standard.

$\%$ The code will run through $U$ (row by row) to check if a value

$\%$ from MSU standard with a shift of +-4 is present in $U$.

$\%$ Finally the dected peaks will be printed on screen.

$\%$ Store Gouty patient peaklist in $U$.

$\%$ The peaklist stored in $U$ can be changed depending on which denoised

$\%$ function is finally chosen. 


\section{U=floor(peaklist_G(:,1));}

ind $=1 ; \%$ ind is initialized to 1 , it will act as a counter for the loop

$\mathrm{sz}=\operatorname{size}(\mathrm{U}) ; \%$ The size of $\mathrm{U}$ is stored in sz

$\%$ a array of zeroes with similar rows to $U$ is created with 18 columns:

$\mathrm{L}=\mathrm{zeros}(\mathrm{sz}(:, 1), 18)$;

\% The following two line will be used to implement the allowable shift:

lowerlimit=round(peaklist_S(:,1)-4);

upperlimit=round(peaklist_S(:,1)+4);

$\%$ The Following loop will will check if the Gouty peaklist array (column one only,

$\%$ has wavenumbers that are present in row (ind) has a value within the range in question

$\%$ for example when the loop begins, the ind value is 1 therefore the range

$\%$ will be between $382<=$ peaklist_Gd(:,1)<=390

$\%$ If there is a value within the range in the Gouty peaklist, then That column

$\% \mathrm{~L}($ :,ind) will have a value of 1 appear once.

for ind $=1: 18$

$\mathrm{L}(:$, ind $)=(($ peaklist_G(:,1)>lowerlimit(ind,:))\& (peaklist_G(:,1)<upperlimit(ind,:)));

end

fprintf('\n\n') \% This line is to improve the asthetic of the list to be displayed

$\%$ The following loop will check every column of array $L$, to see if it has

$\%$ a value of 1 . This will represent that there is a peak present.

$\%$ a column in L coressponds to a row in peaklist_S (MSU standard)

$\% \mathrm{~L}$ has 18 columnn and peaklist_S has 18 rows.

$\%$ For example if a value of one is detected in column one of array $L$, then $\%$ the peak in row one of array peaklist_S was present in the Gouty pateint.

$\%$ the loop will check all columns in array $L$ and display the peak that was

$\%$ present in the Gouty patient.

$\mathrm{k}=1 ; \% \mathrm{~K}$ is initialized to 1 , it will act as a counter for the loop

$\% \% \%$ The following loop will determine and store, whichever ones of the 18 $\% \% \%$ peaks of standard MSU were detected

Peakpresent=zeros $(18,1)$; \% will store the Location of the peak

Peakintensity=zeros $(18,1) ; \%$ will store its corresponding intensity value

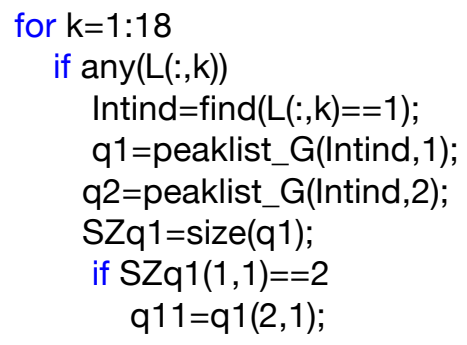




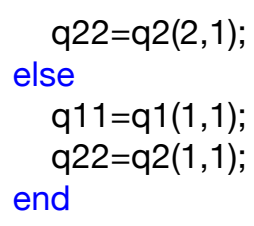

Peakpresent $(k, 1)=q 11$;

Peakintensity $(\mathrm{k}, 1)=\mathrm{q} 22$;

end

end

$\% \% \% \% \%-------$ End of Call 9----------- $\% \% \% \% \%$

$\% \% \% \% \% \% \% \% \% \% \% \% \% \% \% \% \% \% \% \%$

$\% \%$ Call \# 10: Displaying peak analysis results

$\%$ In this section, the total number of peaks found will be calculated and

$\%$ printed on screen.

$\%$ The following loop will check each column of array $L$ to see if there is a

$\%$ value of 1 present. If the value is present then it will store a 1 in the

$\%$ corresponding row in array $\mathrm{Q}$

$\%$ for example if column 3 of $L$ has a value of 1 present (which represents a

$\%$ peak being present), then it will store

$\%$ a value of 1 in row 3 of array $Q$

$\mathrm{j}=1 ; \% \mathrm{j}$ is initialized to 1 , it will act as a counter for the loop

$\mathrm{Q}=$ zeros( $(18,1) ; \%$ a array of zeroes $\mathrm{Q}$ of 18 rows and 1 column

for $\mathrm{j}=1: 18$

$\mathrm{Q}(\mathrm{j},:)=\operatorname{any}(\mathrm{L}(:, \mathrm{j}))$;

end

$\%$ The follwing will sum the number of times the value of 1 appears in array

$\% \mathrm{Q}$. The sum will then be stored in array Total_Peaks_Found

\section{Total_Peaks_Found $=\operatorname{sum}(\mathrm{Q}==1,1)$;}

$\%$ The sum found above will be printed on screen using the following:

fprintf('\n\nThe total number of peaks found were: \%d\n',Total_Peaks_Found)

$\% \% \% \% \% \%$-------End of Call 10---------- $\% \% \% \%$

$\% \% \% \% \% \% \% \% \% \% \% \% \% \% \% \% \% \% \% \% \% \% \%$

$\% \%$ Call \# 11: Exporting results to spreadsheet

Peaktrue=Peakpresent $>0$; \% Converts Peakpresent to binary ( yes or no) of whether MSU peaks were detected

filenameff = cellstr(filenamef); \% converts to string 
\%the following two line create two tables with cariables that are

$\%$ to be exported to a spreadsheet

T1=table(peaklist_S(:,1),Peakpresent,Peakintensity,Peaktrue,Total_Peaks_Found, 'VariableNames',\{'MSU

' 'PeakLoc' 'INT' 'Peakpresent' 'TotalPeaksFound'\});

T2=table(filenameff, 'VariableNames',\{'PatientNo'\});

\%The following code saves the previously created tables

$\%$ in a spreadsheet, for which the name can be changed

$\%$ The sheet number to be saved on is determined from the

$\%$ main program loop counter

$\%$ Thereby each scan will have its own seperate sheet but

$\%$ all the results of the batch processing will be saved in one single

$\%$ spreadsheet

writetable(T1,'Patientsx.xlsx','Sheet',ck);

writetable(T2,'Patientsx.xlsx','Sheet',ck, 'Range','B21:B22');

end $\%$ end of main program loop

clear all

\% \% \% \% \% \% \% \% \% \% \% \% \% \% \% \% \% \% \% \% \% \% \% \% \% \% \%

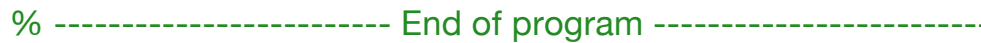

$\% \% \% \% \% \% \% \% \% \% \% \% \% \% \% \% \% \% \% \% \% \% \% \% \% \% \% \% \% \% ~$

\section{Background fluorescence removal function:}

function [AdjustedlntensityPLS, fittedvector]= airPLS(X,lambda,order,wep,p,itermax)

$\%$ Baseline correction using adaptive iteratively reweighted Penalized Least Squares;

$\%$ Input

$\% \quad$ X:row matrix of spectra or chromatogram (size $m^{\star} n, m$ is sample and $n$ is variable)

$\% \quad$ lambda: lambda is an adjustable parameter, it can be adjusted by user. The larger lambda is, the smoother $z$ will be

$\% \quad$ order: an integer indicating the order of the difference of penalties

$\% \quad$ wep: weight exception proportion at both the start and end

$\%$ p: asymmetry parameter for the start and end

$\% \quad$ itermax: maximum iteration times

$\%$ Output

$\% \quad$ Xc: the corrected spectra or chromatogram vector (size $m^{\star} n$ )

$\% \quad$ Z: the fitted vector (size $m^{\star} n$ )

$\%$ Examples:

$\% \quad$ Xc=airPLS(X);

$\% \quad[X c, Z]=a i r P L S(X, 10 e 9,2,0.1,0.5,20)$;

$\%$ Reference:

$\% \quad$ (1) Eilers, P. H. C., A perfect smoother. Analytical Chemistry 75 (14), 3631 (2003).

$\% \quad$ (2) Eilers, P. H. C., Baseline Correction with Asymmetric Least

\% Squares Smoothing, http://www.science.uva.nl/ hboelens/publications/draftpub/Eilers_2005.pdf

$\% \quad$ (3) Gan, Feng, Ruan, Guihua, and Mo, Jinyuan, Baseline correction by improved iterative

polynomial fitting with automatic threshold. Chemometrics and Intelligent Laboratory Systems 82 (1-2), 59 (2006).

$\%$

\% zhimin zhang @ central south university on Mar 30,2011 


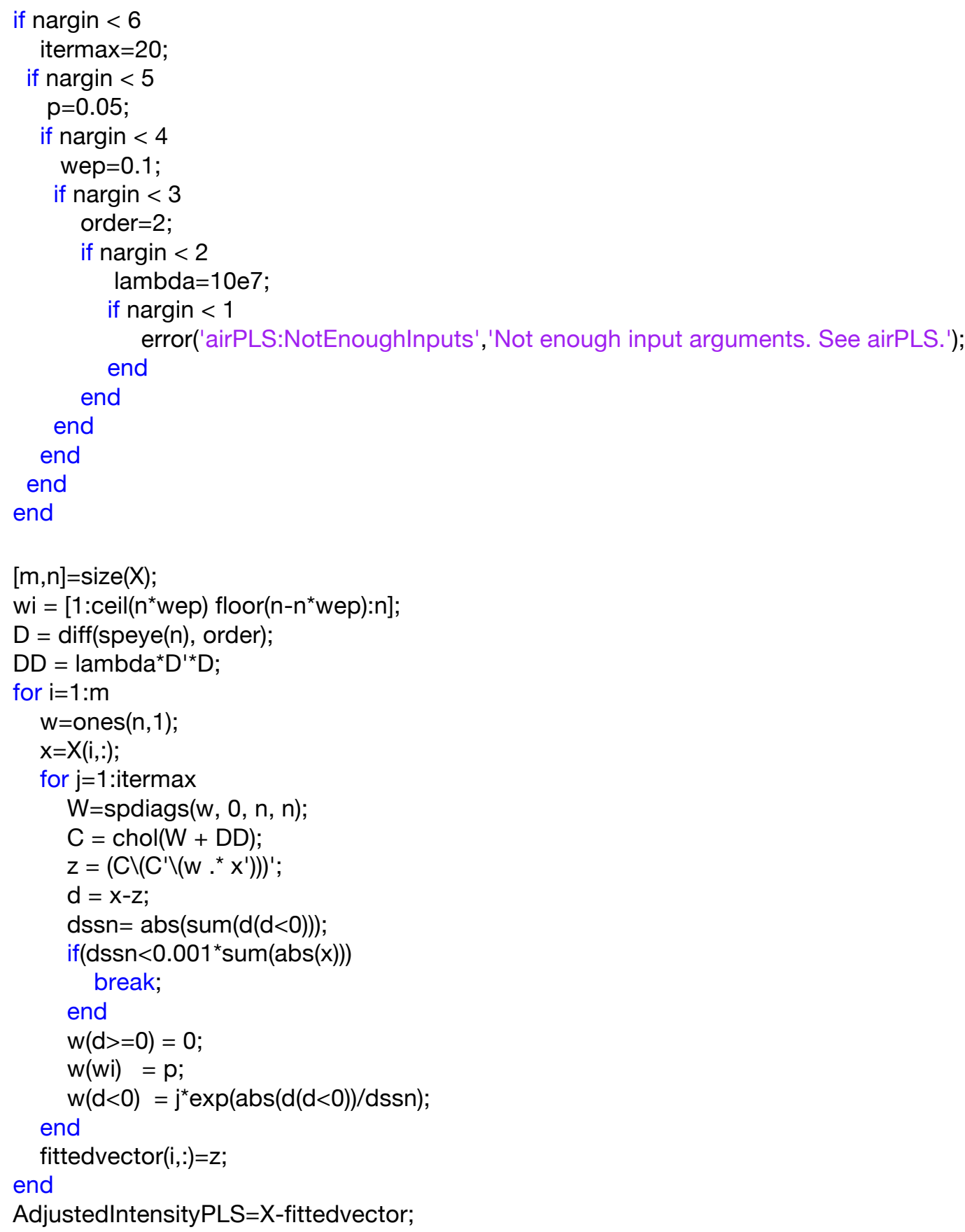




\section{References}


[1] A. Abhishek, D. J. Curran, F. Bilwani, A. C. Jones, M. R. Towler, and M. Doherty, "In vivo detection of monosodium urate crystal deposits by Raman spectroscopy-a pilot study," Rheumatol. (United Kingdom), vol. 55, no. 2, pp. 379-380, 2015.

[2] R. Terkeltaub, Gout: Diagnosis and Management of Gouty Arthritis and Hyperuricemia, 3 edition. Professional Communications, 2013.

[3] H. K. Choi, D. B. Mount, and A. M. Reginato, "Pathogenesis of Gout," Ann. Intern. Med., vol. 143 , no. 7 , p. 499 , Oct. 2005.

[4] B. Li, S. Yang, and O. Akkus, "A customized Raman system for point-of-care detection of arthropathic crystals in the synovial fluid.," Analyst, vol. 139, no. 4, pp. 823-30, 2014.

[5] N. Schlesinger, "Diagnosis of gout: Clinical, laboratory, and radiologic findings," Am. J. Manag. Care, vol. 11, no. SUPPL. 15, pp. 443-450, 2005.

[6] A. J. Luk and P. A. Simkin, "Epidemiology of hyperuricemia and gout," Am. J. Manag. Care, vol. 11, no. SUPPL. 15, pp. 435-442, 2005.

[7] L. Edwards, "Gout," in Primer on the Rheumatic Diseases, 13th Corre., New York, NY: Springer, 2008, pp. 241-262.

[8] M. Fukutomi and K. Kario, "Aging and hypertension.," Expert Rev. Cardiovasc. Ther., vol. 8, no. 11, pp. 1531-1539, 2010.

[9] D. W. Hawkins and D. . Rahn, "Gout and hyperuricemia," Pharmacother. a Pathophsiological Approach, no. Table 1, pp. 1-8, 2005.

[10] R. J. Johnson, D. H. Kang, D. Feig, S. Kivlighn, J. Kanellis, S. Watanabe, K. R. Tuttle, B. Rodriguez-Iturbe, J. Herrera-Acosta, and M. Mazzali, "Is there a pathogenetic role for uric acid in hypertension and cardiovascular and renal disease?," Hypertension, vol. 41, no. 6, pp. 1183-1190, 2003.

[11] E. W. Campion, R. J. Glynn, and L. O. Delabry, "Asymptomatic hyperuricemia. Risks and consequences in the normative aging study," Am. J. Med., vol. 82, no. 3, pp. 421-426, Mar. 1987.

[12] K. C. Lin, H. Y. Lin, and P. Chou, "The interaction between uric acid level and other risk factors on the development of gout among asymptomatic hyperuricemic men in a prospective study.," J. Rheumatol., vol. 27, no. 6, pp. 1501-5, Jun. 2000.

[13] J. N. Loeb, "The influence of temperature on the solubility of monosodium urate," Arthritis Rheum., vol. 15, no. 2, pp. 189-192, 1972. 
[14] S. Stewart, N. Dalbeth, A. C. Vandal, and K. Rome, "The first metatarsophalangeal joint in gout: a systematic review and meta-analysis," BMC Musculoskelet. Disord., 2016.

[15] N. Schlesinger, "Management of acute and chronic gouty arthritis: present state-of-theart.," Drugs, vol. 64, no. 21, pp. 2399-416, 2004.

[16] K. Y. Kim, H. Ralph Schumacher, E. Hunsche, A. I. Wertheimer, and S. X. Kong, “A literature review of the epidemiology and treatment of acute gout," Clin Ther, vol. 25, pp. 1593-1617, 2003.

[17] R. Terkeltaub, "Emerging uricosurics for gout," Expert Rev. Clin. Pharmacol., vol. 0, no. 0, pp. 1-3, 2016.

[18] M. Doherty, "New insights into the epidemiology of gout," Rheumatology, vol. 49, no. 3, pp. 613-614, 2009.

[19] Y. Zhu, B. J. Pandya, and H. K. Choi, "Prevalence of gout and hyperuricemia in the US general population: The National Health and Nutrition Examination Survey 2007-2008," Arthritis Rheum., vol. 63, no. 10, pp. 3136-3141, 2011.

[20] C.-F. Kuo, M. J. Grainge, W. Zhang, and M. Doherty, "Global epidemiology of gout: prevalence, incidence, and risk factors," Nat. Rev. Rheumatol., vol. 11, no. 11, pp. 649$662,2015$.

[21] T. R. Mikuls, J. T. Farrar, W. B. Bilker, S. Fernandes, H. R. Schumacher, and K. G. Saag, “Gout epidemiology: results from the UK General Practice Research Database, 19901999.," Ann. Rheum. Dis., vol. 64, no. 2, pp. 267-72, 2005.

[22] L. Annemans, E. Spaepen, M. Gaskin, M. Bonnemaire, V. Malier, T. Gilbert, and G. Nuki, "Gout in the UK and Germany: prevalence, comorbidities and management in general practice 2000-2005.," Ann. Rheum. Dis., vol. 67, no. 7, pp. 960-6, 2008.

[23] J. Zalokar, J. Lellouch, J. R. Claude, and D. Kuntz, "Serum uric acid in 23,923 men and gout in a subsample of 4257 men in France," J. Chronic Dis., vol. 25, no. 5, pp. 305-312, 1972.

[24] E. Roddy and M. Doherty, "Epidemiology of gout.," vol. 75 Suppl 5, no. July, pp. S9-12, 2008.

[25] S. W. Graf, R. Buchbinder, J. Zochling, and S. L. Whittle, "The accuracy of methods for urate crystal detection in synovial fluid and the effect of sample handling: A systematic review," Clin. Rheumatol., vol. 32, no. 2, pp. 225-232, 2013. 
[26] P. Courtney and M. Doherty, "Joint aspiration and injection and synovial fluid analysis," Best Pract. Res. Clin. Rheumatol., vol. 27, no. 2, pp. 137-169, 2013.

[27] R. Von Essen and a M. Hölttä, "Quality control of the laboratory diagnosis of gout by synovial fluid microscopy.," Scand. J. Rheumatol., vol. 19, no. 3, pp. 232-4, 1990.

[28] R. von Essen, a M. Hölttä, and R. Pikkarainen, “Quality control of synovial fluid crystal identification.," Ann. Rheum. Dis., vol. 57, no. 2, pp. 107-109, 1998.

[29] P. Hasselbacher, "Variation in synovial fluid analysis by hospital laboratories," Arthritis Rheum., vol. 30, no. 6, pp. 637-642, 1987.

[30] J. W. Park, D. J. Ko, J. J. Yoo, S. H. Chang, H. J. Cho, E. H. Kang, J. K. Park, Y. W. Song, and Y. J. Lee, "Clinical factors and treatment outcomes associated with failure in the detection of urate crystal in patients with acute gouty arthritis.," Korean J. Intern. Med., vol. 29, no. 3, pp. 361-9, 2014.

[31] C. Xingguo, G. H. Donard, H. Y. Russel, N. Y. Yener, and A. Ozan, “Analysis of crystals leading to joint arthropathies by raman spectroscopy: Comparison with compensated polarized imaging," Appl. Spectrosc., vol. 63, no. 4, pp. 381-386, 2009.

[32] L. X. Chen and H. R. Schumacher, "Gout: can we create an evidence-based systematic approach to diagnosis and management?," Best Pract. Res. Clin. Rheumatol., vol. 20, no. 4, pp. 673-684, 2006.

[33] R. G. Thiele, "Diagnosis of gout by ultrasound," Rheumatology, vol. 46, no. 7, pp. 11161121, Jul. 2007.

[34] N. Dalbeth and A. J. Doyle, "Imaging of gout - An overview," Best Pract. Res. Clin. Rheumatol., vol. 26, no. 6, pp. 823-838, Dec. 2012.

[35] T. Rettenbacher, S. Ennemoser, H. Weirich, H. Ulmer, F. Hartig, W. Klotz, and M. Herold, "Diagnostic imaging of gout: Comparison of high-resolution US versus conventional X-ray,” Eur. Radiol., vol. 18, no. 3, pp. 621-630, 2008.

[36] F. M. Mcqueen, A. Doyle, and N. Dalbeth, "Imaging in gout - What can we learn from MRI , CT , DECT and US ?,” 2011.

[37] G. Girish, K. N. Glazebrook, and J. A. Jacobson, “Advanced imaging in gout,” Am. J. Roentgenol., vol. 201, no. 3, pp. 515-525, 2013.

[38] A. N. Baer, T. Kurano, U. J. Thakur, G. K. Thawait, M. K. Fuld, J. W. Maynard, M. McAdams-DeMarco, E. K. Fishman, and J. A. Carrino, "Dual-energy computed 
tomography has limited sensitivity for non-tophaceous gout: a comparison study with tophaceous gout," BMC Musculoskelet. Disord., vol. 17, no. 1, p. 91, 2016.

[39] H. K. Choi, a M. Al-Arfaj, a Eftekhari, P. L. Munk, K. Shojania, G. Reid, and S. Nicolaou, "Dual energy computed tomography in tophaceous gout.," Ann. Rheum. Dis., vol. 68, no. 10, pp. 1609-1612, 2009.

[40] I. Watt and H. Middlemiss, "The radiology of gout," Clin. Radiol., vol. 26, pp. 27-36, Jan. 1975.

[41] F. Perez-Ruiz, N. Dalbeth, A. Urresola, E. de Miguel, and N. Schlesinger, "Imaging of gout: findings and utility.," Arthritis Res. Ther., vol. 11, no. 3, p. 232, 2009.

[42] C. Kallaway, L. M. Almond, H. Barr, J. Wood, J. Hutchings, C. Kendall, and N. Stone, "Advances in the clinical application of Raman spectroscopy for cancer diagnostics," Photodiagnosis Photodyn. Ther., vol. 10, no. 3, pp. 207-219, Sep. 2013.

[43] V. R. Kodati, A. T. Tu, and J. L. Turumin, "Raman spectroscopic identification of uricacid-type kidney stone," Appl. Spectrosc., vol. 44, no. 7, pp. 1134-1136, 1990.

[44] W. E. Smith and G. Dent, Modern Raman Spectroscopy - A pratical Approach. John Wiley \& Sons , Ltd, 2005.

[45] A. T. Tu, Raman Spectroscopy in Biology: Principles and Applications. Wiley, 1982.

[46] J. R. Ferraro, K. Nakamoto, and C. W. Brown, Introductory Raman Spectroscopy. 2003.

[47] D. A. Long, "The Raman Effect:A Unified Treatment of the Theory of Raman Scattering by Molecules.," John Wiley Sons Ltd, vol. 8, p. 611, 2002.

[48] C. V. RAMAN and K. S. KRISHNAN, "A New Type of Secondary Radiation,” Nature, vol. 121 , no. 3048, pp. 501-502, 1928.

[49] -Ing Bernhard Schrader, Ed., "Infrared and Raman Spectroscopy," in Zeolite Chemistry and Catalysis, Wiley-VCH Verlag GmbH, 2009, pp. 197-222.

[50] Q. Tu and C. Chang, "Diagnostic applications of Raman spectroscopy," Nanomedicine Nanotechnology, Biol. Med., vol. 8, no. 5, pp. 545-558, 2012.

[51] P. J. Larkin, "IR and Raman Spectroscopy - Principles and Spectral Interpretation." Elsevier, 2011.

[52] P. Matousek and N. Stone, "Recent advances in the development of Raman spectroscopy for deep non-invasive medical diagnosis," J. Biophotonics, vol. 6, no. 1, pp. 7-19, 2013.

[53] M. V. Schulmerich, J. H. Cole, J. M. Kreider, F. Esmonde-White, K. A. Dooley, S. A. 
Goldstein, and M. D. Morris, “Transcutaneous Raman Spectroscopy of Murine Bone $<\mathrm{I}>$ In Vivo</I>," Appl. Spectrosc., vol. 63, no. 3, pp. 286-295, 2009.

[54] S. Naito, Y.-K. Min, K. Sugata, O. Osanai, T. Kitahara, H. Hiruma, and H. Hamaguchi, "In vivo measurement of human dermis by 1064 nm-excited fiber Raman spectroscopy.," Skin Res. Technol., vol. 14, no. 1, pp. 18-25, Feb. 2008.

[55] E. E. Lawson, H. G. M. Edwards, A. C. Williams, and B. W. Barry, "Applications of Raman spectroscopy to skin research," Skin Research and Technology, vol. 3, no. 3. pp. 147-153, Aug-1997.

[56] C. Kendall, M. Isabelle, F. Bazant-Hegemark, J. Hutchings, L. Orr, J. Babrah, R. Baker, and N. Stone, "Vibrational spectroscopy: a clinical tool for cancer diagnostics," Analyst, vol. 134, no. 6, pp. 1029-1045, 2009.

[57] A. Synytsya, M. Judexova, D. Hoskovec, M. Miskovicova, and L. Petruzelka, "Raman spectroscopy at different excitation wavelengths (1064, 785 and 532nm) as a tool for diagnosis of colon cancer," J. Raman Spectrosc., vol. 45, no. 10, pp. 903-911, 2014.

[58] Y. K. Min, T. Yamamoto, E. Kohda, T. Ito, and H. O. Hamaguchi, "1064 nm nearinfrared multichannel Raman spectroscopy of fresh human lung tissues," J. Raman Spectrosc., vol. 36, no. 1, pp. 73-76, 2005.

[59] J. Zhao, H. Lui, D. I. McLean, and H. Zeng, "Real-time Raman spectroscopy for noninvasive skin cancer detection - preliminary results.," Conf. Proc. IEEE Eng. Med. Biol. Soc., vol. 2008, pp. 3107-3109, 2008.

[60] C. A. Lieber, S. K. Majumder, D. L. Ellis, D. D. Billheimer, and A. Mahadevan-Jansen, "In vivo nonmelanoma skin cancer diagnosis using Raman microspectroscopy," Lasers Surg. Med., vol. 40, no. 7, pp. 461-467, 2008.

[61] A. A. Bunaciu, H. Y. Aboul-Enein, and Ş. Fleschin, "Vibrational spectroscopy in clinical analysis," Appl. Spectrosc. Rev., vol. 50, no. 2, pp. 176-191, 2015.

[62] W. Wang, J. Zhao, M. Short, and H. Zeng, Real-time in vivo cancer diagnosis using raman spectroscopy. 2014.

[63] J. C. C. Day, R. Bennett, B. Smith, C. Kendall, J. Hutchings, G. M. Meaden, C. Born, S. Yu, and N. Stone, “A miniature confocal Raman probe for endoscopic use," Phys. Med. Biol., vol. 54, no. 23, pp. 7077-7087, Dec. 2009.

[64] M. A. Short, S. Lam, A. M. McWilliams, D. N. Ionescu, and H. Zeng, "Using laser raman 
spectroscopy to reduce false positives of autofluorescence bronchoscopies: A pilot study," J. Thorac. Oncol., vol. 6, no. 7, pp. 1206-1214, 2011.

[65] J. Shao, M. Lin, Y. Li, X. Li, J. Liu, J. Liang, and H. Yao, "In Vivo Blood Glucose Quantification Using Raman Spectroscopy,” PLoS One, vol. 7, no. 10, 2012.

[66] C. C. Pelletier, J. L. Lambert, and M. Borchert, "Determination of glucose in human aqueous humor using Raman spectroscopy and designed-solution calibration," Appl. Spectrosc., vol. 59, no. 8, pp. 1024-1031, 2005.

[67] J. Sebag, S. Nie, K. Reiser, M. A. Charles, and N. T. Yu, "Raman spectroscopy of human vitreous in proliferative diabetic retinopathy," Invest Ophthalmol Vis Sci, vol. 35, no. 7, pp. 2976-2980, 1994.

[68] K. A. Esmonde-White, F. W. L. Esmonde-White, C. M. Holmes, M. D. Morris, and B. J. Roessler, "Alterations to bone mineral composition as an early indication of osteomyelitis in the diabetic foot," Diabetes Care, vol. 36, no. 11, pp. 3652-3654, 2013.

[69] R. Smith and I. U. Rehman, "Fourier transform Raman spectroscopic studies of human bone,” J. Mater. Sci. Mater. Med., vol. 5, pp. 775-778, 1994.

[70] J. W. Ager, R. K. Nalla, K. L. Breeden, and R. O. Ritchie, "Deep-ultraviolet Raman spectroscopy study of the effect of aging on human cortical bone.," J. Biomed. Opt., vol. 10, no. 3, p. 34012, 2005.

[71] M. D. Morris and G. S. Mandair, "Raman assessment of bone quality," in Clinical Orthopaedics and Related Research, 2011, vol. 469, no. 8, pp. 2160-2169.

[72] Z. Sun, A. Goldhirsch, K. N. Price, M. Colleoni, A. Ravaioli, E. Simoncini, I. Campbell, R. D. Gelber, and M. Towler, "Bone Quality Test (BQT) scores of fingernails in postmenopausal patients treated with adjuvant letrozole or tamoxifen for early breast cancer," Breast, vol. 18, no. 2, pp. 84-88, Apr. 2009.

[73] N. M. Cummins, J. C. C. Day, A. Wren, P. Carroll, N. Murphy, P. M. Jakeman, and M. R. Towler, "Raman spectroscopy of fingernails: A novel tool for evaluation of bone quality?," Spectroscopy, vol. 24, no. 5, pp. 517-524, 2010.

[74] P. Moran, M. R. Towler, S. Chowdhury, J. Saunders, M. J. German, N. S. Lawson, H. M. Pollock, I. Pillay, and D. Lyons, "Preliminary work on the development of a novel detection method for osteoporosis," J. Mater. Sci. Mater. Med., vol. 18, no. 6, pp. 969974, Jun. 2007. 
[75] I. Pillay, D. Lyons, M. J. German, N. S. Lawson, H. M. Pollock, J. Saunders, S. Chowdhury, P. Moran, and M. R. Towler, "The use of fingernails as a means of assessing bone health: a pilot study.," J. Womens. Health (Larchmt)., vol. 14, no. 4, pp. 339-344, May 2005.

[76] J. R. Beattie, N. M. Cummins, C. Caraher, O. M. O. Driscoll, A. T. Bansal, R. Eastell, S. H. Ralston, M. D. Stone, G. Pearson, and M. R. Towler, "Raman Spectroscopic Analysis of Fingernail Clippings Can Help Differentiate Between Postmenopausal Women Who Have and Have Not Suffered a Fracture," pp. 109-116, 2016.

[77] P. J. Caspers, G. W. Lucassen, R. Wolthuis, H. A. Bruining, and G. J. Puppels, "In vitro andin vivo Raman spectroscopy of human skin," Biospectroscopy, vol. 4, no. S5, pp. S31S39, 1998.

[78] Z. Movasaghi, S. Rehman, and I. Rehman, "Raman spectroscopy of biological tissues," Appl. Spectrosc. ..., vol. 42, no. 5, pp. 493-541, 2007.

[79] S. Yang, B. Li, M. N. Slipchenko, A. Akkus, N. G. Singer, Y. N. Yeni, and O. Akkus, "Laser wavelength dependence of background fluorescence in Raman spectroscopic analysis of synovial fluid from symptomatic joints," J. Raman Spectrosc., vol. 44, no. 8, pp. 1089-1095, 2013.

[80] D. J. Curran, L. Rubin, and M. R. Towler, "Raman Spectroscopy Applied to the Noninvasive Detection of Monosodium Urate Crystal Deposits," Clin Med Insights Arthritis Musculoskelet Disord., vol. 8, pp. 55-58, 2015.

[81] I. Peláez-Ballestas, C. Hernández Cuevas, R. Burgos-Vargas, L. Hernández Roque, L. Terán, J. Espinoza, J. A. Esquivel-Valerio, M. V. Goycochea-Robles, F. J. Aceves, A. G. Bernard, L. Ventura, C. Shumsky, A. Hernández Garduño, and J. Vázquez-Mellado, "Diagnosis of chronic gout: evaluating the american college of rheumatology proposal, European league against rheumatism recommendations, and clinical judgment.," $J$. Rheumatol., vol. 37, no. 8, pp. 1743-8, Aug. 2010.

[82] Z.-M. Zhang, S. Chen, and Y.-Z. Liang, "Baseline correction using adaptive iteratively reweighted penalized least squares.," Analyst, vol. 135, no. 5, pp. 1138-46, May 2010.

[83] E. Roddy, W. Zhang, M. Doherty, and E. Roddy, “Are joints affected by gout also affected by osteoarthritis ?," Ann. Rheum. Dis., vol. 66, no. 10, pp. 1374-1378, 2007.

[84] E. Roddy and M. Doherty, "Gout and osteoarthritis: A pathogenetic link?," Jt. Bone Spine, 
vol. 79 , no. 5, pp. 425-427, 2012.

[85] E. Pascual and F. Sivera, "Time required for disappearance of urate crystals from synovial fluid after successful hypouricaemic treatment relates to the duration of gout.," Ann. Rheum. Dis., vol. 66, no. 8, pp. 1056-8, 2007.

[86] N. Dalbeth and A. J. Doyle, "Imaging of gout: an overview.," Best Pract. Res. Clin. Rheumatol., vol. 26, no. 6, pp. 823-38, 2012.

[87] A. Swan, H. Amer, and P. Dieppe, "The value of synovial fluid assays in the diagnosis of joint disease: a literature survey.," Ann. Rheum. Dis., vol. 61, no. 6, pp. 493-8, 2002. 